\title{
Single and Double Change Covering Designs
}

\author{
Amanda Chafee
}

2021 


\title{
Single and Double Change Covering Designs
}

\author{
by \\ Amanda Chafee
}

\author{
A thesis submitted to \\ the Faculty of Graduate Studies and Research \\ in partial fulfillment of the requirements for the degree of \\ Master of Science \\ in \\ Mathematics with Concentration in Mathematics \\ Carleton University \\ Ottawa, Ontario, Canada \\ April, 2021 \\ (c) Copyright 2021 \\ Amanda Chafee
}




\section{Abstract}

A single change covering design (SCCD) is a sequence of $b k$-sets, called blocks, of a $V$-set in which exactly one element differs between consecutive blocks and every $s$-set of $V$ is in some block.

We will review the literature and discuss several recursive constructions which completely solve the existence of SCCD for $k=3,4$ and partially for $k=5$. We will examine optimizations on exhaustive search techniques. We determined that there are 313 unique circular $\operatorname{SCCD}(12,4,2,22)$. We determine a recursion for $s=3$ and general $k$ using expansion sets.

A double change covering design (DCCD) is similarly defined but consecutive blocks differ by two elements. We will completely solve the existence of tight DCCD $k=3, s=2$. We give several constructions using recursion and algebraic difference methods. This provides us with constructions for circular $\operatorname{DCCD}(4 k-2,2 k, 2,2 k-1)$, circular $\operatorname{DCCD}(4 k-1,2 k+1,2,2 k-1)$ and circular $\operatorname{DCCD}(4 k-5 k, 2,4 k-2)$ exists. We also find some other circular DCCD with given $v$ and $k$. 


\section{Acknowledgements}

I would first like to thank my supervisor, Brett Stevens, for providing guidance, feedback and support though out my Master's degree. I am so grateful that when I started this journey in my undergraduate years he introduced me to single change covering designs. I am also very thankful that we started this journey pre-Covid-19 and that video conferencing allowed us to continue meeting virtually. I would also like to thank my committee members Steven Wang, Michael Newman, and Root Gorelick and chair, Saban Alaca, for their time.

Heartfelt thanks to my parents, siblings and relatives for supporting me through this academic journey and encouraging me to pursue what I love. Also, thanks for letting me take over the games table at the cottage all last summer. I am truly blessed to have such an amazing family.

Thank you to all the old and new friends I have made during this process. They were all so encouraging, supportive and wonderful. Thank you all, I could not have done this without you.

A big thank you to my roommates who put up with my shenanigans while on this journey.

This work was partially supported by Mitacs though the Mitacs RTA program. 


\section{Contents}

$\begin{array}{lll}\text { Abstract } & \text { ii }\end{array}$

Acknowledgements

Contents

List of Tables $\quad$ v

List of Figures vii

1 Preliminaries 1

1.1 Introduction . . . . . . . . . . . . . . . . 1

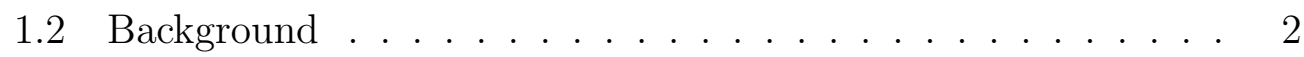

1.3 Literature Review . . . . . . . . . . . . . . 5

2 Single Change Covering Designs 25

2.1 Efficient Computational Search

Implementation . . . . . . . . . . . . . . . . 27

2.2 Algorithms . . . . . . . . . . . . . . . . . . . . . 29

2.2.1 SF-arrays . . . . . . . . . . . . . . . 34

2.3 Higher Strength SCCD . . . . . . . . . . . . . . 35

3 Double Change Covering Designs 39

3.1 Difference Methodology . . . . . . . . . . . . . . . 43

4 Conclusion $\quad \mathbf{5 2}$

4.1 Looking forward . . . . . . . . . . . . . . 53

$\begin{array}{ll}\text { Bibliography } & 61\end{array}$ 


\section{List of Tables}

1.1 STS(7) constructed from the Fano Plane [13] . . . . . . . . 3

1.2 Using a STS(7) to test pairwise interactions. [13] . . . . . . . 4

$1.3(9,3,1)$-BIBD $[13] \ldots \ldots \ldots \ldots$

$1.4 \quad(13,3,1)-B I B D \ldots \ldots \ldots \ldots \ldots$

$1.5 \operatorname{SCCD}(7,3,2,10) \ldots \ldots \ldots \ldots \ldots \ldots$

1.6 $\operatorname{CSCCD}(6,3,2,8)$. The pair $\{1,4\}$ is covered in $B_{5}$ and $B_{8}$. [8] . 6

1.7 economical $\operatorname{SCCD}(4,3,2,3) \ldots \ldots \ldots \ldots$

1.8 Tight single change blocks . . . . . . . . . . . . . . . . 12

1.9 economical $\operatorname{SCCD}(8,3,2,14) \quad \ldots \ldots \ldots$

$1.10 \operatorname{SCCD}(12,4,2,21)[10] \ldots \ldots \ldots \ldots$

1.11 tight $\operatorname{CSCCD}(8,3,2,14) \ldots \ldots \ldots \ldots$

1.12 SF-arrays for tight $\operatorname{CSCCD}(8,3,2,14) \ldots \ldots . . \ldots 16$

1.13 SF-arrays for the tight $\operatorname{CSCCD}(9,4,2,12)$. . . . . . . . . . 18

1.14 skeleton for the $\operatorname{SF}$-array (a) of a tight $\operatorname{CSCCD}(9,4,2,12)$. . 18

1.15 Partially filled in tight $\operatorname{CSCCD}(9,4,2,12) \ldots \ldots$

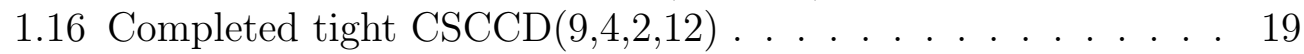

1.17 Tight $\operatorname{SCCD}(10,3,2,22)[12]$ with a disjoint-capable expansion set. . . . . . . . . . . . . . 20

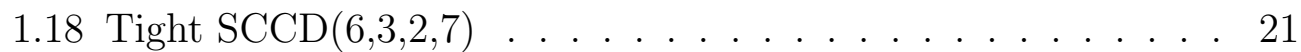

1.19 Tight $\operatorname{CSCCD}(12,3,2,33) \quad \ldots \ldots \ldots . \ldots . \ldots . \ldots 21$

2.1 Consecutive blocks to replace . . . . . . . . . . . . 25

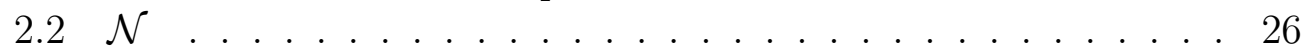

2.3 Tight $\operatorname{SCCD}(6,3,2,7) \ldots \ldots \ldots \ldots$

2.4 The extended tight $\operatorname{SCCD}(6,3,2,7)$ with the 14 inserted blocks 27

2.5 The completed tight $\operatorname{SCCD}(10,3,2,22)$ based on the tight $\operatorname{SCCD}(6,3,2,7)$ 27 
3.1 A tight $\operatorname{CDCCD}(7,3,2,7) \ldots \ldots . \ldots . \ldots . . \ldots 41$

3.2 A DCCD $(15,3,2,35)$ built from the $\operatorname{DCCD}(7,3,2,2)$ and $K_{8}$. . 43

3.3 A tight $\operatorname{CSCCD}(3,2,2,3)$ and tight $\operatorname{CSCCD}(6,4,2,3) \quad \ldots . . .44$

3.4 A tight $\operatorname{CDCCD}(10,4,2,9) \ldots \ldots \ldots . \ldots . \ldots . \ldots 44$

$3.5 \mathrm{~A} \operatorname{CDCCD}(11,4,2,11) \ldots \ldots \ldots . \ldots . \ldots 46$

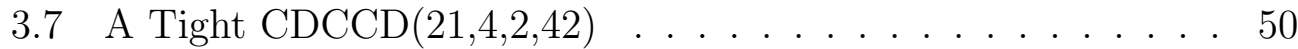

3.8 Tight $\operatorname{CDCCD}(13,3,2,26) \ldots \ldots \ldots \ldots . \ldots \ldots$

4.1 Partial example of $k n$ people in $n$ groups of $k, t=2 \ldots$. . . 54 


\section{List of Figures}

1.1 1-factor of $K_{6} \ldots \ldots \ldots \ldots \ldots$

1.2 Fano Plane . . . . . . . . . . . . . . . . . . 3

1.3 The block intersection graph of $\operatorname{STS}(7) \ldots \ldots . \ldots 5$

$3.1 \mathrm{~K}_{8} \ldots \ldots \ldots \ldots \ldots \ldots$

$3.2 c=1, k=4$ difference construction for $\operatorname{DCCD}(11,4,2,11)$. . 45

$3.3 c=2, k=3$ tight $\operatorname{CDCCD}(13,3,2,26) \ldots \ldots \ldots . \ldots . . \ldots 51$ 


\section{Chapter 1}

\section{Preliminaries}

\subsection{Introduction}

A single change covering design (SCCD) is a sequence of $b k$-sets, called blocks, of a $V$-set in which exactly one element differs between consecutive blocks and every $s$-set of $V$ is in some block. We notate this $\operatorname{SCCD}(v, k, s, b)$.

In this chapter we begin by reviewing relevant background information in design theory, graph theory and algebraic difference that will be needed in this theory. Continuing, we review all previous research on single change covering designs. Significant contributions were made to single change covering design theory during a twelve year period between 1990 and 2001 which will be discussed in Chapter 1.3. We conclude Chapter 1 by summarizing the known designs with $k=2, \ldots, 5$.

In Chapter 2 we will discuss progress made to SCCD theory during the course of this thesis. We have a new recursive construction for strength 2 SCCD and CSCCD. We examine and combine previous exhaustive search techniques and implement an exhaustive search for circular SCCD. Using this we find all 313 possible non-equivalent $\operatorname{CSCCD}(12,4,2,21)$. We conclude this chapter by exploring preliminary results for strength 3 CSCCD with the goal of extending this search for higher strength designs.

We generalize the size of a change in Chapter 3 and focus double change covering designs. We will completely solve the existence question for tight DCCD and tight CDCCD when $k=2, s=2$. We prove some recursive constructions analogous to those of SCCD. We then find CDCCD by using algebraic difference methods. We conclude the chapter by combining some 
recursions and stating some sporadic results.

Finally, we will review the progress made in this thesis and consider future research paths that may be taken. Consequently we define some abstracted change designs for consideration.

\section{$1.2 \quad$ Background}

A graph is an ordered pair $G=(V, E)$ of vertices, $V$, and edges, $E$, such that each edge consists of two end vertices. [16]

A walk in a graph $G$ is a sequence $W=v_{0} e_{1} v_{1} \ldots v_{l-1} e_{l} v_{l}$ where $v$ and $e$ are alternating vertices and edges of $G$ such that $v_{i-1}$ and $v_{i}$ are the ends of edge $e_{i}, 1 \leq i \leq l$.

A path in a graph $G$ is a walk with distinct vertices.

A cycle in a graph $G$ is a path in $G$ where the first and last vertex are joined by an edge.

A Hamilton path (Hamilton cycle) in a graph $G$ is a path (cycle) visiting all the vertices $V$ of $G$ only once.

A perfect matching or 1-factor of a graph $G$ is a subset of edges $M$ such that every vertex $v \in V$ is adjacent to exactly one edge $e \in M$. A partition of the edges of a graph into perfect matchings is a 1-factorization. Each color in Figure 1.3 is a 1-factor of the 1-factorization of $K_{6}$.

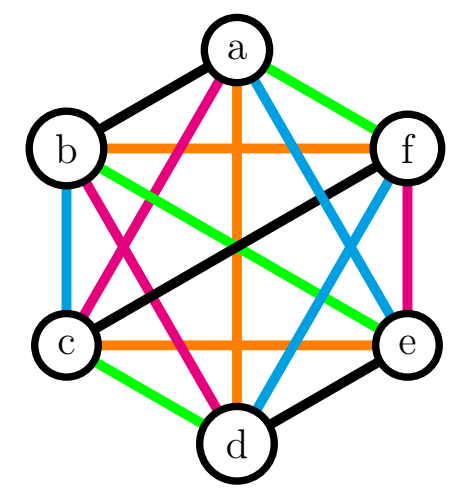

Figure 1.1: 1-factor of $K_{6}$

A Steiner system $\mathrm{S}(t, k, v), 2 \leq t \leq k \leq v$, is a $v$-set $V$ with a family $\mathcal{B}$ of $k$-subsets of $V$ (blocks), such that every $t$-subset of $V$ is contained in 
exactly one block, $t, k, v \in \mathbb{Z}^{+}$. [4]

A Steiner Triple System, STS $(v)$, is a $\mathrm{S}(2,3, v)$. A STS(7) is shown in Figure 1.2 and it's blocks are listed in Table 1.1. A Steiner Quadruple System, $\operatorname{SQS}(v)$, is a $\mathrm{S}(3,4, v)$.

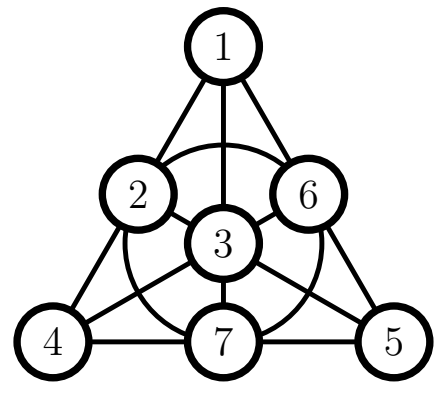

Figure 1.2: Fano Plane

$\begin{array}{lllllll}\mathrm{B}_{1} & \mathrm{~B}_{2} & \mathrm{~B}_{3} & \mathrm{~B}_{4} & \mathrm{~B}_{5} & \mathrm{~B}_{6} & \mathrm{~B}_{7} \\ 1 & 2 & 3 & 4 & 5 & 6 & 7 \\ 2 & 3 & 4 & 5 & 6 & 7 & 1 \\ 4 & 5 & 6 & 7 & 1 & 2 & 3\end{array}$

Table 1.1: STS(7) constructed from the Fano Plane [13]

Fisher studied the applications of combinatorial designs to experimental design in his 1935 book "The Design of Experiments" [5]. In "The Design of Experiments" Fisher examines agricultural experiments where a scheme is used to plant wheat in fields and analyse the crop yield to improve farming techniques. We think of each field as a block with the space to grow $k$ varieties of wheat. Today, designs are used for many diverse applications [2, $17,4]$.

The designs Fisher implemented in his experiment were balanced incomplete block designs $((v, k, \lambda)$-BIBD), which is a set of blocks from a $v$-set $X$ where each pair from $X$ occurs in exactly $\lambda$ blocks. $\operatorname{An} \operatorname{STS}(v)$ is a $(v, 3,1)$-BIBD.

Let $(G,+, 0)$ be a finite group of order $v$. For $D \subseteq G$ let $\partial D=\left\{d_{1}-d_{2}\right.$ : $\left.d_{1} \neq d_{2} \in D\right\}$.

A $(v, k, \lambda)$-difference family is a collection $\left\{D_{1}, \ldots, D_{t}\right\}$ of $k$-subsets of $G,|G|=v$, where $\partial D_{1} \cup \ldots \cup \partial D_{t}=\lambda G \backslash\{0\}$. A difference family with $t=1$ is a $(v, k, \lambda)$-difference set. 
Example 1.1. $D=\{1,3,4\}$ is a $(7,3,1)$-difference set in $\mathbb{Z}_{7}$.

Example 1.2. $\{\{0,1,4\},\{0,2,8\}\}$ is a $(13,3,1)$-difference family over $\mathbb{Z}_{13}$.

Suppose an electronics company has seven versions of a component that it wants to test interacting over a network. They can only test three at a time. The STS(7) offers a solution that tests the interactions between all pairs with seven tests. Table 1.2 gives a set of tests derived this way.

$\begin{array}{lccccccc} & \text { Test 1 } & \text { Test 2 } & \text { Test 3 } & \text { Test } 4 & \text { Test } 5 & \text { Test } 6 & \text { Test } 7 \\ \text { Component 1: } & 1 & 1 & 1 & 2 & 2 & 3 & 3 \\ \text { Component 2: } & 2 & 4 & 6 & 4 & 5 & 4 & 5 \\ \text { Component 3: } & 3 & 5 & 7 & 6 & 7 & 7 & 6\end{array}$

Table 1.2: Using a $\operatorname{STS}(7)$ to test pairwise interactions. [13]

As the company increases the number of components they will need many more blocks to test them. For example, twelve tests are required if there are nine components, while twenty-six tests are required if there are thirteen components. These BIBD are shown in Table 1.3 and Table 1.4 respectively.

$\begin{array}{llllllllllll}B_{1} & B_{2} & B_{3} & B_{4} & B_{5} & B_{6} & B_{7} & B_{8} & B_{9} & B_{10} & B_{11} & B_{12} \\ 1 & 4 & 7 & 1 & 2 & 3 & 1 & 2 & 3 & 1 & 2 & 3 \\ 2 & 5 & 8 & 4 & 5 & 6 & 5 & 6 & 4 & 6 & 4 & 5 \\ 3 & 6 & 9 & 7 & 8 & 9 & 9 & 7 & 8 & 8 & 9 & 7\end{array}$

Table 1.3: $(9,3,1)-B I B D[13]$

\begin{tabular}{lllllllllllll}
$\mathrm{B}_{1}$ & $\mathrm{~B}_{2}$ & $\mathrm{~B}_{3}$ & $\mathrm{~B}_{4}$ & $\mathrm{~B}_{5}$ & $\mathrm{~B}_{6}$ & $\mathrm{~B}_{7}$ & $\mathrm{~B}_{8}$ & $\mathrm{~B}_{9}$ & $\mathrm{~B}_{10}$ & $\mathrm{~B}_{11}$ & $\mathrm{~B}_{12}$ & $\mathrm{~B}_{13}$ \\
1 & 2 & 3 & 4 & 5 & 6 & 7 & 8 & 9 & 10 & 11 & 12 & 13 \\
3 & 4 & 5 & 6 & 7 & 8 & 9 & 10 & 11 & 12 & 13 & 1 & 2 \\
9 & 10 & 11 & 12 & 13 & 1 & 2 & 3 & 4 & 5 & 6 & 7 & 8 \\
\hline $\mathrm{B}_{14}$ & $\mathrm{~B}_{15}$ & $\mathrm{~B}_{16}$ & $\mathrm{~B}_{17}$ & $\mathrm{~B}_{18}$ & $\mathrm{~B}_{19}$ & $\mathrm{~B}_{20}$ & $\mathrm{~B}_{21}$ & $\mathrm{~B}_{22}$ & $\mathrm{~B}_{23}$ & $\mathrm{~B}_{24}$ & $\mathrm{~B}_{25}$ & $\mathrm{~B}_{26}$ \\
2 & 3 & 4 & 5 & 6 & 7 & 8 & 9 & 10 & 11 & 12 & 13 & 1 \\
6 & 7 & 8 & 9 & 10 & 11 & 12 & 13 & 1 & 2 & 3 & 4 & 5 \\
5 & 6 & 7 & 8 & 9 & 10 & 11 & 12 & 13 & 1 & 2 & 3 & 4
\end{tabular}

Table 1.4: (13,3,1)-BIBD 
Theorem 1.3. If $\left\{D_{1}, \ldots, D_{t}\right\}$ is a $(v, k, \lambda)$-difference family in a group $G$ then there exists a $(v, k, \lambda)$-BIBD.

Proof. Let $\left\{D_{1}, \ldots, D_{t}\right\}$, be a $(v, k, \lambda)$-difference family in a group $G$. Let $B_{i j}=D_{1}+j=\left\{d+j: d \in D_{i}\right\}$. We show that $\left(G,\left\{B_{i j}: 1 \leq i \leq t, j \in G\right\}\right)$ is a $(v, k, \lambda)$-BIBD. Suppose $x, y \in G$ and let $d=x-y$. By definition of the $(v, k, \lambda)$, every non-identity elements is covered by a difference of elements in some $D_{i}$ exactly $\lambda$ times. For $1 \leq l \leq \lambda$ let $d_{l_{1}}, d_{l_{2}} \in D_{i_{l}}$ with $d=d_{l_{1}}-d_{l_{2}}$. Then we have $x, y \in B_{i_{l},\left(x-d_{l_{1}}\right)}$.

Let $(X, \mathcal{B})$ be a $(v, k, \lambda)$-BIBD and $0 \leq i \leq k$. The $i$-block intersection graph of $(X, \mathcal{B})$ is a graph $G$ such that $\mathrm{V}(G)=\mathcal{B}$ and an edge $e \in E$ joins two vertices (blocks) if they have exactly $i$ elements $x \in X$ in common. The block intersection graph is the union of $i$-blocks for $i>0[16]$.

The 1-intersection graph of the Fano Plane is given in Table 1.1. Each pair of block shares exactly one element in common so the 1-intersection graph of the Fano Plane is $K_{7}$.

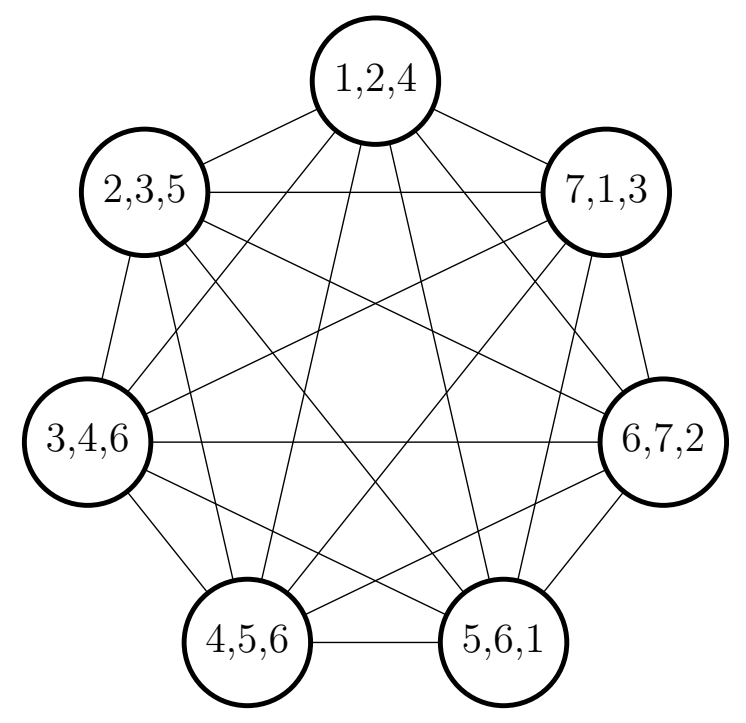

Figure 1.3: The block intersection graph of STS(7)

\subsection{Literature Review}

Single change covering designs differ from BIBDs and Steiner Systems. A single change covering design $(\operatorname{SCCD}(v, k, s, b))(X, \mathcal{L})$ is a $v$-set $X$ and 
an ordered list of blocks $\mathcal{L}=\left(B_{1}, B_{2}, \ldots, B_{b}\right)$ of size $k$ where every $s$-set, $S$, must occur in at least one block. Every block except the last differs from the next block by removing one element and introducing another. That is $\left|B_{i} \cap B_{i+1}\right|=(k-1)$ for all $1 \leq i<b$ and the point $x=B_{i} \backslash B_{i+1}$ is removed and $y=B_{i+1} \backslash B_{i}$ is introduced in $B_{i+1}$. If $\left|B_{1} \cap B_{b}\right|=k-1$ then the SCCD is circular, denoted CSCCD. If the SCCD is circular one point is introduced in each block. If the SCCD is non-circular every point in $B_{1}$ is introduced and one point is introduced in each subsequent block. A $s$-set, $S$, is covered on block $B_{i}$ if $S \subseteq B_{i}$ and one element of $S$ was introduced in $B_{i}$.

An example $\operatorname{SCCD}(7,3,2,10)$ is found in Table 1.5. If no $\operatorname{SCCD}\left(v, k, s, b^{\prime}\right)$ exists for all $b^{\prime}<b$ then a $\operatorname{SCCD}(v, k, s, b)$ is called minimal. We emphasize the introduced element in each block with an asterisk, *. Note that 1,2 , and 3 are all introduced in the first block so the pairs $\{1,2\},\{1,3\},\{2,3\}$ are covered in $B_{1}$. In $B_{2}$ only 4 is introduced so only the pairs involving 4 are covered, namely $\{1,4\},\{2,4\}$. Table 1.6 shows an example of a $\operatorname{CSCCD}(6,3,2,8)$ where the pair $\{1,4\}$ is covered two times.

$\begin{array}{llllllllll}B_{1} & B_{2} & B_{3} & B_{4} & B_{5} & B_{6} & B_{7} & B_{8} & B_{9} & B_{10} \\ 1^{*} & 1 & 1 & 6^{*} & 7^{*} & 7 & 4^{*} & 4 & 4 & 1^{*} \\ 2^{*} & 2 & 2 & 2 & 2 & 3^{*} & 3 & 3 & 7^{*} & 7 \\ 3^{*} & 4^{*} & 5^{*} & 5 & 5 & 5 & 5 & 6^{*} & 6 & 6\end{array}$

Table 1.5: $\operatorname{SCCD}(7,3,2,10)$

$\begin{array}{llllllll}B_{1} & B_{2} & B_{3} & B_{4} & B_{5} & B_{6} & B_{7} & B_{8} \\ 6^{*} & 6 & 6 & 6 & 4^{*} & 4 & 2^{*} & 2 \\ 4 & 3^{*} & 3 & 3 & 3 & 5^{*} & 5 & 4^{*} \\ 2 & 2 & 5^{*} & 1^{*} & 1 & 1 \wedge & 1 & 1 \wedge\end{array}$

Table 1.6: $\operatorname{CSCCD}(6,3,2,8)$. The pair $\{1,4\}$ is covered in $B_{5}$ and $B_{8}$. [8]

We say that the set of elements that remain the same between $B_{i}$ and $B_{i+1}$ of a SCCD is the $i^{\text {th }}$ unchanged subset between these blocks, $U_{i}=$ $B_{i} \cap B_{i+1}[12]$. If the SCCD is not circular, we also say that any $(k-1)$ subset of $B_{1}$ or $B_{b}$ is an unchanged subset $U_{0}$ or $U_{b}$ respectively. Consider the $\operatorname{SCCD}(7,3,2,10)$ in Table 1.5; the unchanged subsets are $U_{1}=\{1,2\}$, $U_{2}=\{1,2\}, U_{3}=\{2,5\}, U_{4}=\{2,5\}, U_{5}=\{7,5\}, U_{6}=\{3,5\}, U_{7}=\{4,3\}, U_{8}=\{4$, 
6\}, $U_{9}=\{7,6\}$. Additionally $U_{0}$ could be $\{1,2\},\{1,3\}$, or $\{2,3\}$, and $U_{10}$ could be $\{1,7\},\{6,7\}$, or $\{1,6\}$.

If there exists $\left\{i_{j}, 1 \leq j \leq \frac{v}{k-1}\right\}$ such that

$$
X=\bigcup_{j=1}^{\frac{v}{k-1}} U_{i_{j}}
$$

then we say that $\mathcal{E}=\left\{U_{i_{1}}, U_{i_{2}}, \ldots, U_{i_{\left(\frac{v}{(k-1)}\right.}}\right\}$ is an expansion set. If the SCCD is not circular and $\mathcal{E}$ contains $u_{0}, u_{b}$ or both, then $\mathcal{E}$ is an outer expansion set. If $\mathcal{E}$ contains neither $u_{0}$ or $u_{b}$ then we say $\mathcal{E}$ is an inner expansion set. We will denote expansion set locations in our tables with carets, $\wedge$. Consider Table 1.6, the carets between blocks 3 and 4, blocks 6 and 7 , and after block 8 denote the expansion set where $U_{i_{1}}=U_{3}=\{3,6\}$, $U_{i_{2}}=U_{6}=\{1,5\}$, and $U_{i_{3}}=U_{8}=\{2,4\}$. Now $X=U_{i_{1}} \dot{\cup} U_{i_{2}} \dot{\cup} U_{i_{3}}$ so this is a valid expansion set.

The earliest discussion of SCCDs was in 1968 and focused on applications to efficient computing [9]. Suppose we want to calculate $A A^{T}$ for some matrix. We notice that the rows of $A$ and columns of $A^{T}$ are the same; so we must process each pair of rows in $A$. In the 1960's RAM (Random Access Memory) consisted of magnetic-core memory or magnetic-rope memory and held $4 \mathrm{~KB}$. This $4 \mathrm{~KB}$ worth of memory cost $\$ 320$ in 1969 or about $\$ 2,240$ in 2021 , and could be read at $1 \mathrm{MB} / \mathrm{s}$. This meant we would not be able to store all the data we need to process in RAM through the whole calculation when the matrix is large. Thus, we supplement this fast memory with tape-drive memory. Tape-drive memory is much less expensive and held $46 \mathrm{MB}$ per reel but was read at $15 \mathrm{~KB} / \mathrm{s}$. A naive program would move the relevant rows into the magnetic-core from the tape-drive and process the matrix by removing and adding rows in numerical order. However, reading information from the tape-drive to RAM would take a long time. Nelder sought to minimize reading from the tape-drive by methodically removing and adding the rows to RAM by using SCCD.

Gower and Preece continued the discussion in 1972 when they looked at successive incomplete blocks. The area was left alone after this until the 1990s when the group of Preece, Constable, Zhang, Yucas, Wallis, McSorley and Phillips re-started the discussion and a series of eleven papers substantially developed the theory. In 1992 [15] Yucas, Wallis and Zhang derived bounds on the number of blocks in a $\operatorname{SCCD}(v, k, 2, b)$ and gave a construction for optimal SCCD with $k=3$. In 1993 Rees [14] discussed upper bounds 
on $\operatorname{SCCD}(v, k, 2, b)$ for $k \geq 4$. In 1994 Zhang [18] introduced the idea of a missing pair (hole) from general design theory to single change covering designs. He also refined some new bounds on the number of blocks a SCCD can have. In 1995 [12] Preece, Constable, Zhang, Yucas, Wallis, McSorley, and Phillips essentially solved the existence of minimal SCCD with $k=4$ using expansion sets. They also mentioned an extension to a double change covering design and gave a single example of a tight $\operatorname{DCCD}(9,3,2,12)$. In 1999 Phillips and Preece [11] found all $2554 \operatorname{SCCD}(12,4,2,21)$ and show that there are 566 which are not minor variants of each other. Philips [10] closed this period in 2000 by finding two tight $\operatorname{SCCD}(20,5,2,46)$ using optimized code, a major breakthrough as the size of the search space grows exponentially as $k$ increases.

During this period in 1998 McSorley also explored circular single change covering designs [8]. He completely solved minimal $\operatorname{CSCCD}(v, k, 2, b)$ where $k=2,3$. He gave some lower bounds on the number of blocks in a circular design and some families of circular designs with a fixed $k$. Analysing properties of elements in a design, he explored substructures that must be present in circular designs to significantly reduce the work required in a computer search which lead to him constructing all the minimal $\operatorname{CSCCD}(9,4,2,12)$ and $\operatorname{CSCCD}(10,4,2,15)$. He ended this paper by discussing the non-existence of some tight SCCD.

I re-opened research on SCCD in my undergraduate honours thesis by formalizing a theorem given in [12] and expanding its utility to CSCCD and SCCD with some pairs covered more then once. I proved that all minimal circular $\operatorname{SCCD}(v, 4,2, b)$ and around half the possible circular and non-circular $\operatorname{SCCD}(v, 5,2, b)$ exist.

Let $g_{1}(v, k, 2)=\frac{\left(\begin{array}{c}v \\ 2\end{array}\right)-\left(\begin{array}{c}k \\ 2\end{array}\right)}{k-1}+1$ and $g_{2}(v, k, 2)=\frac{\left(\begin{array}{c}v \\ 2\end{array}\right)}{k-1}$. Wallis et al showed.

Lemma 1.4. [15] In a $S C C D(v, k, 2, b), b \geq g_{1}(v, k, 2)$. In a circular $S C C D(v, k, 2, b), b \geq g_{2}(v, k, 2)$.

We say a (circular) $\operatorname{SCCD}(v, k, 2, b)$ is economical if it has $\left\lceil g_{1}(v, k, 2)\right\rceil$ $\left(\left\lceil g_{2}(v, k, 2)\right\rceil\right)$ blocks. In other words, it has the fewest number of blocks possible for this $v$ and $k$. A (circular) tight if it is economical and $g_{1}(v, k, 2)$ $\left(g_{2}(v, k, 2)\right)$ is an integer. In a tight SCCD every pairs is covered exactly once. With some $v, k$ combinations it will be impossible to create tight SCCD, in these cases the economical design is our best option. A SCCD of strength two is economical if and only if no more than $k-1$ pairs are repeatedly covered, counting multiplicities. 
It is worth noting that $\operatorname{SCCD}(v, k, 2, b)$ do require more blocks to build than $\mathrm{S}(2, k, v)$. However, when used to test components, SCCD offer savings if exchanging components between tests is more expensive than conducting each test. Recall the earlier scenario where a company is testing 7 variations of a component. Suppose the company is under a deadline and swapping components between tests costs the company 20 minutes, but running a test only requires 1 minute. A company using a $(7,3,1,7)$-BIBD would need to swap components out 15 times and run 7 tests, costing them 307 minutes. A company using a $\operatorname{SCCD}(7,3,2,7)$ would need to change components 12 times and run 10 tests, costing them 250 minutes. Helping to make statistical computing more efficient motivated Nelder, Gower and Preece in their early investigations. SCCD can help make statistical computing computations more efficient [6].

Wallis, Yucas and Zhang proved

Lemma 1.5. [15] There is an economical $\operatorname{SCCD}(v, k, 2, b)$ whenever $k=$ $v, v-1$, or $v$ is odd and $k=2$.

When $v=k, b=1$. When $k=v-1, b=3$. When $k=2$ and $v$ is odd, $b=\left(\begin{array}{l}v \\ 2\end{array}\right)$. For $k=3$, they give a recursive construction.

Lemma 1.6. [15] If there is a $\operatorname{SCCD}(v, 3,2, b)$ then there is a $S C C D(v+$ $\left.4,3,2, b^{\prime}\right), b^{\prime}=2 v+3+b$.

Using Lemma 1.6 they built an economical SCCD for any $v \geq 3$.

Theorem 1.7. [15] There is an economical $\operatorname{SCCD}(v, 3,2, b)$ for all $v \geq 3$.

They prove another lower bound on $b$ by utilising the number of required introductions.

Theorem 1.8. [15] If $S C C D(v, k, 2, b)$ exists then $b \geq g_{3}(v, k, 2)=2(v-$ $k)+1$. Furthermore, if $k \geq 4$ and $v=3 k-3$ then $b \geq 4 k-4$ blocks.

They proved a number of recursive constructions.

Theorem 1.9. [15] Suppose there exists a $\operatorname{SCCD}(v, k, 2, b)$. There exists a $S C C D\left(v^{\prime}, k, 2, b^{\prime}\right), v^{\prime}=v+2 k-2, b^{\prime}=b+2 v+3 k-6$.

Lemma 1.10. [15] The number of blocks in a $S C C D(v, k, 2, b)$ is at least $b=\left\lceil\frac{\left(\begin{array}{l}v \\ 2\end{array}\right)-\left(\begin{array}{c}k-1 \\ 2\end{array}\right)}{k-1}\right\rceil$ 
Theorem 1.11. [15] Suppose a $S C C D(v-j, k, 2, b)$ exists and $j \leq k-1$. Then a $S C C D(v, k, 2, b+v-k+j)$ exists.

They consider restrictions on block size by small $v$, a corollary of Theorem 1.8 .

Theorem 1.12. [15] For a $\operatorname{SCCD}(v, k, 2, b)$, if $2 k \leq v \leq 3 k-3$ then $b \leq$ $3 v-4 k+1$.

Corollary 1.13. [15] Given a $S C C D(v, k, 2, b)$, if $v \leq 2 k$ then $b=2(v-k)+1$

In 1993 Rees uses the frequency and introduction limits to show that :

Theorem 1.14. [14] If $k \geq 4$ and $a \operatorname{SCCD}(3 k-2, k, 2, b)$ exists then $b \geq$ $4 k-1$.

In 1994 Zhang shows the following in parallel to a theorem of [15].

Lemma 1.15. [18] In a $S C C D(v, k, 2, b), b \geq\left\lceil\frac{v(v-1)-(k-1)(k-2)-2}{2(k-1)}\right\rceil$ with equality if $k=2,3$.

He then shows an upper bound when $v=n(k-1)+i$ and $k+1 \leq i \leq 2 k-1$, $n \geq 0$, and $k \geq 4$ for $d(v, k)=2(i-k)+n(i+k-3)+\frac{n(n-1)(k-1)}{2}$.

A single change covering design with a hole, $\operatorname{SCCDH}(v, k, 2, v)$ is a $\operatorname{SCCD}(v, k, 2, b)$ such that every pair of elements of $V$ occur on at least one block except for one pair, $\{x, y\}$, called a hole such that one of $x$ and $y$ appear in $B_{1}$ or $B_{b}$. We use $f^{\prime}(v, k, 2)$ to denote the smallest $b$ for which there exists a $\operatorname{SCCDH}(v, k, 2, b)$.

Theorem 1.16. [18] It holds that $f^{\prime}(v, k, 2) \leq d(v, k)$.

Corollary 1.17. [18]

1. $f^{\prime}(v, 4,2)=\left\lceil\frac{v^{2}-v-8}{6}\right\rceil$ for all $v \geq 5$.

2. $\left\lceil\frac{v^{2}-v-6}{6}\right\rceil \leq g_{1}(v, 4,2) \leq\left\lceil\frac{v^{2}-v-2}{6}\right\rceil$ for all $v \geq 5$. In particular, $g_{1}(v, 4,2)=\frac{v^{2}-v-2}{6}$ if $v \equiv 2(\bmod 3)$

Theorem 1.18. [18] $g_{1}(v, k, 2) \geq 3 v-4 k+1$ with equality if $k \geq 4$ and $2 k+1 \leq v \leq 3 k-1$. 
Corollary 1.19. [18] $g_{1}(v, k, 2) \geq 2(v-k)+1$ with equality if and only if $v \leq 2 k$ and $(v, k) \neq(4,2)$.

Theorem 1.20. [18] $g_{1}(m v, m k, 2) \leq\lceil f(v, k)-1\rceil m+1$ for any positive integer $m$.

Corollary 1.21. [18] If $g_{1}(v, k, 2)=3 v-4 k+1$ then $g_{1}(m v, m k, 2)=$ $3 m v-4 m k+1$ for every positive integer $m$.

Theorem 1.22. [18] It holds that $g_{1}(v+2, k+1,2) \leq g_{1}(v, k, 2)+2$.

Corollary 1.23. [18]

1. $g_{1}(v+2 m, k+m, 2) \leq g_{1}(v, k+2 m, 2)$ for all $m \geq 1$

2. If $g_{1}(v, k, 2)=3 v-4 k+1$ then $g_{1}(v+2 m, k+m, 2)=3(v+2 m)-$ $4(k+m)+1$ for every positive integer $m$.

In 1995 Preece et al. further considered tight single change covering designs [12]. They found a recursive construction for tight single change covering designs utilizing expansion sets. The proof is easily extended to economical SCCD.

Proposition 1.24. If a (economical) $\operatorname{SCCD}(v, k, 2, b)$ with an expansion set exists, then a (economical) $S C C D\left(v+1, k, 2, b+\frac{v}{k-1}\right)$ exists.

Theorem 1.25. For $k \geq 4$ if a tight $\operatorname{sccd}(v, k)$ with $b$ blocks and an outer expansion set exists then a economical $\operatorname{sccd}(v+2, k)$ with $\left(b+2 \frac{v}{k-1}+1\right)$ blocks exists if $v+2$ is an admissible value for an economical design $(v+2, k)$.

They state this recursive construction informally. I formalized this and gave an explicit proof in my undergraduate thesis [3].

Theorem 1.26. If there exists a tight $S C C D(v, k, 2, b)$ and $a$ tight $S C C D\left(n(k-1), k, 2, b^{\prime}\right)$ with an outer expansion set, then there exists a tight $S C C D\left(v+(n-1)(k-1), k, 2, b^{*}\right)$, with $b^{*}=b+b^{\prime}+\frac{v(v-k+1)}{k-1}$. Furthermore, if the $S C C D(v, k, 2, b)$ has an expansion set then the $S C C D(v+(n-1)(k-$ $\left.1), k, 2, b^{*}\right)$ has an expansion set.

I generalized Theorem 1.26 to construct economical SCCD. We say that a block $B_{i}$ in a SCCD is tight if the pairs it covers are not covered in any other block of the SCCD. tight blocks allow us to use one economic SCCD and one tight SCCD to construct larger economic designs using the following lemma. 
Lemma 1.27. [3] Let $\left(X, \mathcal{L}=\left(B_{1}, \ldots, B_{b}\right)\right)$ be a $\operatorname{SCCD}(v, k, 2, b)$ and let $d=(k-1) b+\left(\begin{array}{c}k-1 \\ 2\end{array}\right)-\left(\begin{array}{c}v \\ 2\end{array}\right)$. Suppose that $\left(X^{\prime}, \mathcal{L}^{\prime}=\left(B_{1}, \ldots, B_{b}, B_{b+1}^{\prime}, \ldots, B_{b^{\prime}}^{\prime}\right)\right)$ is a $S C C D\left(v^{\prime}, k, 2, b^{\prime}\right)$ and $X \subseteq X^{\prime}$. If $B_{i}^{\prime}$ is tight $\forall i, b+1 \leq i \leq b^{\prime}$, then $\left(X^{\prime}, \mathcal{L}^{\prime}\right)$ is economical and has $d=(k-1) b^{\prime}+\left(\begin{array}{c}k-1 \\ 2\end{array}\right)-\left(\begin{array}{c}v^{\prime} \\ 2\end{array}\right)$.

In Lemma $1.27 d$ is a measure of tightness. Some $\operatorname{SCCD}(v, k, 2, b)$ require that up to $k-2$ pairs be covered more then once in order cover all pairs. Consider Table 1.7 and Table 1.8, they make up the economic $\operatorname{SCCD}(8,3,2,14)$ in Table 1.9

$$
\begin{array}{rll}
1^{*} & 4^{*} & 4 \\
2^{*} & 2 & 1^{*} \\
3^{*} & 3 & 3 \wedge
\end{array}
$$

Table 1.7: economical $\operatorname{SCCD}(4,3,2,3)$

$\begin{array}{lllllllllll}4^{*} & 7^{*} & 7 & 7 & 7 & 7 & 7 & 6^{*} & 6 & 6 & 6 \\ 8^{*} & 8 & 8 & 8 & 8 & 8 & 4^{*} & 4 & 1^{*} & 2^{*} & 3^{*} \\ 3^{*} & 3 & 6^{*} & 1^{*} & 2^{*} & 5^{*} & 5 & 5 & 5 & 5 & 5\end{array}$

Table 1.8: Tight single change blocks

$\begin{array}{rlllllllllllll}1^{*} & 4^{*} & 4 & 4 & 7^{*} & 7 & 7 & 7 & 7 & 7 & 6^{*} & 6 & 6 & 6 \\ 2^{*} & 2 & 1^{*} & 8^{*} & 8 & 8 & 8 & 8 & 8 & 4^{*} & 4 & 1^{*} & 2^{*} & 3^{*} \\ 3^{*} & 3 & 3_{\wedge} & 3 & 3 & 6_{\wedge}^{*} & 1^{*} & 2^{*} & 5^{*} & 5 & 5 & 5 & 5 & 5_{\wedge}\end{array}$

Table 1.9: economical $\operatorname{SCCD}(8,3,2,14)$

Theorem 1.28. [3] If there exists an economical $\operatorname{SCCD}(v, k, 2, b)$ and $a$ tight $S C C D\left(n(k-1), k, 2, b^{\prime}\right)$ with an outer expansion set, then an economical $S C C D\left(v+(n-1)(k-1), k, 2, b^{*}\right), b^{*}=b+b^{\prime}+\frac{v(v-k+1)}{k-1}$ exists. Furthermore, if the economical $\operatorname{SCCD}(v, k, 2, b)$ has an expansion set then the economical $S C C D\left(v+(n-1)(k-1), k, 2, b^{*}\right)$ will have an expansion set.

In 2000 Phillips used exhaustive search with the following three optimization Lemmas to find a $\operatorname{SCCD}(20,5,2,46)$. Consider the tight $\operatorname{SCCD}(12,4,2,21)$ from [10] in Table 1.10 for the following. First Phillips defines $B_{i}, B_{i+1} \in \mathcal{L}$ to have the minor variant property if $x$ is introduced in $B_{i}$ and removed 
in $B_{i+1}$ and $y$ is introduced in $B_{i+1}$ and removed in $B_{i+2}$. For $i=b-1$, we consider block $B_{b+1}=\{\}$, so all $x \in B_{b}$ are removed in $B_{b+1}$. For example in Table 1.10, 1 is introduced in $B_{13}$ and removed in $B_{14}$ and 3 is introduced in $B_{14}$ and removed in $B_{15}$. There is a second example in $B_{20}$ and $B_{21}$ with elements 4 and 9 .

Lemma 1.29. [10] If a $S C C D(v, k, 2, b),(X, \mathcal{L})$, has a minor variant in $B_{i}$ then swapping $B_{i}$ and $B_{i+1}$ produces a $S C C D(v, k, 2, b)$.

Phillips takes advantage of Lemma 1.29 in an exhaustive search by noticing we only need find one of each pair of minor variant SCCD. His search only advances on a particular SCCD with a minor variant $x \in B_{i}, y \in B_{i+1}$ if $x<y$. The earlier a minor variant occurs in the design the more significant the computational savings.

In a $\operatorname{SCCD}(v, k, 2, b),(X, \mathcal{L})$, let $Z \subseteq X$ and $x, y \in X \backslash Z$. If the pairs $\{z, x\},\{z, y\} \forall z \in Z$ are covered in $\left.B_{1}, \ldots, B_{i}\right)$ we say that $B_{i}$ has the end permutation property. Block $B_{8}$ with $x=4, y=5$ in Table 1.10 , has the end permutation property. We can swap the points 4 and 5 in all blocks $B_{8}, \ldots, B_{21}$ and produce another SCCD.

Lemma 1.30. [10] If $B_{i}$ in a $S C C D(v, k, 2, b),(X, \mathcal{L})$, has the end permutation property with elements $x, y$ then we may swap $(x, y)$ in $B_{i}, \ldots, B_{b}$ to produce a $\operatorname{SCCD}(v, k, 2, b)$.

Once again Phillips optimizes his search by checking for end permutations and only continuing the search if $x<y$ is introduced first in $B_{i}, \ldots, B_{b}$.

Finally, he considers the implications of removing an element from a block. When an element is removed, if it still needs to be reintroduced to cover a pair, the next introduction will cover $k-1$ pairs. If there are at least one and fewer than $k-1$ pairs left to cover with point $x$ we know that $x$ may not be removed as doing so will never produce a tight SCCD. For example, in $B_{16}$ of Table 1.10 we cannot remove 7 as we have yet to cover $\{7,5\}$, however this is the only pair involving 7 yet to be covered, so 7 must be in $B_{16}$. We call this checking the removal conditions of an element.

Lemma 1.31. [10] In a tight $S C C D(v, k, 2, b),(X, \mathcal{L})$ if $x \in B_{i}$ and at least $v-k+1$ and less than $v-1$ pairs involving $x$ are covered in $B_{1}, \ldots, B_{i}, x$ is not removed from $B_{i}$. 


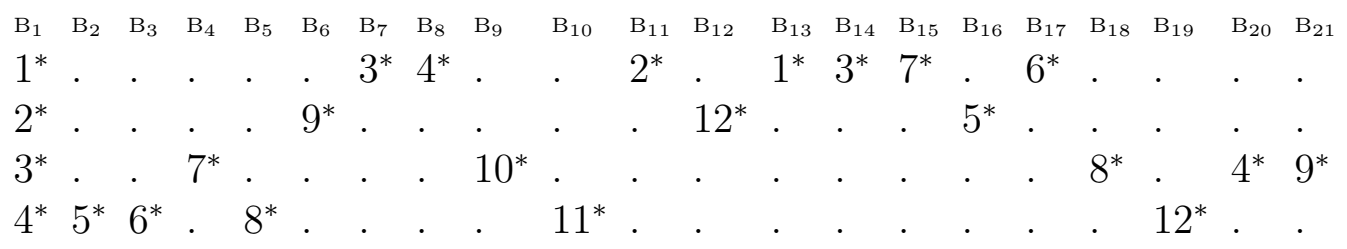

Table 1.10: $\operatorname{SCCD}(12,4,2,21)[10]$

With these improvements to his search and using multiple CPUs, Phillips found two $\operatorname{SCCD}(20,5,2,26)$.

Using these $\operatorname{SCCD}(20,5,2,46)$, I was able to build a significant portion of tight circular and non-circular $\operatorname{SCCD}(v, 5,2, b)$ as seen in Theorem 1.40. We will discuss this methodology further in Section 2.1 when we discuss how we incorporated this in our recursive search.

In 1999 McSorley investigated circular SCCD. He proved the existence for all economic $\operatorname{CSCCD}(v, k, 2, b)$ for $k=2,3$.

Theorem 1.32. An economic $\operatorname{CSCCD}(v, k, 2, b)$ exists for all $v$ and $k=2,3$. These designs are tight if $k=2$ or if $k=3$ and $v \equiv 0$ or $1(\bmod 4)$.

$\mathrm{He}$ also finds some restrictions on the minimum number of blocks and constructions of families of CSCCD for small $v$. Let $B_{0}=\{0, \ldots, k-1\} \in \mathbb{Z}_{n}$, $B_{i}=B_{0}+i$ and $B_{i}^{\prime}=B_{i} \cup\{\infty\}$.

Theorem 1.33. [8]

1. For $k \geq 3$ and $k \leq v \leq 2 k-2$ the blocks $\mathcal{B}=\left(B_{1}^{\prime}, B_{2}^{\prime}, \ldots, B_{b}^{\prime}\right)$ form an economical circular $C S C C D(v, k, 2, b)$ over $\mathbb{Z}_{v-1} \cup \infty$. When $v=2 k-2$ this CSCCD is tight.

2. For $k \geq 2\left(\mathbb{Z}_{2 k-1},\left(B_{i}\right)\right)$ is a tight circular $\operatorname{SCCD}(2 k-1, k, 2,2 k-1)$.

3. For $k \geq 3$ and $v=2 k$ let $C=B_{k-1} \backslash b_{k} \cup\{\infty\}, C^{\prime}=B_{2 k-2} \backslash 0 \cup\{\infty\}$, and $C^{\prime \prime}=C^{\prime} \backslash(2 k-1) \cup\{0\}$ the blocks $\mathcal{B}=\left(B_{0}, \ldots, B_{k-1}, C, B_{k}, \ldots, B_{2 k-2}, C^{\prime}, C^{\prime \prime}\right)$ form an economical circular $S C C D(2 k, k, 2,2 k+2)$. If $k=2$ then this design is tight.

Using the concept of expansion sets with circular $\operatorname{SCCD}(v, k, 2, b)$ he was able to construct circular SCCD with exactly one extra element.

McSorley used the obvious extension of Proposition 1.24 to circular SCCD, specifically when moving from $\operatorname{CSCCD}(9,4,2,11)$ to $\operatorname{CSCCD}(10,4,2,14)$. This holds true, for all economical circular SCCD. 
Proposition 1.34. If an economical $C S C C D(v, k, 2, b)$ with an expansion set exists then a circular economical CSCCD $\left(v+1, k, 2, b+\frac{v}{k-1}\right)$ exists. If the $\operatorname{CSCCD}(v, k, 2, b)$ is tight then so is the $\operatorname{CSCCD}\left(v+1, k, 2, b+\frac{v}{k-1}\right)$.

McSorley considered circular single change covering designs and developed SF-arrays and skeletons which significantly reduced the work in algorithmic searches for circular $\operatorname{SCCD}[8]$. Let $(X, \mathcal{L})$ be a tight $\operatorname{CSCCD}(v, k, 2$, $b$ ). We denote the set of elements introduced $i$ times in $\mathcal{L}$ by $T_{i}$ and $t_{i}$ is the cardinality of $T_{i}$. We denote the number of blocks containing the element $x$ by $f_{x}$.

Note that for $x \in T_{0}, x$ is never introduced but pairs containing $x$ must be covered, so $x$ must be in every block. If $x, y \in T_{0}$ then $\{x, y\}$ is never covered therefore $t_{0}=0$ or 1 . McSorley showed that tight $\operatorname{CSCCD}(v, k, 2, b)$ with $t_{0}=1$ must have $t_{1}=v-1$ and consequently $v=2 k-2, b=2 k-3$.

In a tight CSCCD we know that each time $x \in T_{i}$ is introduced we will cover $k-1$ pairs that contain $x$. As there are $v-1$ pairs in $X$ that contain $x$ we have for non-empty $T_{i}$ that $i \leq M=\left\lfloor\frac{v-1}{k-1}\right\rfloor$. We say $M$ is the maximum introduction limit. McSorley proved the following.

Theorem 1.35. In a tight $C S C C D(v, k, 2, b)[8]$

1. $t_{i}=0 \forall i>M$

2. $\sum_{i=1}^{A} t_{i}=v$

3. $\sum_{i=1}^{A} i t_{i}=b$

4. $f_{\{x\}}=f_{i}=(v-1)-i(k-2) \forall x \in T_{i}$

5. If $f_{i}=i$ then $t_{i}=0$ or 1

6. If $f_{i}=i+1$ then $t_{i}=0,1$, or 2 .

Note that when we use a set notation in the subscript of $f_{\{x\}}$, we are talking about the frequency of element $x$. However if we use a number in the subscript of $f_{i}$, we are talking about the frequency of all elements introduced $i$ times.

McSorley analyzed the introductions and removals of elements in $T_{1}$. For example consider the tight $\operatorname{CSCCD}(8,3,2,14)$ in Table 1.11 . We see that $T_{1}=\{0,1\}, T_{2}=\{2,3,4,5,6,7\}$ and observe 0 and 1 are both in $T_{1}$ and the 
pair $\{0,1\}$ must be covered. Therefor 1 is introduced before 0 removed or visa versa.

$\begin{array}{llllllllllllll}\mathrm{B}_{1} & \mathrm{~B}_{2} & \mathrm{~B}_{3} & \mathrm{~B}_{4} & \mathrm{~B}_{5} & \mathrm{~B}_{6} & \mathrm{~B}_{7} & \mathrm{~B}_{8} & \mathrm{~B}_{9} & \mathrm{~B}_{10} & \mathrm{~B}_{11} & \mathrm{~B}_{12} & \mathrm{~B}_{13} & \mathrm{~B}_{14} \\ 0 & 0 & 0 & 5^{*} & 6^{*} & 6 & 6 & 4^{*} & 4 & 5^{*} & 5 & 5 & 5 & 2^{*} \\ 1^{*} & 1 & 1 & 1 & 1 & 1 & 7^{*} & 7 & 7 & 7 & 6^{*} & 6 & 3^{*} & 3 \\ 2 & 7^{*} & 4^{*} & 4 & 4 & 3^{*} & 3 & 3 & 2^{*} & 2 & 2 & 0^{*} & 0 & 0\end{array}$

Table 1.11: tight $\operatorname{CSCCD}(8,3,2,14)$

For any given $x \in T_{1}$ we say that $x$ starts, $S$, (is introduced), in block $B_{x, 1}$ and finishes, $F$, in block $B_{x, f_{1}}$. We call the successive blocks $B_{x, i}$ containing $x$ the run of $x$. We also know that in general for every pair to be covered, each other element in $T_{1}$ must either finish or start but not both during the run of $x$. For example, in Table 1.11, 0 is introduced in $\mathrm{B}_{12}$ and is removed in $\mathrm{B}_{4}$ after 1 is introduced in $\mathrm{B}_{1}$.

The SF-array of $x$ is a list, $A$, of length $f_{1}$ with entries "-", "S", "F", or "SF" which describes how all elements in $T_{1}$ must interact in relation to a single element $x \in T_{1}$. If $y \in T_{1}$ is introduced in block $B_{x, i+j}, A_{i+j}$ contains a " $\mathrm{S}$ ". If $y \in T_{1}$ is present in $\mathrm{B}_{x, i+j}$ but not $\mathrm{B}_{x, i+j+1}, A_{i+j}$ contains an "F". For the tight $\operatorname{CSCCD}(8,3,2,14) f_{1}=6$ and in Table 1.11 a SF-arrays based on the element 0 and 1 are

\begin{tabular}{ccc|ccc} 
& SF-array & wt(i) & & SF-array & wt(i) \\
\hline $\mathrm{R}_{0,1}$ & $\mathrm{~S}$ & 1 & $\mathrm{R}_{1,1}$ & $\mathrm{~S}$ & 2 \\
$\mathrm{R}_{0,2}$ & - & 1 & $\mathrm{R}_{1,2}$ & - & 2 \\
$\mathrm{R}_{0,3}$ & - & 1 & $\mathrm{R}_{1,3}$ & $\mathrm{~F}$ & 2 \\
$\mathrm{R}_{0,4}$ & $\mathrm{~S}$ & 2 & $\mathrm{R}_{1,4}$ & - & 1 \\
$\mathrm{R}_{0,5}$ & - & 2 & $\mathrm{R}_{1,5}$ & - & 1 \\
$\mathrm{R}_{0,6}$ & $\mathrm{~F}$ & 2 & $\mathrm{R}_{1,6}$ & $\mathrm{~F}$ & 1 \\
& $x=0$ & & & $x=1$ &
\end{tabular}

Table 1.12: SF-arrays for tight $\operatorname{CSCCD}(8,3,2,14)$

We will write $S S$ to indicate the event that $\mathrm{B}_{i}$ and $\mathrm{B}_{i+1}, i<f_{1}$, both contain an $S$ and similarly for $F F$. Let $n_{S}$ be the number of "S" and $n_{F}$ be the number of "F" in $A$. Let $n_{S S}$ be the number of "SS" events and $n_{F F}$ be the number of "FF" events. For $1 \leq i \leq f_{1}$, let the weight of $i$, wt $(i)$ equal the number of "S" up to position $j$ in $A_{i}$ plus the number of "F" at or 
after $i+1$ less 1 . Theorem 1.36 will explain the criteria for constructing a SF-array.

Theorem 1.36. [8] Let $A$ be a SF-array in a tight $C S C C D(v, k, 2, b)$.

1. For any $1 \leq i \leq f_{1}, A_{i}$ contains at most one " $S$ " and at most one " $F$ ".

2. Let $A_{i}$ contain " $F$ " and the next " $S$ " appears in $A_{j}$ then $j \geq i+b-$ $2 f_{1}+1$.

3. Any three consecutive positions, $A_{i}, A_{i+1}, A_{i+2}$, may not all contain an $S$. Any three consecutive positions may not all contain an $F$.

4. There is an $S$ in $A_{1}, F$ in $A_{f_{1}}$ and $n_{S}+n_{F}=t_{1}+1$.

5. For each $1 \leq i \leq f_{1}, w t(i) \leq k$.

6. $1 \leq t_{1} \leq 2 k-1$.

7. For any $1 \leq i \leq f_{1}-1$, if $w t(i)=k$ then $w t(i+1) \neq k$.

8. $n_{S S} \leq\left\lfloor\frac{k}{2}\right\rfloor, n_{F F} \leq\left\lfloor\frac{k}{2}\right\rfloor$ and $n_{S S}+n_{F F} \leq k-1$.

9. $t_{1}+1-\min \left\{t_{1}, k\right\} \leq n_{S}, n_{F} \leq \min \left\{t_{1}, k\right\}$.

Let $\mathcal{A}$ be all the possible $\mathrm{SF}$-arrays for a tight $\operatorname{SCCD}(v, k, 2, b)$. An array $A^{\prime}$ of length $b$ with $t$ "S" and "F" is a SF-skeleton if each $S \in A^{\prime}$ is the start of an SF-array $A \in \mathcal{A}$.

SF-skeletons allow us to use necessary conditions imposed by element introductions and exits to significantly reduce work while constructing tight circular SCCD. McSorley uses SF-skeletons to enumerate all possible tight $\operatorname{CSCCD}(9,4,2,12)$ and tight $\operatorname{CSCCD}(10,4,2,15)$.

For example, consider all eight possible SF-arrays for the tight circular $\operatorname{SCCD}(9,4,2,12)$ given in Table 1.13. Each column is a SF-array based on the element $x \in T_{1}$. 


\begin{tabular}{l||l|l|l|l|l|l|l|l}
$\mathrm{R}_{x, 1}$ & $x \mathrm{SF}$ & $x \mathrm{SF}$ & $x \mathrm{SF}$ & $x \mathrm{~S}$ & $x \mathrm{SF}$ & $x \mathrm{SF}$ & $x \mathrm{SF}$ & $x \mathrm{~S}$ \\
$\mathrm{R}_{x, 2}$ & $x \mathrm{~F}$ & $x-$ & $x-$ & $x \mathrm{SF}$ & $x-$ & $x-$ & $x \mathrm{~F}$ & $x \mathrm{SF}$ \\
$\mathrm{R}_{x, 3}$ & $x-$ & $x \mathrm{SF}$ & $x \mathrm{~S}$ & $x-$ & $x \mathrm{SF}$ & $x \mathrm{SF}$ & $x-$ & $x \mathrm{~F}$ \\
$\mathrm{R}_{x, 4}$ & $x \mathrm{SF}$ & $x-$ & $x \mathrm{SF}$ & $x \mathrm{SF}$ & $x-$ & $x \mathrm{~F}$ & $x \mathrm{~S}$ & $x-$ \\
$\mathrm{R}_{x, 5}$ & $x-$ & $x \mathrm{~S}$ & $x-$ & $x-$ & $x \mathrm{SF}$ & $x-$ & $x \mathrm{SF}$ & $x \mathrm{~S}$ \\
$\mathrm{R}_{x, 6}$ & $x \mathrm{SF}$ & $x \mathrm{SF}$ & $x \mathrm{SF}$ & $x \mathrm{SF}$ & $x \mathrm{~F}$ & $x \mathrm{SF}$ & $x \mathrm{~F}$ & $x \mathrm{SF}$ \\
\hline & $(\mathrm{a})$ & (b) & $(\mathrm{c})$ & $(\mathrm{d})$ & $(\mathrm{e})$ & $(\mathrm{f})$ & $(\mathrm{g})$ & $(\mathrm{h})$
\end{tabular}

Table 1.13: SF-arrays for the tight $\operatorname{CSCCD}(9,4,2,12)$

For each SF-array we may turn this into a skeleton by appending empty rows to the array until we have the same number of rows as blocks in the design. Then for each $\mathrm{S}$ in the SF-array we place an $\mathrm{F} f_{1}-1$ rows later, in this case $f_{1}=6$. Similarly, for each $\mathrm{F}$ in the SF-array we place an $\mathrm{S}$ $f_{1}-1$ rows earlier. Lets do this for column (a) from Table 1.13 in Table 1.14. Recall that the rows are circular, so if we reach the end we wrap around to continue.

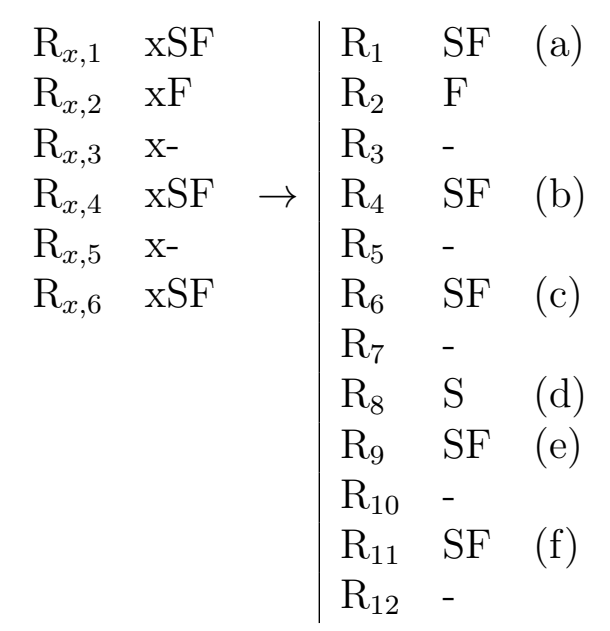

Table 1.14: skeleton for the SF-array (a) of a tight $\operatorname{CSCCD}(9,4,2,12)$

Now for each $\mathrm{S}$ in the array we need to ensure that it is the beginning of a valid SF-array, if it is not we must discard the array as it will not produce a tight CSCCD. In our example, the first $\mathrm{S}$ corresponds to array (a), the second to array (b), and so on. As each S corresponds to the start of a valid we may use this skeleton to generate a partially completed design, found in Table 1.15, where the blocks are row instead of columns. 


\begin{tabular}{l|l|llll}
$\mathrm{R}_{1}$ & $\mathrm{SF}$ & $1^{*}$ & 4 & 6 & 5 \\
\hline $\mathrm{R}_{2}$ & $\mathrm{~F}$ & 1 & & 6 & 5 \\
\hline $\mathrm{R}_{3}$ & - & 1 & & 6 & \\
\hline $\mathrm{R}_{4}$ & $\mathrm{SF}$ & 1 & $2^{*}$ & 6 & \\
\hline $\mathrm{R}_{5}$ & - & 1 & 2 & & \\
\hline $\mathrm{R}_{6}$ & $\mathrm{SF}$ & 1 & 2 & $3^{*}$ & \\
\hline $\mathrm{R}_{7}$ & - & & 2 & 3 & \\
\hline $\mathrm{R}_{8}$ & $\mathrm{~S}$ & $4^{*}$ & 2 & 3 & \\
\hline $\mathrm{R}_{9}$ & $\mathrm{SF}$ & 4 & 2 & 3 & $5^{*}$ \\
\hline $\mathrm{R}_{10}$ & - & 4 & & 3 & 5 \\
\hline $\mathrm{R}_{11}$ & $\mathrm{SF}$ & 4 & $6^{*}$ & 3 & 5 \\
\hline $\mathrm{R}_{12}$ & - & 4 & 6 & & 5
\end{tabular}

Table 1.15: Partially filled in tight $\operatorname{CSCCD}(9,4,2,12)$

From here we can complete the design much more efficiently. See Table for a completed tight $\operatorname{CSCCD}(9,4,2,12)$.

$\begin{array}{llllllllllll}\mathrm{B}_{1} & \mathrm{~B}_{2} & \mathrm{~B}_{3} & \mathrm{~B}_{4} & \mathrm{~B}_{5} & \mathrm{~B}_{6} & \mathrm{~B}_{7} & \mathrm{~B}_{8} & \mathrm{~B}_{9} & \mathrm{~B}_{10} & \mathrm{~B}_{11} & \mathrm{~B}_{12} \\ 1^{*} & 1 & 1 & 1 & 1 & 1 & 7^{*} & 4^{*} & 4 & 4 & 4 & 4 \\ 4 & 7^{*} & 7 & 2^{*} & 2 & 2 & 2 & 2 & 2 & 8^{*} & 6^{*} & 6 \\ 6 & 6 & 6 & 6 & 9^{*} & 3^{*} & 3 & 3 & 3 & 3 & 3 & 9^{*} \\ 5 & 5 & 8^{*} & 8 & 8 & 9 & 9 & 7 & 5^{*} & 5 & 5 & 5\end{array}$

Table 1.16: Completed tight $\operatorname{CSCCD}(9,4,2,12)$

We asked whether we could prove an analog of Proposition 1.24 for CSCCD. In addition to outer expansion sets we needed an additional property that allows us to avoid duplicate coverage. Suppose a $\operatorname{SCCD}(v, k, 2, b)$ has an outer expansion set that uses both $U_{0}$ and $U_{b}$. If $U_{0}=U_{1}=U_{2}=\ldots=U_{k-1}$ and $U_{b}=\bigcup_{i=1}^{k-1}\left(B_{i} \backslash B_{i+1}\right)$, then the expansion set is disjoint-capable.

For example, consider a tight $\operatorname{SCCD}(10,3,2,22)$ in Table 1.17. Here we see that $U_{0}=U_{1}+U_{2}=\{a, b\}$, and $U_{b}=\{c, d\}=B_{1} \backslash B_{2} \cup B_{2} \backslash B_{3}$. 


\begin{tabular}{cllllllllll}
$\mathrm{B}_{1}$ & $\mathrm{~B}_{2}$ & $\mathrm{~B}_{3}$ & $\mathrm{~B}_{4}$ & $\mathrm{~B}_{5}$ & $\mathrm{~B}_{6}$ & $\mathrm{~B}_{7}$ & $\mathrm{~B}_{8}$ & $\mathrm{~B}_{9}$ & $\mathrm{~B}_{10}$ & $\mathrm{~B}_{11}$ \\
$\mathrm{a}^{*}$ & $\mathrm{a}$ & $\mathrm{a}$ & $\mathrm{a}$ & $\mathrm{a}$ & $4^{*}$ & $5^{*}$ & $6^{*}$ & 6 & 6 & 6 \\
$\mathrm{~b}^{*}$ & $\mathrm{~b}$ & $\mathrm{~b}$ & $2^{*}$ & $3^{*}$ & 3 & 3 & 3 & $\mathrm{c}^{*}$ & $\mathrm{~d}^{*}$ & $\mathrm{~d}$ \\
$\wedge^{*}$ & $\mathrm{~d}^{*}$ & $1^{*}$ & 1 & 1 & 1 & 1 & $1 \wedge$ & 1 & 1 & $2^{*}$ \\
\hline
\end{tabular}

$\begin{array}{lllllllllll}\mathrm{B}_{12} & \mathrm{~B}_{13} & \mathrm{~B}_{14} & \mathrm{~B}_{15} & \mathrm{~B}_{16} & \mathrm{~B}_{17} & \mathrm{~B}_{18} & \mathrm{~B}_{19} & \mathrm{~B}_{20} & \mathrm{~B}_{21} & \mathrm{~B}_{22} \\ 6 & 6 & 6 & 6 & 4^{*} & 4 & 4 & 4 & 3^{*} & 3 & 3 \\ \mathrm{~d} & \mathrm{~d} & \mathrm{~b}^{*} & \mathrm{a}^{*} & \mathrm{a} & \mathrm{c}^{*} & 2^{*} & 2 & 2 & 2 & \mathrm{~d}^{*} \\ 4^{*} & 5^{*} & 5 & 5 & 5 \wedge & 5 & 5 & \mathrm{~b}^{*} & \mathrm{~b}_{\wedge} & \mathrm{c}^{*} & \mathrm{c}_{\wedge}\end{array}$

Table 1.17: Tight $\operatorname{SCCD}(10,3,2,22)$ [12] with a disjoint-capable expansion set.

To state our main theorem, we need a Lemma.

Lemma 1.37. Let $\left(X, \mathcal{L}=\left(B_{1}, \ldots, B_{b}\right)\right)$ be an economical $S C C D(v, k, 2, b)$. Suppose that $\left(X^{\prime}, \mathcal{M}^{\prime}=\left(B_{1}, \ldots, B_{b}, B_{b+1}^{\prime}, \ldots, B_{b^{\prime}}^{\prime}\right)\right)$ is a circular $S C C D\left(v^{\prime}, k\right.$, $\left.2, b^{\prime}\right)$ and $X \subseteq X^{\prime}$. If $B_{i}^{\prime}$ is tight $\forall i, 1 \leq i \leq b^{\prime}$, and a tight $S C C D(v, k, 2, b)$ exists in $B_{b+1}^{\prime}, \ldots, B_{b^{\prime}}^{\prime}$ then $\left(X^{\prime}, \mathcal{M}^{\prime}\right)$ is economical.

With these tools we may now state a major theorem from my undergraduate honors thesis.

Theorem 1.38. [3] If there exists a tight $\operatorname{SCCD}(v, k, 2, b)$ with a disjointcapable expansion set and a tight (economical) $S C C D\left(v^{\prime}, k, 2, b^{\prime}\right)$, then a tight (economical) circular $S C C D\left(v+v^{\prime}-2(k-1), k, 2, b^{*}\right), b^{*}=b+b^{\prime}-(k-$ $1)+\left(\frac{v}{k-1}\right)\left(v^{\prime}-2(k-1)\right)$, exists. Furthermore, if the $S C C D\left(v^{\prime}, k, 2, b^{\prime}\right)$ has an outer expansion set using both $u_{0}^{\prime}$ and $u_{b^{\prime}}^{\prime}$ then the the circular $S C C D(v+$ $\left.v^{\prime}-2(k-1), k, 2, b^{*}\right)$ has an expansion set.

Proof. Suppose that $(X, \mathcal{L})$ is a tight $S C C D(v, k, 2, b)$ with a disjoint capable outer expansion set $\mathcal{E}=\left\{u_{i_{j}}: 1 \leq j \leq \frac{v^{\prime}}{k-1}\right\}$ and $\left(X^{\prime}, \mathcal{L}^{\prime}\right)$ is an economic $S C C D\left(v^{\prime}, k, 2, b^{\prime}\right)$ with $X^{\prime}$ relabeled so $X^{\prime} \cap X=U_{0} \cup U_{b}$ and $B_{1} \supseteq U_{b}, B_{b^{\prime}} \supseteq$ $U_{0}$. To build $\left(X^{*}, \mathcal{M}^{*}\right)$, the tight circular $S C C D\left(v+v^{\prime}-2(k-1), k, 2, b^{*}\right)$, we delete the first $k-1$ blocks from $\mathcal{L}$. Next, append the blocks $\mathcal{L}^{\prime}$ to $\mathcal{L}$. Note, $\mathcal{E} \backslash\left\{U_{0} \cup U_{b}\right\}$ partitions $X \backslash X^{\prime}$. For all $x \in X^{\prime} \backslash X$ and $2 \leq j \leq \frac{v^{\prime}}{k-1}-2$, we construct $B_{i_{j}, x}^{\prime \prime}=\left(B_{i_{j}} \cap B_{i_{j}+1}\right) \cup\{x\}$ and insert $B_{i_{j}, x}^{\prime \prime}$ between $B_{i}$ and $B_{i+1}$ in any order.

The pairs in $\{x, y\}$ from $X \backslash X^{\prime}$ are covered only in $\mathcal{L}$ so are covered in $\mathcal{M}^{*}$. Similarly, the pairs $\{x, y\}$ from $X^{\prime}$ are covered in $\mathcal{L}^{\prime}$ inside $\mathcal{M}^{*}$. Since 
$\left(X^{\prime}, \mathcal{L}^{\prime}\right)$ is economical there are at most $k-1$ pairs covered more than once. $B_{i_{j}, x}^{\prime \prime}$ covers $\{x, y\}$ for all $x \in X^{\prime} \backslash X$ and $y \in X \backslash X^{\prime}$ and no other $B_{i_{j}, x}^{\prime \prime}$ covers this pair. Moreover, $U_{k}=U_{0} \subset B_{b^{\prime}}^{\prime}$ so there is a single change between the last block of the design and the first block. Therefore $\left(X^{*}, \mathcal{M}^{*}\right)$ is a circular economic SCCD.

Suppose further that $\left(X^{\prime}, \mathcal{L}^{\prime}\right)$ has expansion set $\mathcal{E}^{\prime}=\left\{U_{i_{j}}^{\prime}: 1 \leq j \leq \frac{v^{\prime}}{k-1}\right\}$. Then the economic CSCCD, $\left(X^{*}, \mathcal{M}^{*}\right)$, will have an expansion set

$$
\mathcal{E}^{*}=\left\{\left(\mathcal{E} \cup \mathcal{E}^{\prime}\right) \backslash\left\{U_{i_{0}}^{\prime}, U_{i_{b^{\prime}}}^{\prime}\right\}\right\} .
$$

For the economical version of the proof, we use the same construction and apply Lemma 1.37 .

$\begin{array}{lllllll}\mathrm{B}_{1} & \mathrm{~B}_{2} & \mathrm{~B}_{3} & \mathrm{~B}_{4} & \mathrm{~B}_{5} & \mathrm{~B}_{6} & \mathrm{~B}_{7} \\ 7^{*} & 7 & \mathrm{a}^{*} & \mathrm{~b}^{*} & \mathrm{~b} & \mathrm{~b} & \mathrm{~b} \\ \mathrm{~d} & \mathrm{~d} & \mathrm{~d} & \mathrm{~d} & \mathrm{c}^{*} & \mathrm{c} & 7^{*} \\ \mathrm{c} & 8^{*} & 8 & 8 & 8 & \mathrm{a}^{*} & \mathrm{a}\end{array}$

Table 1.18: Tight $\operatorname{SCCD}(6,3,2,7)$

\begin{tabular}{lllllllllllllllllllllll}
$\mathrm{B}_{1}$ & $\mathrm{~B}_{2}$ & $\mathrm{~B}_{3}$ & $\mathrm{~B}_{4}$ & $\mathrm{~B}_{5}$ & $\mathrm{~B}_{6}$ & $\mathrm{~B}_{7}$ & $\mathrm{~B}_{8}$ & $\mathrm{~B}_{9}$ & $\mathrm{~B}_{10}$ & $\mathrm{~B}_{11}$ & $\mathrm{~B}_{12}$ & $\mathrm{~B}_{13}$ & $\mathrm{~B}_{14}$ & $\mathrm{~B}_{15}$ & $\mathrm{~B}_{16}$ & $\mathrm{~B}_{17}$ & $\mathrm{~B}_{18}$ \\
$\mathrm{a}$ & $\mathrm{a}$ & $\mathrm{a}$ & $4^{*}$ & $5^{*}$ & $6^{*}$ & 6 & 6 & 6 & 6 & 6 & 6 & 6 & 6 & 6 & $4^{*}$ & 4 & 4 \\
$\mathrm{~b}$ & $2^{*}$ & $3^{*}$ & 3 & 3 & 3 & $7^{*}$ & $8^{*}$ & $\mathrm{c}^{*}$ & $\mathrm{~d}^{*}$ & $\mathrm{~d}$ & $\mathrm{~d}$ & $\mathrm{~d}$ & $\mathrm{~b}^{*}$ & $\mathrm{a}^{*}$ & $\mathrm{a}$ & $7^{*}$ & $8^{*}$ \\
$1^{*}$ & 1 & 1 & 1 & 1 & 1 & 1 & 1 & 1 & 1 & $2^{*}$ & $4^{*}$ & $5^{*}$ & 5 & 5 & 5 & 5 & 5 \\
\hline $\mathrm{B}_{19}$ & $\mathrm{~B}_{20}$ & $\mathrm{~B}_{21}$ & $\mathrm{~B}_{22}$ & $\mathrm{~B}_{23}$ & $\mathrm{~B}_{24}$ & $\mathrm{~B}_{25}$ & $\mathrm{~B}_{26}$ & $\mathrm{~B}_{27}$ & $\mathrm{~B}_{28}$ & $\mathrm{~B}_{29}$ & $\mathrm{~B}_{30}$ & $\mathrm{~B}_{31}$ & $\mathrm{~B}_{32}$ & $\mathrm{~B}_{33}$ & & \\
4 & 4 & 4 & $3^{*}$ & 3 & 3 & 3 & 3 & $7^{*}$ & 7 & $\mathrm{a}^{*}$ & $\mathrm{~b}^{*}$ & $\mathrm{~b}$ & $\mathrm{~b}$ & $\mathrm{~b}$ & & \\
$\mathrm{c}^{*}$ & $2^{*}$ & 2 & 2 & 2 & 2 & 2 & $d^{*}$ & $\mathrm{~d}$ & $\mathrm{~d}$ & $\mathrm{~d}$ & $\mathrm{~d}$ & $\mathrm{c}^{*}$ & $\mathrm{c}$ & $7^{*}$ & & \\
5 & 5 & $\mathrm{~b}^{*} \mathrm{~b}$ & $7^{*}$ & $8^{*}$ & $\mathrm{c}^{*}$ & $\mathrm{c}$ & $\mathrm{c}$ & $8^{*}$ & 8 & 8 & 8 & $\mathrm{a}^{*}$ & $\mathrm{a}$ & &
\end{tabular}

Table 1.19: Tight CSCCD $(12,3,2,33)$

One might think that requiring a disjoint-capable outer expansion set is limiting for building circular SCCD. However, it is possible to build a design with this property as long as you have an outer expansion set that uses both $U_{0}$ and $U_{b}$. 
Theorem 1.39. [3] Let $(X, \mathcal{L})$ be a tight $S C C D(v, k, 2, b)$ with an outer expansion set that uses both $U_{0}$ and $U_{b}$. Then there exists a tight $S C C D(2 v-$ $\left.k+1, k, 2,2 b+\frac{v}{k-1}(v-k+1)\right)$ with a disjoint-capable expansion set.

We can use the above lemmas, propositions, theorems, known SCCD, and SCCD to prove the following.

Theorem 1.40. The following single-change covering designs exist.

1. There exists a tight $\operatorname{SCCD}(v, 2,2, b)$ for all $v$. [9]

2. An economical $\operatorname{SCCD}(v, 3,2, b)$ exists for all $v \geq 6$. These are tight if $v \equiv 2,3(\bmod 4) \cdot[15]$

3. An economical $\operatorname{SCCD}(v, 4,2, b)$ exists for all $v \geq 12$. [11] These are tight if $v \equiv 0,1(\bmod 3) .[12]$

4. An economical $\operatorname{SCCD}(v, 5,2, b)$ exists for all $v \equiv 4,5,6(\bmod 16)$, $v \geq 20$. These are tight if $v \equiv 4,5(\bmod 6)[3]$

5. A tight $\operatorname{CSCCD}(v, 2,2, b)$ exists for all $v \geq 3$. [8]

6. An economical $\operatorname{CSCCD}(v, 3,2, b)$ exists for all $v \geq 4$. These are tight if and only if $v \equiv 0,1(\bmod 4) .[8]$

7. An economical $\operatorname{CSCCD}(v, 4,2, b)$ exists for all $v \geq 27$ [3]. These are tight if and only if $v \equiv 0,1(\bmod 3)$.

8. An economical $\operatorname{CSCCD}(v, 5,2, b)$ exists for all $v \equiv 0,1,2(\bmod 16)$, $v \geq 480$. These are tight if $v \equiv 0,1(\bmod 16)$. [3]

Proof. 1. (1) Nelder gives the construction. [9]

2. A tight $\operatorname{SCCD}(6,3,2,7)$ (Table 1.18) exists. An economic $\operatorname{SCCD}(8,3,2,14)$ exists. Using a tight $\operatorname{SCCD}(6,3,2,7)$ and Theorem 1.24 we form a tight $\operatorname{SCCD}(7,3,2,10)$. Using economic $\operatorname{SCCD}(8,3,2,14)$ and Theorem 1.24 we form an economic $\operatorname{SCCD}(9,3,2,18)$. By Lemma 1.6 and tight $\operatorname{SCCD}(6,3,2,7)$, tight $\operatorname{SCCD}(7,3,2,10)$, economical $\operatorname{SCCD}(8,3,2,14)$, economical $\operatorname{SCCD}(9,3,2,18)$ we build tight $\operatorname{SCCD}(10,3,2,24)$, tight $\operatorname{SCCD}(11,3,2,27)$, economic $\operatorname{SCCD}(12,3,2,33)$, and economical SCCD $(13,3,2,39)$ respectively. Suppose we form each tight $\operatorname{SCCD}(6+4 i, 3,2$, $\left.7+\sum_{j=0}^{i}(2(4 j+6)+3)\right)$, tight $\operatorname{SCCD}\left(7+4 i, 3,2,10+\sum_{j=0}^{i}(2(4 j+\right.$ 
$7)+3)$ ), economic $\operatorname{SCCD}\left(8+4 i, 3,2,14+\sum_{j=0}^{i}(2(4 j+8)+3)\right)$, and economic $\operatorname{SCCD}\left(9+4 i, 3,2,18+\sum_{j=0}^{i}(2(4 j+9)+3)\right)$ this way and this holds for each $i=n \geq 1$, when $i=n+1$ we have

$$
\begin{aligned}
7+\sum_{j=0}^{n+1}(2(4 j+6)+3)=7 & +\sum_{j=0}^{n}(2(4 j+6)+3) \\
& +2(4(n+1)+6)+3 \\
10+\sum_{j=0}^{n+1}(2(4 j+7)+3)=10 & +\sum_{j=0}^{n}(2(4 j+7)+3) \\
& +2(4(n+1)+7)+3 \\
14+\sum_{j=0}^{n+1}(2(4 j+8)+3)=14 & +\sum_{j=0}^{n}(2(4 j+8)+3) \\
& +2(4(n+1)+8)+3 \\
18+\sum_{j=0}^{n+1}(2(4 j+9)+3)=18 & +\sum_{j=0}^{n}(2(4 j+9)+3) \\
& +2(4(n+1)+9)+3
\end{aligned}
$$

3. Similarly to (2), there exists a tight $\operatorname{SCCD}(12,4,2,21)$, a tight $\operatorname{SCCD}(15,4,2,34)$, and a tight $\operatorname{SCCD}(18,4,2,50)$ with expansion sets. Using the tight $\operatorname{SCCD}(12,4,2,21)$ and a tight $\operatorname{SCCD}(12,4,2,21)$, a tight $\operatorname{SCCD}(15,4,2,34)$, or a tight $\operatorname{SCCD}(18,4,2,50)$ respectively with Theorem 1.26 we may construct the tight $\operatorname{SCCD}(21,4,2,69)$, the tight $\operatorname{SCCD}(24,4,2,91)$, and the tight $\operatorname{SCCD}(27,4,2,116)$. We ultimately obtain all the tight $\operatorname{SCCD}(v, 4,2, b)$ with $v \equiv 0,3,6(\bmod 9)$. So we have a construction for all the tight $\operatorname{SCCD}(v, 4,2, b)$ where $v \equiv 0(\bmod 3)$. Using these designs which all have expansion sets and Theorem 1.24 or Theorem 1.25 we obtain the tight $\operatorname{SCCD}(v, 4,2, b)$ with $v \equiv 1(\bmod 3)$ and $v \equiv 2(\bmod 3)$ respectively, as desired.

4. Similarly to (2) and (3), a tight $\operatorname{SCCD}(20,5,2,46)$ exists with an expansion set. We recursively apply the results of Theorem 1.26 and a tight $\operatorname{SCCD}(20,5,2,46)$ to find all tight $\operatorname{SCCD}(v, 5,2, b)$ with $v \equiv 4$ $(\bmod 16)$. From here we apply Proposition 1.24 to obtain the tight 
$\operatorname{SCCD}(v, 5,2, b)$ with $v \equiv 5(\bmod 16)$. We use the tight $\operatorname{SCCD}(v, 5,2, b)$ with $v \equiv 4(\bmod 16)$ obtained in $(4)$ and Theorem 1.25 to build the economic $\operatorname{SCCD}(v, 5,2, b)$ with $v \equiv 6(\bmod 16)$.

5. This follows from McSorley's Theorem 1.32.

6. This follows from McSorley's Theorem 1.32.

7. If a tight circular $\operatorname{SCCD}(v, 4,2, b)$ exists then $b=\frac{v(v-1)}{6} \in \mathbb{N}$, so $v \equiv 0,1(\bmod 3)$. From $(3)$ for all $v \geq 12, v \equiv 0,1(\bmod 3)$ there exits a $\operatorname{SCCD}(v, 4,2, b)$. Using a tight $\operatorname{SCCD}(21,4,2,69)$ with a disjointcapable expansion set and Theorem 1.38 we can construct a circular tight $\operatorname{SCCD}(v+15,4,2, b)$ therefore we can construct a circular tight $\operatorname{SCCD}(v, 4,2, b)$ for every $v \geq 27, v \equiv 0,1(\bmod 3)$. From (3) we have that for all $v \geq 14, v \equiv 2(\bmod 3)$ there exits an economic $\operatorname{SCCD}(v, 4,2, b)$. Using the tight $\operatorname{SCCD}(21,4,2,69)$ with a disjointcapable expansion set and Theorem 1.38 we can construct an economical circular $\operatorname{SCCD}(v+15,4,2, b)$ therefore we can construct a tight circular $\operatorname{SCCD}(v, 4,2, b)$ for every $v \geq 29, v \equiv 2(\bmod 3)$.

8. Similarly, we use two tight $\operatorname{SCCD}(20,5,2,46)$ to build a tight $\operatorname{SCCD}(36,5,2,156)$ with a disjoint capable outer expansion set and apply the same Theorems and Propositions to construct the tight $\operatorname{CSCCD}(v, 5,2, b)$ for all $v \equiv 0,1(\bmod 16)$. Similarly, we use the tight $\operatorname{SCCD}(36,5,2,156)$ from $(9)$ and the economical $\operatorname{SCCD}(v, 5,2, b)$ from (5) to construct economical $\operatorname{CSCCD}(v, 5,2, b)$ for all $v \equiv 2(\bmod 16), v \geq 50$ 


\section{Chapter 2}

\section{Single Change Covering Designs}

We will begin by considering a new construction.

Theorem 2.1. Let $(X, \mathcal{L})$ be a $\operatorname{SCCD}(v, 3,2, b)$ containing three consecutive blocks of the form

$\begin{array}{lll}B_{i} & B_{i+1} & B_{i+2} \\ 2 & 3^{*} & 3 \\ 1 & 1 & 4^{*} \\ 0 & 0 & 0\end{array}$

Table 2.1: Consecutive blocks to replace

Then there exists a $S C C D(v+4,3,2, b+2 v+3),\left(X^{\prime}, \mathcal{L}^{\prime}\right)$, containing three consecutive blocks of the form given in Table 2.1. Further, if $(X, \mathcal{L})$ is circular, economical, tight or contains an expansion set then so does $\left(X^{\prime}, \mathcal{L}^{\prime}\right)$.

Proof. Let $(X, \mathcal{L})$ be a $\operatorname{SCCD}(v, 3,2, b)$ containing $B_{i}, B_{i+1}$, and $B_{i+2}$ as proposed. Let $\{a, b, c, d\}$ be disjoint from $X$ and $X^{\prime}=X \cup\{a, b, c, d\}$. To construct $\mathcal{L}^{\prime}$ we first replace blocks $B_{i}, B_{i+1}$ and $B_{i+2}$ from $\mathcal{L}$ with the following fourteen blocks, $\mathcal{N}$. 


$\begin{array}{lllllllllllllll}\mathrm{N}_{1} & \mathrm{~N}_{2} & \mathrm{~N}_{3} & \mathrm{~N}_{4} & \mathrm{~N}_{5} & \mathrm{~N}_{6} & \mathrm{~N}_{7} & \mathrm{~N}_{8} & \mathrm{~N}_{9} & \mathrm{~N}_{10} & \mathrm{~N}_{11} & \mathrm{~N}_{12} & \mathrm{~N}_{13} & \mathrm{~N}_{14} \\ 2 & d^{*} & a^{*} & a & a & 3^{*} & 3 & 3 & 2^{*} & 2 & 2 & 0^{*} & 0 & 0 \\ 1 & 1 & 1 & 1 & 1 & 1 & d^{*} & d & d & d & c^{*} & c & 3^{*} & 3 \\ 0 & 0 & 0 & b^{*} & c^{*} & c & c & a_{\wedge}^{*} & a & b^{*} & b_{\wedge} & b & b & 4^{*}\end{array}$

Table 2.2: $\mathcal{N}$

Note that $B_{i}=N_{1}$ and $B_{i+2}=N_{14}$ and the sequence $\mathcal{N}$ is single change so the new sequence of blocks remain a single change ordering. The sequence $\mathcal{N}$ covers exactly the same pairs from $\{0,1,2,3\}$ as $B_{i}, B_{i+1}$ and $B_{i+2}$ did. Further, $\mathcal{N}$ covers all the pairs in $\{a, b, c, d\}$ as well as all the pairs between $\{0,1,2,3\}$ and $\{a, b, c, d\}$. Thus, the only pairs not covered yet are those between $\{a, b, c, d\}$ and $X \backslash\{0,1,2,3\}$. By using the expansion set on the points $\{\mathrm{a}, \mathrm{b}, \mathrm{c}, \mathrm{d}\}$ in $\mathcal{N}$ at expansion locations $u_{8}$ and $u_{11}$ we insert the blocks $\{a, d\} \cup\{x\}$ and $\{b, c\} \cup\{x\}$ for $x \in X \backslash\{0,1,2,3\}$ respectively. Thus, $\left(X^{\prime}, \mathcal{L}^{\prime}\right)$ is a $\operatorname{SCCD}(v+4,3,2, b+2 v+3)$. Moreover, the blocks colored blue in Table 2.2 have the form given in Table 2.1 and no blocks are inserted between these, so $(X, \mathcal{L})$ has the form specified by Table 2.1 .

As the first and last blocks of $\left(X^{\prime}, \mathcal{L}^{\prime}\right)$ remain unchanged from $(X, \mathcal{L})$ the design remains circular if it began as circular. As

$$
\frac{\left(\begin{array}{c}
v+4 \\
2
\end{array}\right)-\left(\begin{array}{l}
v \\
2
\end{array}\right)}{k-1}=\frac{\left(\begin{array}{c}
v+4 \\
2
\end{array}\right)-\left(\begin{array}{l}
v \\
2
\end{array}\right)}{3-1}=2 v+3,
$$

if $(X, \mathcal{L})$ is tight or economical then $\left(X^{\prime}, \mathcal{L}^{\prime}\right)$ is tight or economical respectively. Finally, we note that the set of unchanged subsets of $\left(X^{\prime}, \mathcal{L}^{\prime}\right)$ is the union of the set of unchanged subsets from $(X, \mathcal{L})$ and the set of unchanged subsets from $\mathcal{N}$, which contains $\{a, d\}$ and $\{b, c\}$. Therefore, if $(X, \mathcal{L})$ contained an expansion set, then so does $\left(X^{\prime}, \mathcal{L}^{\prime}\right)$.

For example we consider the tight $\operatorname{SCCD}(6,3,2,7)$ in Table 2.3. Blocks $B_{3}, B_{4}, B_{5}$ have the required form. Table 2.4 shows the blocks after $B_{3}, B_{4}, B_{5}$ are replaced. Table 2.5 shows the tight $\operatorname{SCCD}(10,3,2,22)$.

$\begin{array}{cllllll}\text { B1 } & \text { B2 } & \text { B3 } & \text { B4 } & \text { B5 } & \text { B6 } & \text { B7 } \\ 5^{*} & 5 & 2^{*} & 3^{*} & 3 & 3 & 3 \\ 1^{*} & 1 & 1 & 1 & 4^{*} & 4 & 5^{*} \\ 4^{*} & 0^{*} \wedge & 0 & 0 & 0 & 2^{*} \wedge & 2\end{array}$

Table 2.3: Tight $\operatorname{SCCD}(6,3,2,7)$ 


\begin{tabular}{clllllllll}
$\mathrm{B} 1$ & $\mathrm{~B} 2$ & $\mathrm{~B} 3$ & $\mathrm{~B} 4$ & $\mathrm{~B} 5$ & $\mathrm{~B} 6$ & $\mathrm{~B} 7$ & $\mathrm{~B} 8$ & $\mathrm{~B} 9$ & $\mathrm{~B} 10$ \\
$5^{*}$ & 5 & $2^{*}$ & $d^{*}$ & $a^{*}$ & $a$ & $a$ & $3^{*}$ & 3 & 3 \\
$1^{*}$ & 1 & 1 & 1 & 1 & 1 & 1 & 1 & $d^{*}$ & $d$ \\
$4^{*}$ & $0^{*} \wedge$ & 0 & 0 & 0 & $b^{*}$ & $c^{*}$ & $c$ & $c$ & $a_{\wedge}^{*}$ \\
\hline $\mathrm{B} 11$ & $\mathrm{~B} 12$ & $\mathrm{~B} 13$ & $\mathrm{~B} 14$ & $\mathrm{~B} 15$ & $\mathrm{~B} 16$ & $\mathrm{~B} 17$ & $\mathrm{~B} 18$ & & \\
$2^{*}$ & 2 & 2 & $0^{*}$ & 0 & 0 & $2^{*}$ & 2 & & \\
$d$ & $d$ & $c^{*}$ & $c$ & $3^{*}$ & 3 & 3 & 3 & & \\
$a$ & $b^{*}$ & $b_{\wedge}$ & $b$ & $b$ & $4^{*}$ & $4 \wedge$ & $5^{*}$ & &
\end{tabular}

Table 2.4: The extended tight $\operatorname{SCCD}(6,3,2,7)$ with the 14 inserted blocks

\begin{tabular}{cllllllllll} 
B1 & B2 & B3 & B4 & B5 & B6 & B7 & B8 & B9 & B10 & B11 \\
$5^{*}$ & 5 & $2^{*}$ & $d^{*}$ & $a^{*}$ & $a$ & $a$ & $3^{*}$ & 3 & 3 & $4^{*}$ \\
$1^{*}$ & 1 & 1 & 1 & 1 & 1 & 1 & 1 & $d^{*}$ & $d$ & $d$ \\
$4^{*}$ & $0^{*} \wedge$ & 0 & 0 & 0 & $b^{*}$ & $c^{*}$ & $c$ & $c$ & $a_{\wedge}^{*}$ & $a$ \\
\hline $\mathrm{B} 12$ & $\mathrm{~B} 13$ & $\mathrm{~B} 14$ & $\mathrm{~B} 15$ & $\mathrm{~B} 16$ & $\mathrm{~B} 17$ & $\mathrm{~B} 18$ & $\mathrm{~B} 19$ & $\mathrm{~B} 20$ & $\mathrm{~B} 21$ & $\mathrm{~B} 22$ \\
$5^{*}$ & $2^{*}$ & 2 & 2 & $4^{*}$ & $5^{*}$ & $0^{*}$ & 0 & 0 & $2^{*}$ & 2 \\
$d$ & $d$ & $d$ & $c^{*}$ & $c$ & $c$ & $c$ & $3^{*}$ & 3 & 3 & 3 \\
$a$ & $a$ & $b^{*}$ & $b_{\wedge}$ & $b$ & $b$ & $b$ & $b$ & $4^{*}$ & $4 \wedge$ & $5^{*}$
\end{tabular}

Table 2.5: The completed tight $\operatorname{SCCD}(10,3,2,22)$ based on the tight $\operatorname{SCCD}(6,3,2,7)$

The three consecutive blocks isomorphic to those in Table 2.1 are highlighted in blue. We can repeat this process on each newly constructed design to get all designs of the form $(6+4 i, 3,2, b+2(v+4(i-1))+3), i \geq 0$. Further, each of these tight $\operatorname{SCCD}(6+4 i, 3,2, b+2(v+4(i-1))+3)$ has an expansion set, therefore we construct tight $S C C D\left(7+4 i, 3,2, b+2(v+4(i-1))+3+\frac{v}{2}\right)$ by Proposition 1.24. Thus, this construction provides to an alternate proof on the existence of $\operatorname{SCCD}(v, 3,2, b)$ and $\operatorname{CSCCD}(v, 3,2, b)$ in Theorem 3.

\subsection{Efficient Computational Search Implementation}

I adapted Phillips efficiencies in Lemmas 1.29, 1.30, and 1.31 to a search for tight CSCCD using SF-arrays. 
After we generate a skeleton, we can partially fill in the CSCCD with all elements of $T_{1}$. We will choose the block that contains the largest number of elements from $T_{1}$ to be $B_{1}$. In a circular SCCD a cyclic shift can be used to make any block the first [8]. If we limit early choice, we reduce the exponential growth of a search tree. In the event that two blocks are partially filled equally, we use the block that initiates the longest run of initially filled blocks. We call this front loading the design.

We say that two CSCCD are equivalent if one is obtained from permuting the symbol set $X$, or cyclically shifting $\mathcal{L}$ or reversing $\mathcal{L}$ in our search ensures we fix $T_{1}=\left\{0,1, \ldots, t_{1}-1\right\}, T_{2}=\left\{t_{1}, t_{1}+1, \ldots, t_{1}+t_{2}-1\right\}, \ldots, T_{M}=$ $\left\{\sum_{j=1}^{M-1} t_{j}, \ldots, v\right\}$. Further, we assume

Assumption 2.2. For each $x_{i}, x_{j} \in T_{n}, x_{i}$ must be introduced for the first time before $x_{j}$ if $x_{i}<x_{j}$.

Now we consider the structure of the SF-arrays in the skeleton.

Observation 2.3. Suppose there exists a partial construction of a circular $\operatorname{SCCD}(v, k, 2, b)$ up until block $B_{i}$. If $x$ 's $\mathrm{F}$ is in block $B_{i}$ in the skeleton, then all other elements of $B_{i}$ are also in $B_{i+1}$.

Observation 2.4. Suppose there exists a partial construction of a circular $\operatorname{SCCD}(v, k, 2, b)$ up until block $B_{i}$. If $x$ 's $\mathrm{F}$ is in block $B_{i}$ in the skeleton, then all other element of $B_{i}$ are in $B_{i-1}$.

Observation 2.5. If block $B_{i}$ contains an "S" and "F" both observations apply.

In a tight CSCCD Phillip's notion of minor variants remains unchanged, and we use Lemma 1.29 exactly as he did to optimize our search.

However, when we consider Phillip's end permutations, we must now account for the circular property.

Lemma 2.6. If there exists a $\operatorname{CSCCD}(v, k, 2, b)$ and in blocks $B_{1}, \ldots, B_{i}$, $\{x, z\},\{y, z\}, z \in Z$ are exactly the pairs counting $x, y$ and either $x, y \in B_{0}$ or $x, y \notin B_{0}$. Then swapping $x, y$ in blocks $B_{i+1}, \ldots, B_{b}$ produces a circular $S C C D(v, k, 2, b)$.

Proof. Let $(X, \mathcal{L})$ be a $\operatorname{CSCCD}(v, k, 2, b)$. Suppose elements $x, y \in X$ cover the pairs $\{x, z\},\{y, z\}, \forall z \in Y \subseteq X$ in blocks $B_{1}, \ldots, B_{i}$. If $x, y \in B_{0}$ then $x, y$ still need to cover the pairs $\{x, w\},\{y, w\} \forall w \in W \subset X \backslash Y$. The blocks 
$B_{i+1}, \ldots, B_{b}$ would cover each of these pairs. So swapping $x$ and $y$ 's introductions will produce a $\operatorname{CSCCD}(v, k, 2, b)$ which will cover the pairs $\{x, w\}$ where $\{y, w\}$ was covered and $\{y, w\}$ where $\{x, w\}$ was covered. We have not effected the single change property either, so $(X, \mathcal{L})$ is still a $\operatorname{CSCCD}(v, k, 2, b)$. We do similarly for $x, y \notin B_{0}$. If $x \in B_{0}$ and $y \notin B_{0}$ we may loose the single change between $B_{0}$ and $B_{b}$.

In tight $\operatorname{CSCCD}(v, k, 2, b)$ if $x \in B_{i}$ and there are $0<m<(k-1)$ pairs containing $x$ not covered in $B_{0}, \ldots, B_{i}$ then $x \in B_{i+1}$. We use this the same way as Phillips: if the conditions hold in a partially computed CSCCD we only continue the search if $x$ next introduction after $B_{i}$ is feasible.

We may also consider the removal conditions of Phillips in Lemma 1.31 with a minor modification for circular. Namely, in block $B_{b}$ we do not consider Lemma 1.31 as we would need to account for the elements in $B_{1}$. In our search we do not remove such an $x$ from $B_{i}$, with the exception in block $B_{b}$.

Conversely, in tight $\operatorname{CSCCD}(v, k, 2, b)$ for $x \in B_{j}$ suppose that $x \in T_{i}$ and has been introduced $i-p$ times and all but $m$ pairs containing $x$ are covered in $B_{1}, \ldots, B_{j}$. Then $x$ will be in exactly $h=m-p(k-2)$ more blocks, which leads us to the following lemma. Let $\tilde{\mathcal{L}}$ be a partially completed design up to $B_{j}, B_{j} \in \tilde{\mathcal{L}}$. Let $\tilde{f}_{x}$ be the frequency of $x \in \tilde{\mathcal{L}}$. We say this is a persistence condition.

Lemma 2.7. If $f_{x}<\tilde{f}_{x}+m-p(k-2)$ then $x$ must be removed in $B_{i}$.

Proof. If $f_{x}<\tilde{f}_{x}+m-p(k-2)$ then we will be forced to cover a pair a second time during an introduction, so $x$ must leave.

\section{$2.2 \quad$ Algorithms}

Using these Lemmas in conjunction we significantly speed up the search.

The algorithm starts by generating all valid SF arrays by a backtracking search using the conditions outlined in Theorem 1.36. Then we generate the skeletons by extending the SF-arrays. We place an " $\mathrm{F}$ " $f_{1}$ blocks after each "S" of the SF-array and an "S" $f_{1}$ blocks before each "F" of the SF-array. We need to validate the skeletons generated by confirming that each " $\mathrm{S}$ " in the skeleton is the beginning of a valid SF-array. We eliminate all cyclic shifts so all skeletons are non-isomorphic. For each skeleton we pick the cyclic first block such that $B_{1}$ will contain as many elements $T_{1}$ as possible. 
Second, let $\mathcal{L}$ be a $k \times b$ array, each column is a block and rows are positions within a block. Now we fill in the blocks with the elements of $T_{1}$ starting with 0 . To do so, we introduce the elements in increasing row order. Let $j$ be the first block where a point from $B_{1}$ is removed. All "S" before $B_{j}$ are filled in with the next available point from $T_{1}$ in the next available position. In block $B_{j}$ or after, "S" must be replaced by an element in each possible position. Each of the resulting partially filled in skeletons is our potential block list, $\mathcal{L}$.

Third, now fill in $\mathcal{L}$ with a recursive search, Algorithm 2.2.2. The Recursive Search first checks if $\mathcal{L}$ is filled in. If so, we check that the design satisfies the end conditions. That is, we check if we are on $B_{b}$, if $\left|B_{b}\right|=k$, and if $\left|B_{b} \cap B_{1}\right|=k-1$. Second, it checks if we are introducing an element on the first block. If we are, we call Update First Block. Now Recursive Search checks if the point introduced in this block, $B_{i}$, is forced by the skeleton. If it is, then we say that $x$ is forced. Further, if $x$ is not listed as an introduction, Recursive Search checks that $x$ is valid by checking if the pairs $\{x, y\}, \forall y \in B_{i-1}$ not in the same position as $x$. After we determine $x$ is valid in this situation we call Update No New Points. Finally, $x \in B_{i}$ is not an element from $T_{1}$, so we consider all possible points to introduce in increasing order. If we are not on the first block, suppose that $x \in T_{j}$ then we know that it will be introduced $j$ times and appears in $f_{j}$ blocks. We check that introducing $x$ in $B_{i}$ does not exceed $j$ or $f_{j}$ nor violates the assumptions of 2.2. If there exists a $y<x$ such that $x$ and $y$ satisfy the conditions of Lemma 2.6 we do not consider introducing $x$. If conditions allow we call Update. Otherwise, if we are not on $B_{1}$, Recursive Search checks that introducing $x$ in $B_{i}$ does not violate the assumptions of 2.2 and calls Update First Block if conditions allow.

Update, Algorithm 2.2.3, does the following for every empty row position in $B_{i}$. Update ensures we are using the smallest minor variant as in Lemma 1.29. Now we need to check for every $y$ in $B_{i-1}$ that does not share a row position with $x$ such that no pairs $\{x, y\}$ were previously covered. This is $U_{i-1}$. The algorithm also checks that placing $x$ and $U_{i-1}$ in $B_{i}$ will respect Observations 2.3, 2.4, and 2.5. Now we check that introducing $x$ in $B_{i}$ does not exceed $j$ or $f_{j}$ and that each $y \in U_{i-1}$, where $y \in T_{w} y$, does not exceed $f_{w}$. Update checks if $x$ must be in the next block as in Lemma 1.31 or if $x$ must be removed as in Lemma 2.7 and that we can do these. When all these checks are passed, $\forall y \in U_{i-1}$ add $y$ to $B_{i}$ in the same row position and add $x$ to $B_{i}$. Now increment the frequency count of each element in the block and 
the introduction count for $x$ and add all the pairs covered. Call Recursive $\operatorname{Search}(\mathcal{L}, i+1)$. When the search has returned, decrements frequency and introduction counts, remove covered pairs and remove $x$ and the elements of $U_{i-1}$ that were added from $B_{i-1}$.

Update No New Points, Algorithm 2.2.4, ensures we are using the smallest minor variant as in Lemma 1.29. Now we check that the introduced element $x$ and elements of the unchanged subset that were previously forced forward by Observations 2.3, 2.4, and 2.5 are not covered more then once. Now we bring any elements from $B_{i-1}$ not already in $B_{i}$ to $B_{i}$. Now we add $x$ to $\mathcal{L}$ and add the pairs covered and introduction and frequency counts to $\mathcal{L}$. Call Recursive $\operatorname{Search}(\mathcal{L}, i+1)$. Now we remove the pairs covered, decrements the introductions of $x$, decrements the frequency count for every element in $B_{i}$. Remove $x$ and the elements we brought froward from $B_{i-1}$.

Update First Block, Algorithm 2.2.5, does the following for each empty row position in $B_{1}$. Increment the frequency count and add the new pair that is covered to $\mathcal{L}$. If the block is now complete call Recursive $\operatorname{Search}(\mathcal{L}, 2)$ starting on the next block, otherwise call Recursive $\operatorname{Search}(\mathcal{L}, 1)$ on the first block. When Recursive Search returns we decrease the frequency count and remove the pair that was covered from $\mathcal{L}$.

Update First Block No New Points, Algorithm 2.2.6, adds $x$ to $\mathcal{L}$ and increases the introduction and frequency count and save the new covered pairs. If the block is now complete it calls Recursive $\operatorname{Search}(\mathcal{L}, 2)$ starting on the next block, otherwise it calls Recursive $\operatorname{Search}(\mathcal{L}, 1)$ on the first block. When Recursive Search returns, decrease the introduction and frequency count and remove the pairs that were covered.

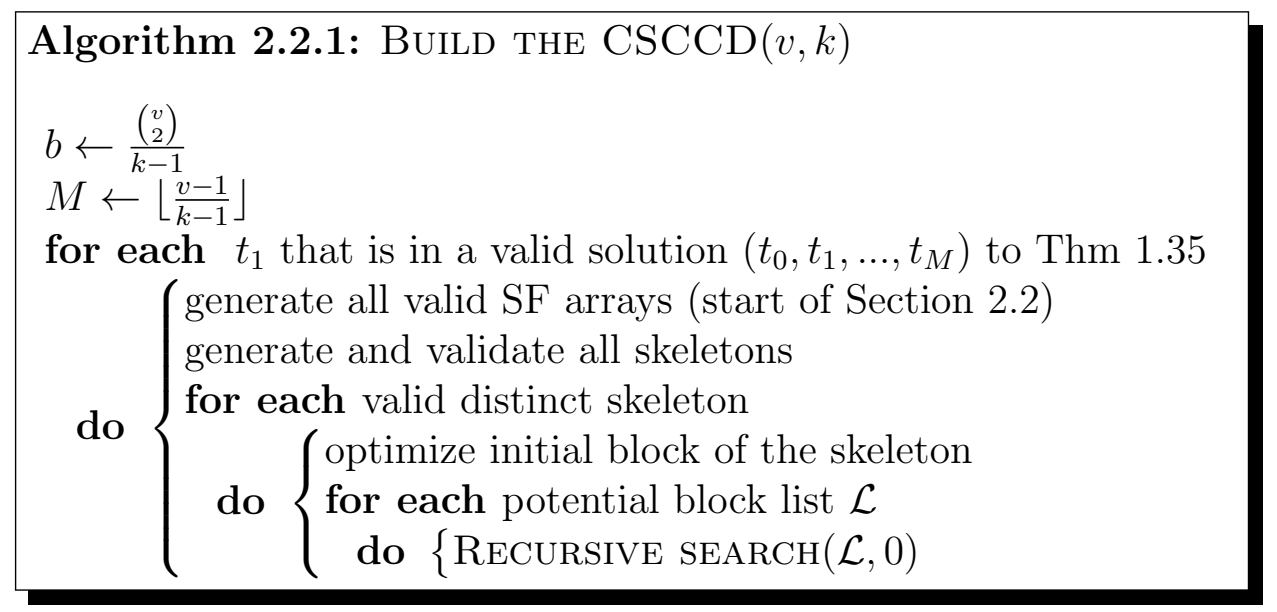




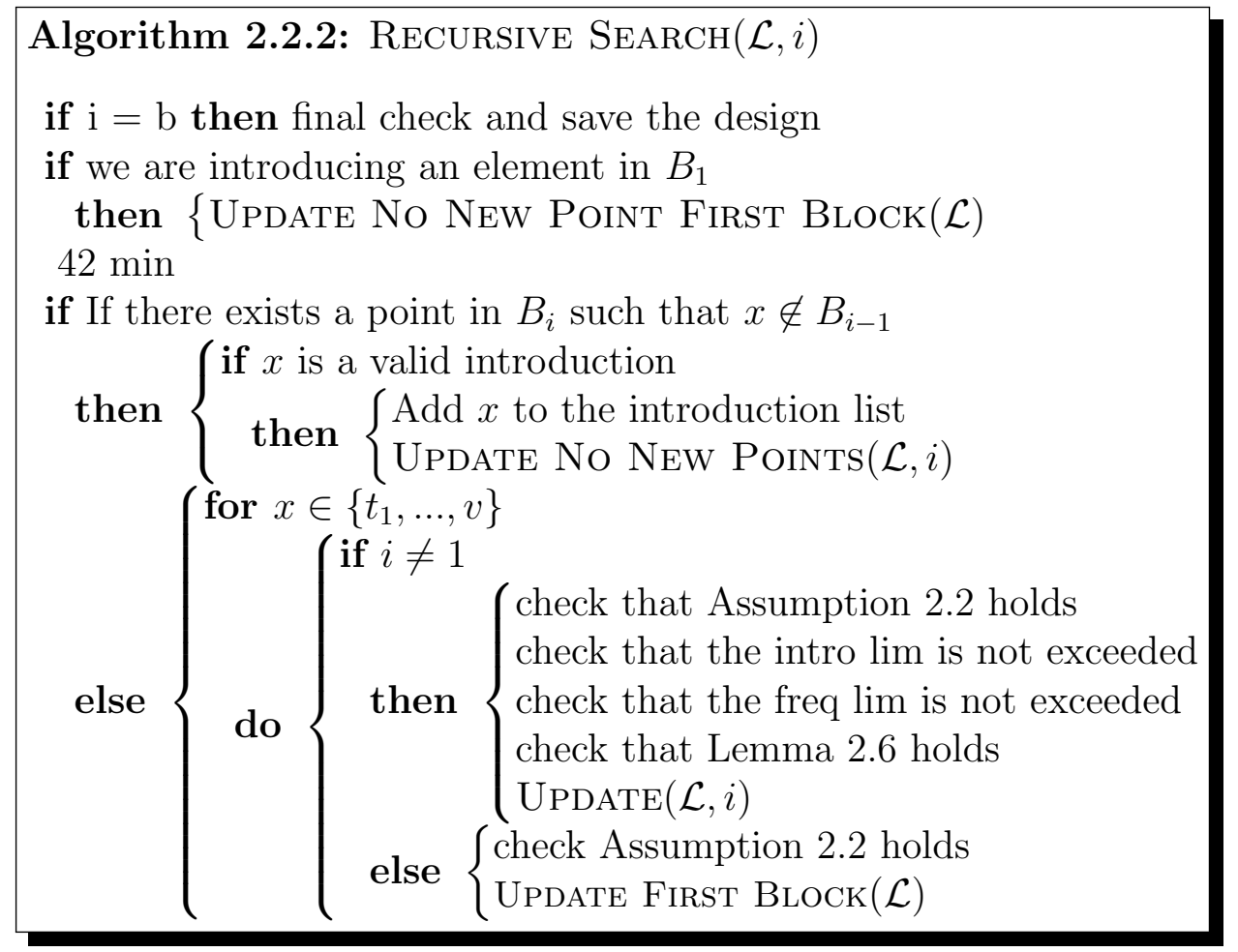




\begin{tabular}{l} 
Algorithm 2.2.3: $\operatorname{UpDATE}(\mathcal{L}, i)$ \\
for each for each valid introduction position \\
$\mathbf{d o}\left\{\begin{array}{l}\text { check that Lemma } 1.29 \text { holds } \\
\text { check that } U_{i-1} \text { does not cover pairs already covered } \\
\text { check that } U_{i-1} \text { and } x \text { sat Observations } 2.3,2.4, \text { and } 2.5 \\
\text { check that the intro limit of } x \text { is not exceeded } \\
\text { check that the frequency limit of } U_{i-1} \text { is not exceeded } \\
\text { check that Lemma } 1.31 \text { holds } \\
\text { check that Lemma } 2.7 \text { holds } \\
\text { add elem from } U_{i-1} \text { to } B_{i} \text { and future blocks when required } \\
\text { add pairs covered } \\
\text { increment number of introduction and frequency } x \\
\text { increment frequency count for } y \in U_{i-1} \\
\text { Recursive SEARCH }(\mathcal{L}, i+1) \\
\text { remove pairs covered } \\
\text { decrements number of introductions and frequency for } x \\
\text { decrements frequency count for } y \in U_{i-1} \\
\text { remove elements added to relevant blocks from } U_{i-1}\end{array}\right.$ \\
\hline
\end{tabular}

\footnotetext{
Algorithm 2.2.4: $\operatorname{Update}$ No New $\operatorname{Points}(\mathcal{L}, i)$

check that Lemma 1.29 holds

check that elements forced by Observations 2.3, 2.4, and 2.5 hold add elements from $U_{i-1}$ to $B_{i}$ and future blocks when required increment number of introduction and frequency of $x$ increment number of introductions of unchanged subset add pairs covered ReCursive $\operatorname{Search}(\mathcal{L}, i+1)$ remove the pairs covered decrements number of introductions and frequency for $x$ decrements frequency for elements of $U_{i-1}$ removed elements added to relevant blocks from $U_{i-1}$
} 


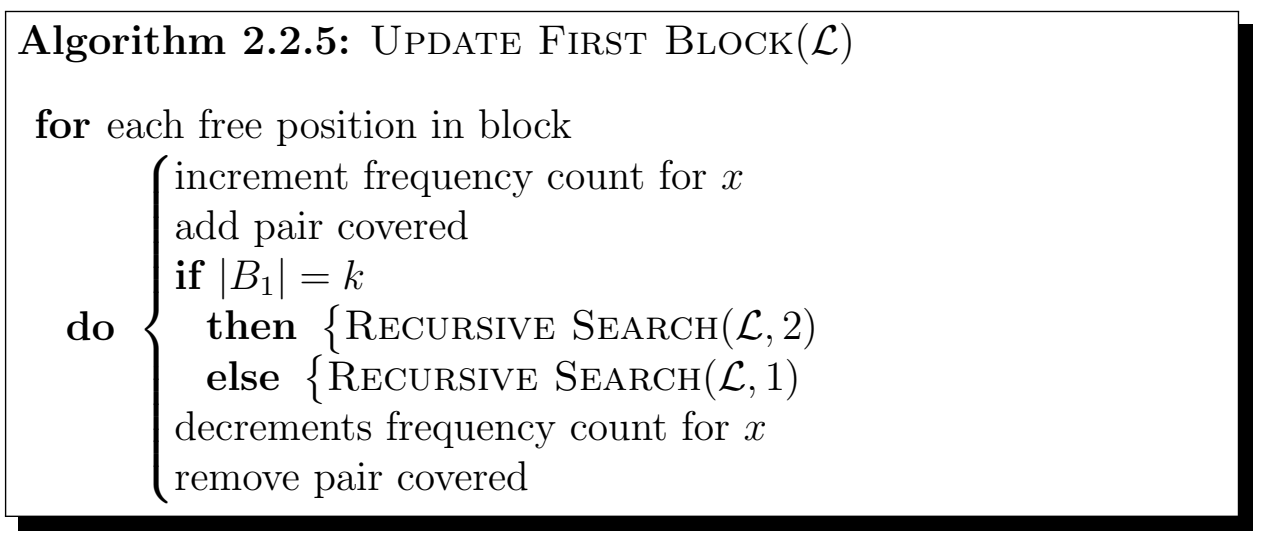

Algorithm 2.2.6: Update First Block No New $\operatorname{Points}(\mathcal{L})$ add pairs involving $x$ increment $x$ introduction and frequency count if $\left|B_{1}\right|=k$

then $\{\operatorname{Recursive} \operatorname{Search}(\mathcal{L}, 2)$

else $\{\operatorname{Recursive} \operatorname{SeARCH}(\mathcal{L}, 1)$

remove pairs involving $x$

decrements $x$ introduction and frequency count

We found 313 tight $\operatorname{CSCCD}(12,4,2,21)$ in about 150045.29 seconds or just under 42 hours using the Carleton SageMath server which has 157GB of RAM and uses an Intel Xeon E5-2667 0 @ 2.90GHz CPU. You may find the algorithm here. https://github.com/AmandaLynnC/CSCCD_ Exhaustive_Search_Masters_Thesis

Theorem 2.8. There are 313 distinct $C S C C D(12,4,2,22)$

The 313 designs are available in the same repository.

\subsubsection{SF-arrays}

With the goal of using SF-arrays to construct circular $\operatorname{SCCD}(v, k, 3, b)$ we begin an investigation into the number and restrictions of introductions in larger sets. Let $(\mathrm{X}, \mathcal{M})$ be a tight $\operatorname{CSCCD}(v, k, s, b)$. We say that a clout is 
a set $C \subset X$ where $0<c=|C|<s$. A clout is introduced in block $B$ if $C \subseteq B$ and one element in the clout was introduced in this block. We say that $T_{i}^{c} \in X$ is the set of clouts of size $c$ that are introduced in $i$ blocks. Let $t_{i}^{c}=\left|T_{i}^{c}\right|$. Note that $T_{0}^{c}$ is the set of clouts that are never introduced.

Lemma 2.9. Each clout $C \in T_{0}^{c}$ in a tight $C S C C D$ is in every block.

Proof. Let $C \subseteq S|S|=s$. Since $C$ is never introduced but $S$ must be covered, $C$ must be in every block. This follows from the circularity.

Let $f_{c}$ be the number of blocks that contain clout $C \subset X$. If $C \in T_{i}^{c}$, each time $C$ is introduced $\left(\begin{array}{l}k-c \\ s-c\end{array}\right) s$-sets containing $C$ are covered. In each remaining $f_{C}-i$ blocks that contain $C\left(\begin{array}{c}k-c-1 \\ s-c-1\end{array}\right) s$-set containing $C$ are covered.

Proposition 2.10. The number of blocks containing any clout introduced $i$ times is constant.

Proof. We know $\left(\begin{array}{l}v-c \\ s-c\end{array}\right)=i\left(\begin{array}{l}k-c \\ s-c\end{array}\right)+\left(\begin{array}{l}k-c-1 \\ s-c-1\end{array}\right)\left(f_{C}-i\right)$. In other words. $f_{C}=$ $\left(\begin{array}{l}v-c \\ s-c\end{array}\right)-c-i\left(\left(\begin{array}{l}k-c \\ s-c\end{array}\right)-\left(\begin{array}{c}k-c-s \\ s-c-1\end{array}\right)\right)$, a constant.

Let $f_{i}^{c}=\left(\begin{array}{l}v-c \\ s-c\end{array}\right)-c-i\left(\left(\begin{array}{l}k-c \\ s-c\end{array}\right)-1\right)$ be the number of blocks containing any fixed clout introduced $i$ times.

When considering clouts of size 1, we may drop the super script. For $C \in T_{1}^{c}$, let $B_{i}$ be the block where $C$ is introduced and $B_{j}$ be the last block containing $C$. We call the blocks $\left(B_{i}, \ldots, B_{j}\right)$ the run of $C$ which starts at $B_{i}$ and finishes at $B_{j}$. We will further explore properties of these clouts depending upon their size and strength of the design. When we need to clarify which clout the $S$ or $F$ of a SF-array corresponding to we will specify with $S_{C}$ and $F_{C}$.

\subsection{Higher Strength SCCD}

Moving forward, we consider sets of strength $s>2$. Let $g_{1}(v, k, s)=$ $\frac{\left(\begin{array}{l}v \\ s\end{array}\right)-\left(\begin{array}{l}k \\ s\end{array}\right)}{\left(\begin{array}{l}k-1 \\ s-1\end{array}\right)}+1$. Let $g_{2}(v, k, s)=\frac{\left(\begin{array}{l}v \\ s\end{array}\right)}{\left(\begin{array}{l}k-1 \\ s-1\end{array}\right)}$.

Proposition 2.11. The number of blocks in a $S C C D(v, k, s, b)$ is at least $b \geq\left\lceil g_{1}(v, k, s)\right\rceil$. The number of blocks in a $C S C C D(v, k, s, b)$ is at least $b \geq\left\lceil g_{2}(v, k, s)\right\rceil$. 
Proof. Suppose we have a $\operatorname{SCCD}(v, k, s, b),(X, \mathcal{L})$. The first block of $\mathcal{L}$ will cover $\left(\begin{array}{l}k \\ s\end{array}\right) s$-sets and each subsequent block will cover at most $\left(\begin{array}{l}k-1 \\ s-1\end{array}\right)$ new $s$-sets. There are $\left(\begin{array}{l}v \\ s\end{array}\right) s$-sets which must be covered, therefore

$$
\begin{aligned}
\left(\begin{array}{l}
v \\
s
\end{array}\right) & \leq\left(\begin{array}{l}
k \\
s
\end{array}\right)+(b-1)\left(\begin{array}{l}
k-1 \\
s-1
\end{array}\right) \\
b & \geq\left\lceil\frac{\left(\begin{array}{l}
v \\
s
\end{array}\right)-\left(\begin{array}{l}
k \\
s
\end{array}\right)}{\left(\begin{array}{l}
k-1 \\
s-1
\end{array}\right)}+1\right\rceil .
\end{aligned}
$$

Suppose we have a $\operatorname{CSCCD}(v, k, s, b),(X, \mathcal{M})$. Each block of $\mathcal{M}$ will cover at most $\left(\begin{array}{l}k-1 \\ s-1\end{array}\right)$ new $s$-sets. There are $\left(\begin{array}{l}v \\ s\end{array}\right) s$-sets which must be covered. Therefore,

$$
\begin{aligned}
\left(\begin{array}{l}
v \\
s
\end{array}\right) & \leq b\left(\begin{array}{l}
k-1 \\
s-1
\end{array}\right) \\
b & \geq\left\lceil\frac{\left(\begin{array}{l}
v \\
s
\end{array}\right)}{\left(\begin{array}{l}
k-1 \\
s-1
\end{array}\right)}\right\rceil .
\end{aligned}
$$

Corollary 2.12. A $C S C C D(v, k, 3, b)$ will have at least $b=\left\lceil\frac{v(v-1)(v-2)}{3(k-1)(k-2)}\right\rceil$ blocks.

We say a (circular) $\operatorname{SCCD}(v, k, s, b)$ is economical if it has $\left\lceil g_{1}(v, k, s)\right\rceil$ $\left(\left\lceil g_{2}(v, k, s)\right\rceil\right)$ blocks and tight if it is economical and $g_{1}(v, k, s)\left(g_{2}(v, k, s)\right)$ is an integer. $\operatorname{A~} \operatorname{SCCD}(v, k, s, b)$ is minimal if there does not exist a $\operatorname{SCCD}(v$, $\left.k, s, b^{\prime}\right), \forall b^{\prime}<b$. We say that a block $B_{i}$ in a $\operatorname{SCCD}(b, k, s, b)$ is tight if the pairs it covers are not covered in any other block of the SCCD.

The definition of unchanged subset of a $\operatorname{SCCD}(v, k, s)$ remains the same. Let $W \subseteq X,|W|=w$. A set $\mathcal{E}=\left\{U_{i_{1}}, \ldots, U_{i_{l}}\right\}, l=\frac{\left(\begin{array}{c}w \\ s-1\end{array}\right)}{\left(\begin{array}{c}k-1 \\ s-1\end{array}\right)}$ of unchanged subsets which form the blocks of a $S(w, k-1, s-1)$ design is a $\mathbf{W}$-expansion set. A V-expansion set is an expansion set. If $\mathcal{E}$ contains neither $u_{0}$ or $u_{b}$ then it is an inner expansion set. If $\mathcal{E}$ contains $u_{0}, u_{b}$, or both, then it is an outer expansion set. We will be denoting expansion locations with a caret, $\wedge$. This generalized definition is key in some constructions in order to preserve tightness. 
Theorem 2.13. Let $(X, \mathcal{L})$ be a $S C C D(v, k, s, b)$ with an expansion set. Then a $\left(X^{\prime}, \mathcal{L}^{\prime}\right) S C C D\left(v+1, k, s, b+\frac{\left(\begin{array}{c}v \\ s-1\end{array}\right)}{\left(\begin{array}{c}k-1 \\ s-1\end{array}\right)}\right)$ exists. Moreover, if $(X, \mathcal{L})$ is tight, economic, or circular then so is $\left(X^{\prime}, \mathcal{L}^{\prime}\right)$.

Proof. Let $(X, \mathcal{L})$ be a $\operatorname{SCCD}(v, k, s, b)$ with an expansion set $\mathcal{E}=\left\{U_{i_{1}}, \ldots\right.$, $\left.U_{i_{l}}\right\}, l=\frac{\left(\begin{array}{c}v \\ s-1\end{array}\right)}{\left(\begin{array}{c}k-1 \\ s-1\end{array}\right)}$. We build $\left(X^{\prime}, \mathcal{L}^{\prime}\right)$ by inserting a new block $B_{j}^{\prime}=U_{i_{j}} \cup\{x\}, 1 \leq$ $j \leq l$ at expansion set location $i_{j}$. The blocks we insert are single change so $\left(X^{\prime}, \mathcal{L}^{\prime}\right)$ is single change. The $s$-sets covered in $\mathcal{L}$ will be covered in $\mathcal{L}^{\prime}$ in the same blocks. The $s$-sets of the form $\{x\} \cup S,|S|=s-1, S \subseteq U_{i}, 1 \leq j \leq l$ will be covered in $B_{j}^{\prime}$. As $|S|=s-1$, there exists a unique unchanged subset that contains $S$, since an expansion set is a design of strength $s-1$. So the $s$ sets of the form $\{x\} \cup S$ are covered in $B_{j}^{\prime}$. The new blocks only cover $s$-sets containing $x$ and none are repeated, so if $(X, \mathcal{L})$ is tight then $\left(X^{\prime}, \mathcal{L}^{\prime}\right)$ is tight. The same is true for the economical case. As the unchanged subsets of the first and last blocks of $\left(X^{\prime}, \mathcal{L}^{\prime}\right)$ are the same as those in $(X, \mathcal{L})$, the new blocks do not alter circularity.

We proved the analogue of this construction for $s=2$ in my undergraduate honours thesis [3].

Conjecture 2.14. Let $\left(X, \mathcal{L}=\left(B_{1}, \ldots, B_{b}\right)\right)$ be an economical $S C C D(v, k, s, b)$. Suppose that $\left(X^{\prime}, \mathcal{L}^{\prime}=\left(B_{1}, \ldots, B_{b}, B_{b+1}^{\prime}, \ldots, B_{b^{\prime}}^{\prime}\right)\right)$ is a $S C C D\left(v^{\prime}, k, s, b^{\prime}\right) \quad(C S C C D(v, k, s, b))$ and $X \subseteq X^{\prime}$. If $B_{i}^{\prime}$ is tight for all $1 \leq i \leq b^{\prime}$, and a tight $S C C D(v, k, s, b)$ (tight $\left.C S C C D(v, k, s, b)\right)$ exists in $\left(B_{b+1}^{\prime}, \ldots, B_{b^{\prime}}^{\prime}\right)$ then $\left(X^{\prime}, \mathcal{L}^{\prime}\right)$ is economical.

For the rest of Chapter 2 we focus on $s=3$.

Theorem 2.15. Let $(X, \mathcal{L})$ be a $\operatorname{SCCD}(v, k, 3, b)$ with an outer expansion set and $\left(X^{\prime}, \mathcal{L}^{\prime}\right)$ be a $S C C D\left(v^{\prime}, k, 3, b^{\prime}\right)$ with $X^{\prime} \cap X=U_{b} \in B_{1}^{\prime}$ and $\left(X^{\prime} \backslash X\right)$ expansion set. Then there exists a $\operatorname{SCCD}\left(v^{*}, k, 3, b^{*}\right),\left(X^{*}, \mathcal{L}^{*}\right)$, with $v^{*}=$ $\left(v+v^{\prime}-(k-1)\right)$ and $b^{*}=v+v^{\prime}+\frac{v(v-1)}{(k-1)(k-2)}\left(v^{\prime}-k+1\right)+\frac{\left(v^{\prime}-k+1\right)\left(v^{\prime}-k\right)}{(k-1)(k-2)}(v-k+1)$. Moreover,

- If $(X, \mathcal{L})$ and $\left(X^{\prime}, \mathcal{L}^{\prime}\right)$ are tight then $\left(X^{*}, \mathcal{L}^{*}\right)$ is tight.

- If Conjecture 2.14 holds then if $(X, \mathcal{L})$ is tight and $\left(X^{\prime}, \mathcal{L}^{\prime}\right)$ is economical then $\left(X^{*}, \mathcal{L}^{*}\right)$ is economical. 
Proof. Let $(X, \mathcal{L})$ be a $\operatorname{SCCD}(v, k, 3, b)$ with outer expansion set $\mathcal{E}=\left\{U_{i_{j}}\right.$ : $1 \leq j \leq n\}, n=\frac{v(v-1)}{(k-1)(k-2)}$ and $U_{b} \in \mathcal{E}$. Let $\left(X^{\prime}, \mathcal{L}^{\prime}\right)$ be $\operatorname{SCCD}\left(v^{\prime}, k, 3, b^{\prime}\right)$ with $X^{\prime} \backslash X$-expansion set, $\mathcal{E}^{\prime}=\left\{U_{i_{j}}: 1 \leq j \leq n^{\prime}\right\}, n^{\prime}=\frac{\left(v^{\prime}-k+1\right)\left(v^{\prime}-k\right)}{(k-1)(k-2)}$

We construct $\left(X^{\prime} \cup X, \mathcal{L}^{*}\right)$. We start by appending $\mathcal{L}^{\prime}$ to $\mathcal{L}$. As $U_{b} \subset B_{b}$ and $U_{b} \subset B_{1}^{\prime}$, this list of blocks is also single change. Next, insert $m^{\prime}=$ $v^{\prime}-k+1$ blocks, $F_{i_{f}}$, of the form $U_{i} \cup\{f\}$, for all $f \in X^{\prime} \backslash X$ at each expansion location in $\mathcal{L}$. Finally, insert $m=v-k+1$ blocks, $G_{i_{g}}$, of the form $U_{i}^{\prime} \cup\{g\}$ for all $g \in X \backslash X^{\prime}$ at each $W$-expansion location in $\mathcal{L}^{\prime}$. With this we cover all triples:

1. The triples containing three elements of $X$ are all covered in blocks $\mathcal{L}$.

2. The triples containing only elements of $X^{\prime} \backslash X$ are covered in blocks $\mathcal{L}^{\prime}$.

3. The triples containing two points from $X \backslash X^{\prime}$ and one point from $X^{\prime} \backslash X$ are covered in blocks $F$ inserted at expansion locations in $\mathcal{L}$.

4. The triples containing two points from $X^{\prime} \backslash X$ and one point from $X \backslash X^{\prime}$ are covered in blocks $G$ inserted at expansion locations in $\mathcal{L}^{\prime}$.

5. The triples containing two points from $X \cap X^{\prime}$ and one point from $X^{\prime} \backslash X$ are covered in $\mathcal{L}^{\prime}$.

6. The triples containing two points from $X^{\prime} \backslash X$ and one point from $X \cap X$ are covered in $\mathcal{L}^{\prime}$.

7. The triples containing one point each from $X \backslash X^{\prime}, X^{\prime} \backslash X$, and $X \cap X^{\prime}$, are covered in the blocks $F$ inserted at expansion locations in $\mathcal{L}$.

As $U_{i_{j}} \subset F_{i_{f}}$ and $U_{i}^{\prime} \subset G_{i_{g}}$ for all $F$ and $G$ the unchanged subsets are not altered. If the blocks of $\mathcal{L}$ and $\mathcal{L}^{\prime}$ were tight then $\mathcal{L}^{*}$ is tight as no blocks of $F_{i_{f}}$ or $G_{i_{g}}$ re-cover any pair. Therefor if $(X, \mathcal{L})$ and $\left(X^{\prime}, \mathcal{L}^{\prime}\right)$ were tight, so is $\left(X^{*}, \mathcal{L}^{*}\right)$. 


\section{Chapter 3}

\section{Double Change Covering Designs}

We now explore designs where more than one element changes between blocks. For $m<k<v$, a multi-change covering design $(\mathrm{m}-\mathrm{CCD}(v, k, s$, $b)),(X, \mathcal{L})$, is a $v$-set $X$ and an ordered list of blocks $\mathcal{L}=\left(B_{1}, \ldots, B_{b}\right)$ of size $k$ where every $s$-set, $S$, must occur in at least one block. Every block except the last differs from the next block by removing $m$ elements and introducing $m$ elements. So $\left|B_{i} \cap B_{i+1}\right|=(k-m)$ for all $1 \leq i<b$ and the points of $B_{i+1} \backslash B_{i}$ are introduced in $B_{i+1}$ and $B_{i} \backslash B_{i+1}$ are removed in $B_{i}$. If $\left|B_{1} \cap B_{b}\right|=k-m$ then the SCCD is circular and $m$ points are introduced in each block. In a non circular m-CCD every point in $B_{1}$ is introduced in $B_{1}$. A $s$-set, $S$, is covered on block $B_{i}$ if $S \subseteq B_{i}$ and at least one element of $S$ was introduced in $B_{i}$. If no $\operatorname{DCCD}\left(v, k, s, b^{\prime}\right)$ exists for all $b^{\prime}<b$ then a $\operatorname{DCCD}(v, k, s, b)$ is called minimal.

We say that $U_{i}=B_{i} \cap B_{i+1}$ is the unchanged subset between these blocks. If the m-CCD is not circular we can take the unchanged subsets $U_{0}$ and $U_{b}$ to be any $(k-m)$ subset of $B_{1}$ and $B_{b}$ respectively. If $W \subseteq X$ and

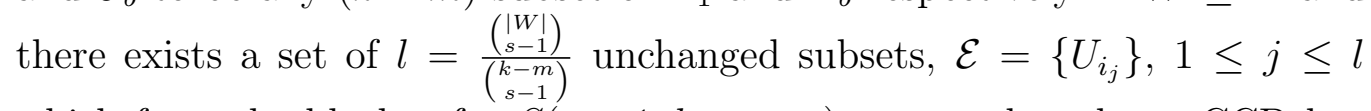
which form the blocks of a $S(s-1, k-m, v)$ we say that the m-CCD has an $\mathbf{W}$-expansion set. If neither $U_{0}, U_{b} \in \mathcal{E}$ then $\mathcal{E}$ is an inner expansion set, otherwise $\mathcal{E}$ is an outer expansion set. We denote expansion locations with a caret, $\wedge$.

Similarly to single change covering designs we define lower bounds on the 
number of blocks. Let $g_{1}^{\prime}(v, k, s, m)=\frac{\left(\begin{array}{c}v \\ s\end{array}\right)-\left(\begin{array}{c}k \\ s\end{array}\right)}{\sum_{i=0}^{s}\left(\begin{array}{c}m \\ i\end{array}\right)\left(\begin{array}{c}k-m \\ s-i\end{array}\right)}+1$, and $g_{2}^{\prime}(v, k, s, m)=$ $\frac{\left(\begin{array}{c}v \\ s\end{array}\right)}{\sum_{i=0}^{s}\left(\begin{array}{c}m \\ i\end{array}\right)\left(\begin{array}{c}k-m \\ s-i\end{array}\right)}$.

Theorem 3.1. A m-CCD $(v, k, s, b)$ will have $b \geq\left\lceil g_{1}^{\prime}(v, k, s, m)\right\rceil$ blocks. A circular $m-C C D$ will have at least $b=\left\lceil g_{2}^{\prime}(v, k, s, m)\right\rceil$ blocks.

Proof. The first block of the m-CCD will cover $\left(\begin{array}{l}k \\ s\end{array}\right) s$-sets. Every other block will have $m$ elements introduced. The number of $s$-sets that contain $i$ of the $m$ introduced elements and $s-i$ of the remaining $k-m$ points is $\left(\begin{array}{c}m \\ i\end{array}\right)\left(\begin{array}{c}k-m \\ s-i\end{array}\right)$. Hence $\sum_{i=0}^{s}\left(\begin{array}{c}m \\ i\end{array}\right)\left(\begin{array}{c}k-m \\ s-i\end{array}\right) s$-sets are introduced in each block after the first. There are $\left(\begin{array}{l}v \\ s\end{array}\right) s$-sets to cover so $\left(\begin{array}{l}v \\ s\end{array}\right) \leq\left(\begin{array}{l}k \\ s\end{array}\right)+(b-1)\left(\sum_{i=0}^{s}\left(\begin{array}{c}m \\ i\end{array}\right)\left(\begin{array}{c}k-m \\ s-i\end{array}\right)\right)$

In the circular case, each block covers at most $\sum_{i=0}^{s}\left(\begin{array}{c}m \\ i\end{array}\right)\left(\begin{array}{c}k-m \\ s-i\end{array}\right)$ new $s$-sets and the calculation is similar.

We say a (circular) $\mathrm{m}-\mathrm{CCD}(v, k, s, b)$ is economical if it has $\left\lceil g_{1}^{\prime}(v, k, s, m)\right\rceil\left(\left\lceil g_{2}^{\prime}(v, k, s, m)\right\rceil\right)$ blocks and tight if it is economical and $g_{1}^{\prime}(v, k, s, m)\left(g_{2}^{\prime}(v, k, s, m)\right)$ is an integer. We say that a block $B_{i}$ in a DCCD is tight if the $s$-sets the block covers are not covered in any other block of the DCCD.

We will be focusing on designs with $m=2$ and will call these double change covering designs $(\operatorname{DCCD}(v, k, s, b))$. When $m=2$ we get $g_{1}^{\prime}(v, k, s, 2)=\frac{\left(\begin{array}{l}v \\ s\end{array}\right)-\left(\begin{array}{l}k \\ s\end{array}\right)}{2\left(\begin{array}{l}k-2 \\ s-1\end{array}\right)+\left(\begin{array}{l}k-2 \\ s-2\end{array}\right)}+1$ and $g_{2}^{\prime}(v, k, s, 2)=\frac{\left(\begin{array}{l}v \\ s\end{array}\right)}{2\left(\begin{array}{c}k-2 \\ s-1\end{array}\right)+\left(\begin{array}{c}k-2 \\ s-2\end{array}\right)}$. When $m=$ $s=2$ we get $g_{1}^{\prime}(v, k, 2,2)=\frac{v(v-1)-k(k-1)}{4 k-6}+1$ and $g_{2}^{\prime}(v, k, 2,2)=\frac{v(v-1)}{4 k-6}$.

An example of a $\operatorname{CDCCD}(7,3,2,7)$ is given in Table 3.1. The blocks are those of $S T S(7)$. It is tight as either a DCCD or CDCCD. The unchanged subsets for the non-circular (circular) DCCD are $U_{1}=\{0\}, U_{2}=\{4\}, U_{3}=$ $\{3\}, U_{4}=\{6\}, U_{5}=\{6\}, U_{6}=\{5\}$ where $U_{0}=\{\{0\},\{1\},\{2\}\}$ and $U_{7}=$ $\{\{1\},\{3\},\{5\}\}\left(U_{1}=\{0\}, U_{2}=\{4\}, U_{3}=\{3\}, U_{4}=\{6\}, U_{5}=\{6\}, U_{6}=\right.$ $\left.\{5\}, U_{0}=U_{7}=\{1\}\right)$. Only the non-circular $\operatorname{DCCD}(7,3,2,7)$ has an expansion set. 


$\begin{array}{cllllll}\mathrm{B}_{1} & \mathrm{~B}_{2} & \mathrm{~B}_{3} & \mathrm{~B}_{4} & \mathrm{~B}_{5} & \mathrm{~B}_{6} & \mathrm{~B}_{7} \\ 0^{*} & 0 & 2^{*} & 0^{*} & 1^{*} & 5^{*} & 5 \\ 1 & 4^{*} & 4 & 6^{*} & 6 & 6 & 3^{*} \\ 2^{*} \wedge & 5^{*} \wedge & 3^{*} \wedge & 3 & 4^{*} \wedge & 2^{*} \wedge & 1^{*} \wedge\end{array}$

Table 3.1: A tight $\operatorname{CDCCD}(7,3,2,7)$

Theorem 3.2. There exists a tight $D C C D(v, 3,2, b)$ and tight $C D C C D(v, 3,2, b)$ for all $v \equiv 1,3(\bmod 6)$.

Proof. There exists a $\operatorname{STS}(v)$ for all $v=1,3(\bmod 6)[13]$. Horak and Rosa proved the 1 block intersection graph of a $\operatorname{STS}(v)$ is Hamiltonian [7].

Let $C=\left\{B_{1}, B_{2}, \ldots, B_{b}\right\}$ be the Hamilton cycle in the 1-block intersection graph of a $\operatorname{STS}(v)$. As $\left|B_{i} \cap B_{i+1}\right|=1$, the double change condition is met on $C$. As the blocks are those of a $\operatorname{STS}(v)$, every pair is covered and the number of blocks is $b=\frac{v(v-1)}{6}$ which matches both $g_{1}^{\prime}$ and $g_{2}^{\prime}$

Conjecture 3.3. Let $\left(X, \mathcal{L}=\left(B_{1}, \ldots, B_{b}\right)\right)$ be an economic $D C C D(v, k, 2, b)$ with $d=b\left(2\left(\begin{array}{c}k-2 \\ s-1\end{array}\right)+\left(\begin{array}{c}k-2 \\ s-2\end{array}\right)\right)+\left(\begin{array}{l}k \\ s\end{array}\right)-\left(\begin{array}{l}v \\ s\end{array}\right)$ blocks. Suppose that $\left(X^{\prime}, \mathcal{L}^{\prime}=\right.$ $\left.\left(B_{1}, \ldots, B_{b}, B_{b+1}^{\prime}, \ldots, B_{b^{\prime}}^{\prime}\right)\right)$ is a $S C C D\left(v^{\prime}, k, 2, b^{\prime}\right)$ with $X \subseteq X^{\prime}$. If $B_{i}^{\prime}$ is tight $\forall i, b+1 \leq i \leq b^{\prime}$, then $\left(X^{\prime}, \mathcal{L}^{\prime}\right)$ has $b^{\prime}\left(2\left(\begin{array}{c}k-2 \\ s-1\end{array}\right)+\left(\begin{array}{c}k-2 \\ s-2\end{array}\right)\right)+\left(\begin{array}{l}k \\ s\end{array}\right)-\left(\begin{array}{l}v^{\prime} \\ s\end{array}\right)=d$ blocks.

Theorem 3.4. Let $(X, \mathcal{L})$ be a $D C C D(v, k, 2, b)$ with $v$ an odd multiple of $k-2$ and expansion set $\mathcal{E}$. Then a $D C C D\left(v+\frac{v}{k-2}+1, k, 2, b+\frac{v}{k-2} \frac{v}{2}\right),\left(X^{*}, \mathcal{L}^{*}\right)$, exists. Moreover, if $(E, \mathcal{L})$ is tight or circular then $\left(X^{*}, \mathcal{L}^{*}\right)$ is too. If Conjecture 3.3 holds then if $(X, \mathcal{L})$ is economical then $\left(X^{*}, \mathcal{L}^{*}\right)$ is economic.

Proof. Let $(X, \mathcal{L})$ be a $\operatorname{DCCD}(v, k, 2, b)$ with the expansion set $\mathcal{E}=\left\{U_{i_{j}}\right.$ : $\left.1 \leq j \leq t \leq \frac{v}{k-2}\right\}$. Let $\left\{F_{j}\right\}_{1}^{t}$ be a 1-factorization of $K_{t+1}$ on set $V[1]$.

For each edge $e \in F_{j}$ let $B_{j, e}=U_{i_{j}} \cup\{e\}$. Insert $B_{j, e}$ at each expansion location $U_{i_{j}} \in \mathcal{E}$. These insertions maintain the double change property. All the pairs that were covered in $(X, \mathcal{L})$ are still covered in $\mathcal{L}$. Since each pair of elements from $V$ is in exactly one 1-factor all the pairs in $V$ are covered. The blocks inserted at expansion set locations ensure each pair of elements $\{x, y\}, x \in X, y \in V$ is covered. So $\left(X^{*}, \mathcal{L}^{*}\right)$ is a $\operatorname{DCCD}\left(v+\frac{v}{k-2}+1, k, 2, b^{\prime}\right)$ with $b^{\prime}=b+\frac{v^{2}}{2 k-4}$. 
Each $B_{j_{e}}$ is tight, so if $(X, \mathcal{L})$ was tight so is $\left(X^{*}, \mathcal{L}^{*}\right)$. The block insertions do not affect the double change between the first and last block, so if $(X, \mathcal{L})$ was circular so is $\left(X^{*}, \mathcal{L}^{*}\right)$.

We note that every new element $x$ added to $V$ in Theorem 3.4 is introduced in $B_{i}$ and removed in $B_{i+1}$ in $\mathcal{L}^{*}$. Consequently, $x$ is never in an unchanged subset and any $\mathcal{L}^{*}$ constructed in this manner will never have an expansion set. Thus, we looked for recursions that will preserve expansion sets similar to when we use two SCCD to construct a larger SCCD as in Theorem 1.38.

Theorem 3.5. Let $(X, \mathcal{L})$ and $(\tilde{X}, \tilde{\mathcal{L}})$ be a $\operatorname{DCCD}(v, k, 2, b)$. Let $\left(X^{\prime}, \mathcal{L}^{\prime}\right)$ be a $C D C C D\left(v^{\prime}, k, 2, b^{\prime}\right)$ with an outer expansion set where $v^{\prime}=\frac{v-k+2}{k-2}+2 k-4$. Then a $D C C D\left(v^{*}, k, 2, b^{*}\right), b^{*}=2 b+b^{\prime}+(v-k+2)^{2},\left(X^{*}, \mathcal{L}^{*}\right)$, exists. Furthermore,

- If $(X, \mathcal{L})$ and $(\tilde{X}, \tilde{\mathcal{L}})$ are circular then $\left(X^{*}, \mathcal{L}^{*}\right)$ is circular.

- If $(X, \mathcal{L}),(\tilde{X}, \tilde{\mathcal{L}})$, and $\left(X^{\prime}, \mathcal{L}^{\prime}\right)$ are tight $($ circular $)$ then $\left(X^{*}, \mathcal{L}^{*}\right)$ will only re-cover at most $k-2($ at most $3 k-6)$ pairs.

Proof. Let $(X, \mathcal{L})$ and $(\tilde{X}, \tilde{\mathcal{L}})$ be two $\operatorname{DCCD}(v, k, 2, b)$. Let $n=v-k+2$ and $\left(X^{\prime}, \mathcal{L}^{\prime}\right)$ be a $\operatorname{CDCCD}\left(v^{\prime}, k, 2, b^{\prime}\right)$ with an outer expansion set $\mathcal{E}=\left\{U_{i_{j}}\right.$ : $1 \leq j \leq n\}$, and $v^{\prime}=\frac{v-k+2}{k-2}+2 k-4$ and $X^{\prime} \cap X=U_{0}^{\prime}=U_{b}^{\prime}=X^{\prime} \cap \tilde{X}$. Take $\mathcal{L}^{*}=\mathcal{L} \cup \mathcal{L}^{\prime} \cup \tilde{\mathcal{L}}$ such that $\left|X \backslash X^{\prime}\right|=|\tilde{X} \backslash X|=n$. Let $\left\{F_{j}\right\}_{1}^{t}$ be a 1-factorization of $K_{n, \tilde{n}}$ on set $\left\{X \backslash X^{\prime}, \tilde{X} \backslash X^{\prime}\right\}$ [1].

For each edge $e \in F_{j}$ let $B_{j_{e}}=U_{i_{j}} \cup e$. Insert $B_{j_{e}}$ at each expansion location $U_{i_{j}} \in \mathcal{E}$.

These insertions maintain the double change property. All the pairs that were covered in $(X, \mathcal{L})$ are covered in $\mathcal{L}$. All the pairs that were covered in $\left(X^{\prime}, \mathcal{L}^{\prime}\right)$ are covered in $\mathcal{L}^{\prime}$. All the pairs that were covered in $(\tilde{X}, \tilde{\mathcal{L}})$ are covered in $\tilde{\mathcal{L}}$. The pairs $\{x, \tilde{x}\},\left\{x^{\prime}, \tilde{x}\right\},\left\{x, x^{\prime}\right\}$ where $x \in X \backslash X^{\prime}, x^{\prime} \in$ $X^{\prime} \backslash(X \cup \tilde{X})$, and $\tilde{x} \in \tilde{X} \backslash X^{\prime}$ are covered in $B_{j_{e}}^{\prime}$.

As The pairs $\{x, y\}$ where $x, y \in X \cap X^{\prime} \cap \tilde{X}$ are covered in both $\mathcal{L}$ and $\mathcal{L}^{\prime}$. These are the only pairs of elements that may repeat their coverings. So for $\mathcal{L}, \tilde{\mathcal{L}}$ non-circular there are at most $v-k+2$ pairs that are re-covered. For $\mathcal{L}, \tilde{\mathcal{L}}$ circular we now have $\tilde{U}_{b}=U_{0}=U_{b}=U_{0}^{\prime}=U_{b}^{\prime}=\tilde{U}_{0}$, so the pairs $\{x, y\}$ where $x, y \in X \cap X^{\prime} \cap \tilde{X}$ are covered in $\mathcal{L}, \mathcal{L}^{\prime}$ and $\tilde{\mathcal{L}}$ and are recovered at most $v-3 k+6$ times. 
For example consider the non-circular $\operatorname{DCCD}(7,3,2,7)$ and expansion set in Table 3.1. We may insert four new blocks at the expansion set locations that consist of the unchanged subset and the vertices of an edge in a 1-factor of $K_{8}$ in Figure 3.1 to construct at $\operatorname{DCCD}(13,3,2,35)$ as seen in Table 3.2.

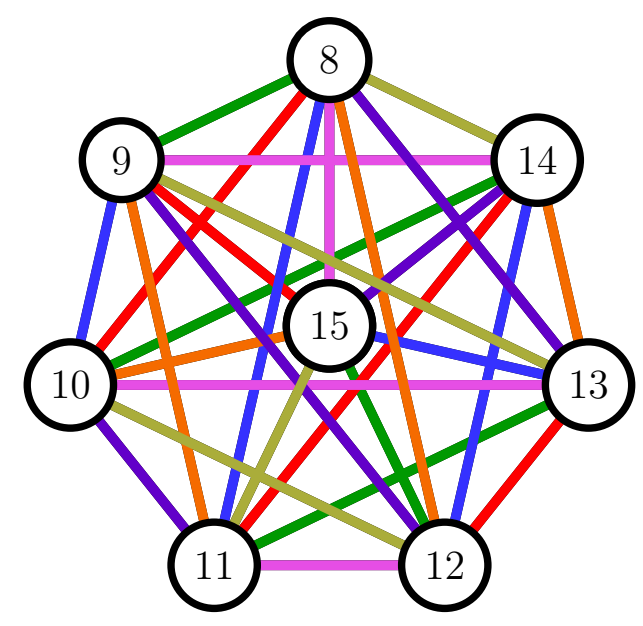

Figure 3.1: $\mathrm{K}_{8}$

\begin{tabular}{llllllllllllllllll}
$\mathrm{B}_{1}$ & $\mathrm{~B}_{2}$ & $\mathrm{~B}_{3}$ & $\mathrm{~B}_{4}$ & $\mathrm{~B}_{5}$ & $\mathrm{~B}_{6}$ & $\mathrm{~B}_{7}$ & $\mathrm{~B}_{8}$ & $\mathrm{~B}_{9}$ & $\mathrm{~B}_{10}$ & $\mathrm{~B}_{11}$ & $\mathrm{~B}_{12}$ & $\mathrm{~B}_{13}$ & $\mathrm{~B}_{14}$ & $\mathrm{~B}_{15}$ & $\mathrm{~B}_{16}$ & $\mathrm{~B}_{17}$ & $\mathrm{~B}_{18}$ \\
8 & 10 & 11 & 12 & $0^{*}$ & 0 & 0 & 0 & 0 & 0 & 8 & 9 & 12 & 13 & $2^{*}$ & 8 & 9 & 10 \\
9 & 14 & 13 & 15 & $1^{*}$ & 8 & 11 & 12 & 9 & $4^{*}$ & 4 & 4 & 4 & 4 & 4 & 15 & 14 & 13 \\
2 & 2 & 2 & 2 & 2 & 10 & 14 & 13 & 15 & $5^{*}$ & 11 & 10 & 14 & 15 & $3^{*}$ & 3 & 3 & 3 \\
\hline $\mathrm{B}_{19}$ & $\mathrm{~B}_{20}$ & $\mathrm{~B}_{21}$ & $\mathrm{~B}_{22}$ & $\mathrm{~B}_{23}$ & $\mathrm{~B}_{24}$ & $\mathrm{~B}_{25}$ & $\mathrm{~B}_{26}$ & $\mathrm{~B}_{27}$ & $\mathrm{~B}_{28}$ & $\mathrm{~B}_{29}$ & $\mathrm{~B}_{30}$ & $\mathrm{~B}_{31}$ & $\mathrm{~B}_{32}$ & $\mathrm{~B}_{33}$ & $\mathrm{~B}_{34}$ & $\mathrm{~B}_{35}$ \\
11 & $0^{*}$ & $1^{*}$ & 8 & 13 & 9 & 10 & $5^{*}$ & 5 & 5 & 5 & 5 & 5 & 8 & 9 & 10 & 11 \\
12 & $6^{*}$ & 6 & 6 & 6 & 6 & 6 & 6 & 8 & 9 & 10 & 14 & $3^{*}$ & 14 & 13 & 12 & 15 \\
3 & 3 & $4^{*}$ & 12 & 14 & 11 & 15 & $2^{*}$ & 13 & 12 & 11 & 15 & $1^{*}$ & 1 & 1 & 1 & 1
\end{tabular}

Table 3.2: A DCCD $(15,3,2,35)$ built from the $\operatorname{DCCD}(7,3,2,2)$ and $K_{8}$

\subsection{Difference Methodology}

We can construct some $\operatorname{CDCCD}(v, k, 2, b)$ using a construction similar to difference sets and families. McSorley proved that $\{0,1, \ldots, k-1\}$ is a base block of a $\operatorname{CSCCD}(2 k-1, k, 2,2 k-1)$ over $\mathbb{Z}_{2 k-1}$ in [8]. 
Every element in the tight $\operatorname{CSCCD}(2 k-1, k, 2,2 k-1)$ is introduced exactly once. If we replace $x \in \mathbb{Z}_{2 k-1}$ in $B_{i}$ with $2 x$ and $2 x+1$ in $\mathbb{Z}_{4 k-2}$ to produce a list of $2 k-1$ blocks $B_{i}^{\prime}$ of size $2 k$ from $\mathbb{Z}_{2 k} \times\{0,1\}$ each element is still introduced once. The pairs $\{2 x, 2 x+1\}$ are covered when introduced and $\{y, z\} \in \mathbb{Z}_{4 k-2}$ are covered in block where $\left\{\left\lfloor\frac{y}{2}\right\rfloor,\left\lfloor\frac{z}{2}\right\rfloor\right\}$ where covered in $\operatorname{CSCCD}(2 k-1, k, 2,2 k-1)$. Thus

Theorem 3.6. A tight $C D C C D(4 k-2,2 k, 2,2 k-1)$ exists for all $k \geq 2$.

Proof. The only thing to check is the number of blocks and $b^{\prime}=b=2 k+1=$ $g_{2}^{\prime}(4 k-2,2 k, 2,2)$.

For example, consider the tight $\operatorname{CSCCD}(3,2,2,3)$ and construct a tight CD$\operatorname{CCD}(6,4,2,3)$ in Table 3.3 .

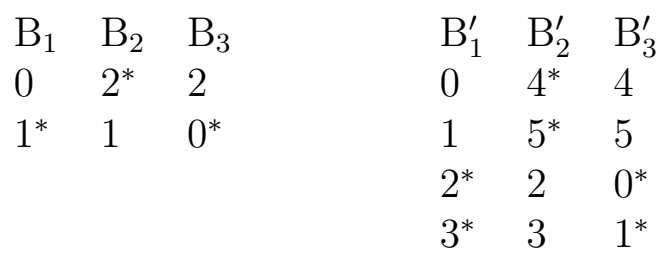

Table 3.3: A tight $\operatorname{CSCCD}(3,2,2,3)$ and tight $\operatorname{CSCCD}(6,4,2,3)$

It is worth noting that $k-1$ does not divide $2 k-1$ for $k>2$ but when $k=2$ the $\operatorname{CSCCD}(3,2,2,3)$ built this way has an expansion set, so the blown up $\operatorname{CDCCD}(6,4,2,3)$ has one as well. Using this in conjunction with the 1-factor of $K_{4}$ and Theorem 3.4 we build the $\operatorname{CDCCD}(10,4,2,9)$ seen in Table 3.4.

$\begin{array}{lllllllll}0 & \mathrm{a}^{*} & \mathrm{c}^{*} & 4^{*} & 4 & 4 & 4 & \mathrm{a}^{*} & \mathrm{~b}^{*} \\ 1 & \mathrm{~b}^{*} & \mathrm{~d}^{*} & 5^{*} & 5 & 5 & 5 & \mathrm{~d}^{*} & \mathrm{c}^{*} \\ 2^{*} & 2 & 2 & 2 & \mathrm{a}^{*} & \mathrm{~b}^{*} & 0^{*} & 0 & 0 \\ 3^{*} & 3 & 3 & 3 & \mathrm{c}^{*} & \mathrm{~d}^{*} & 1^{*} & 1 & 1\end{array}$

Table 3.4: A tight $\operatorname{CDCCD}(10,4,2,9)$

Adding a single new point to every block yields

Theorem 3.7. A tight $C D C C D(4 k-1,2 k+1,2,2 k-1)$ exists for all $k \geq 2$.

Theorem 3.8. A circular $D C C D(4 k-5, k, 2,4 k-5)$ exist for all $k$. 
Proof. Let $B_{0}=\{0,1, \ldots, k-2,2 k-3\} \subseteq \mathbb{Z}_{4 k-5}$. We construct $B_{i}=B_{0}+i \forall i \in$ $\mathbb{Z}_{4 k-5}$. Let $\mathcal{L}=\left(B_{i}\right)_{0}^{4 k-6}$. Note that $B_{j} \cap B_{j+1}=\{i+1, \ldots, k+i-2\}$ so $B_{i+1}$ covers pairs $\{k+i-1, z\}$ and $\{2 k-2+i, z\}$ for $z \in B_{i} \cap B_{i+1}$ and $\{k+i-1,2 k+i-2\}$. These pairs have differences $\pm\{1,2, \ldots, k-2, k-$ $1, k, \ldots, 2 k-3\}=\mathbb{Z}_{4 k-5} \backslash\{0\}$. As $i$ transfers $\mathbb{Z}_{4 k-5}$ every pair is covered.

$$
b=4 k-5=\frac{(4 k-5)(4 k-6)}{4 k-6}=g_{2}^{\prime}(4 k-5, k, 2,2)
$$

We can think of these vertices in a circular manor. For example, consider a $\operatorname{CDCCD}(11,4,2,11)$, so $k=4$, in Figure 3.2. The first block is $\{0,1,2,5\}$ and the points 2 and 5 are introduced. In the figure the vertices only in the first block are highlighted in blue, the vertices only on the second block are highlighted in pink, and the vertices in the unchanged subset of $B_{1}$ is in black. Now we place a line between each introduced element and the other elements in the block, blue for the first block and pink for the second. Each subsequent block is obtained by rotating the previous block by one. As this is a circle we will eventually return to our starting position and when we do we will have placed an edge between every vertex exactly once. We also see pictorially that there will only ever be double change between consecutive blocks. Note that the blue blocks cover the difference $\{1,2,3,4,5\}$ and so every block will as well.

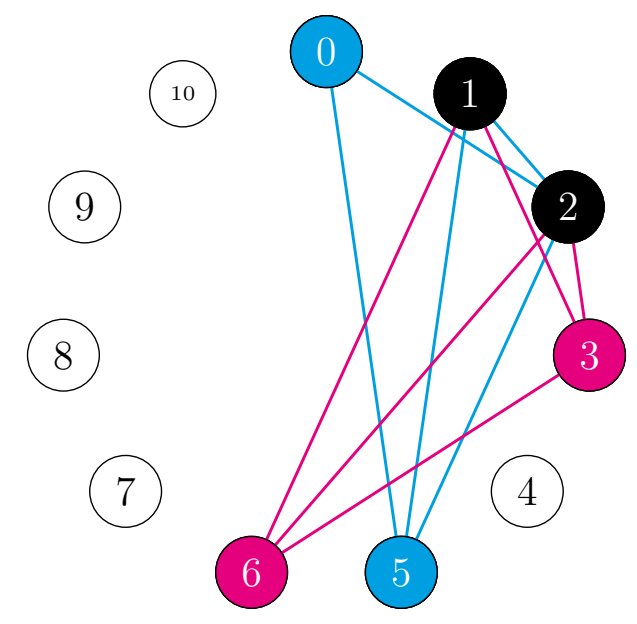

Figure 3.2: $c=1, k=4$ difference construction for $\operatorname{DCCD}(11,4,2,11)$ 
Table 3.5 shows the table form the $\operatorname{CDCCD}(11,4,2,11)$.

$\begin{array}{lllllllllll}\mathrm{B}_{1} & \mathrm{~B}_{2} & \mathrm{~B}_{3} & \mathrm{~B}_{4} & \mathrm{~B}_{5} & \mathrm{~B}_{6} & \mathrm{~B}_{7} & \mathrm{~B}_{8} & \mathrm{~B}_{9} & \mathrm{~B}_{10} & \mathrm{~B}_{11} \\ 0 & 3^{*} & 3 & 3 & 6^{*} & 6 & 6 & 9^{*} & 9 & 9 & 1^{*} \\ 1 & 1 & 4^{*} & 4 & 4 & 7^{*} & 7 & 7 & 10^{*} & 10 & 10 \\ 2^{*} & 2 & 2 & 5^{*} & 5 & 5 & 8^{*} & 8 & 8 & 0^{*} & 0 \\ 5^{*} & 6^{*} & 7^{*} & 8^{*} & 9^{*} & 10^{*} & 0^{*} & 1^{*} & 2^{*} & 3^{*} & 4^{*}\end{array}$

Table 3.5: A $\operatorname{CDCCD}(11,4,2,11)$

When $k-2 \mid 4 k-5$ these have expansion sets $\mathcal{E}=\bigcup_{i=0}^{\frac{4 k-5}{k-2}} U_{i(k-2)}$

Corollary 3.9. A tight $C D C C D(15,3,2,35)$ exists. A tight $C D C C D(21,5$, 2,30) exists.

A backtracking search yielded sets of base blocks for some parameters.

Theorem 3.10. The following tight CDCCD exist.

1. A tight $C D C C D\left(10 c+1,4,2,10 c^{2}+c\right)$ exists for all $2 \leq c \leq 6$.

2. A tight $C D C C D\left(c(4 k-6)+1, k, 2, c^{2}(4 k-6)+c\right)$ exists for all $2 \leq c \leq 4$, $5 \leq k \leq 15$.

3. A tight $C D C C D\left(c(4 k-6)+1, k, 2, c^{2}(4 k-6)+c\right)$ exists for all $2 \leq c \leq 3$, $16 \leq k \leq 20$.

Proof. To build each of the tight $\operatorname{CDCCD}\left(c(4 k-6)+1, k, 2, c^{2}(4 k-6)+c\right)$ we start the with the initial $c$ blocks given in order. Each contains $\{0,1, \ldots, k-3\}$ and $B_{0, c}$ contains $\{0,1, \ldots, k-2\}$ thus $\left(B_{0,1}, B_{0,2}, \ldots, B_{0, c}, B_{1,1}, \ldots, B_{1, c}, \ldots, B_{v, c}\right)$ is double change.

$B_{0, i}=\{0,1, \ldots, k-3, x, y\}$ covers differences $\pm\{y-x, x-k+3, \ldots, x, y-$ $k+3, \ldots, y\}$ and in each case $x, y$ can be changed such that these cover all difference in $\mathbb{Z}_{v}$

Table 3.6: Initial Blocks for some $C D C C D(c(4 k-6)+$ $\left.1, k, 2, c^{2}(4 k-6)+c\right)$

\begin{tabular}{|l|l|l|}
\hline \hline$k$ & $c$ & Initial Blocks \\
\hline \hline 4 & 2 & $\{0,1,4,12\},\{0,1,2,7\}$ \\
\hline
\end{tabular}


Table 3.6: (Initial Blocks continued)

\begin{tabular}{|l|l|l|}
\hline \hline$k$ & $c$ & Initial Blocks \\
\hline \hline 4 & 3 & $\{0,1,4,24\},\{0,1,6,22\},\{0,1,2,14\}$ \\
\hline 4 & 4 & $\{0,1,4,25\},\{0,1,6,32\},\{0,1,8,19\},\{0,1,2,14\}$ \\
\hline 4 & 5 & $\{0,1,4,17\},\{0,1,6,37\},\{0,1,8,33\},\{0,1,10,40\},\{0,1,2,24\}$ \\
\hline 4 & 6 & $\begin{array}{l}\{0,1,4,19\},\{0,1,6,22\},\{0,1,8,25\},\{0,1,10,48\},\{0,1,12,32\}, \\
\{0,1,2,28\}\end{array}$ \\
\hline 5 & 2 & $\{0,1,2,6,17\},\{0,1,2,3,10\}$ \\
\hline 5 & 3 & $\{0,1,2,6,33\},\{0,1,2,9,30\},\{0,1,2,3,20\}$ \\
\hline 5 & 4 & $\{0,1,2,6,26\},\{0,1,2,9,36\},\{0,1,2,12,40\},\{0,1,2,3,16\}$ \\
\hline 6 & 2 & $\{0,1,2,3,8,22\},\{0,1,2,3,4,13\}$ \\
\hline 6 & 3 & $\{0,1,2,3,8,17\},\{0,1,2,3,13,31\},\{0,1,2,3,4,23\}$ \\
\hline 6 & 4 & $\{0,1,2,3,8,25\},\{0,1,2,3,12,55\},\{0,1,2,3,16,47\}$, \\
& & $\{0,1,2,3,4,36\}$ \\
\hline 7 & 2 & $\{0,1,2,3,4,10,27\},\{0,1,2,3,4,5,16\}$ \\
\hline 7 & 3 & $\{0,1,2,3,4,10,21\},\{0,1,2,3,4,16,38\}$, \\
& & $\{0,1,2,3,4,5,28\}$ \\
\hline 7 & 4 & $\{0,1,2,3,4,10,26\},\{0,1,2,3,4,15,49\}$, \\
& & $\{0,1,2,3,4,21,54\},\{0,1,2,3,4,5,32\}$ \\
\hline 8 & 2 & $\{0,1,2,3,4,5,12,32\},\{0,1,2,3,4,5,6,19\}$ \\
\hline 8 & 3 & $\{0,1,2,3,4,5,12,25\},\{0,1,2,3,4,5,19,45\}$, \\
& & $\{0,1,2,3,4,5,6,33\}$ \\
\hline 8 & 4 & $\{0,1,2,3,4,5,12,25\},\{0,1,2,3,4,5,19,65\}$, \\
& & $\{0,1,2,3,4,5,32,58\},\{0,1,2,3,4,5,6,39\}$ \\
\hline 9 & 2 & $\{0,1,2,3,4,5,6,14,37\},\{0,1,2,3,4,5,6,7,22\}$ \\
\hline 9 & 3 & $\{0,1,2,3,4,5,6,14,29\},\{0,1,2,3,4,5,6,22,52\}$, \\
& & $\{0,1,2,3,4,5,6,7,38\}$ \\
\hline 9 & 4 & $\{0,1,2,3,4,5,6,14,36\},\{0,1,2,3,4,5,6,21,67\}$, \\
& & $\{0,1,2,3,4,5,6,29,74\},\{0,1,2,3,4,5,6,7,44\}$ \\
\hline 10 & 2 & $\{0,1,2,3,4,5,6,7,16,42\},\{0,1,2,3,4,5,6,7,8,25\}$ \\
\hline 10 & 3 & $\{0,1,2,3,4,5,6,7,16,33\},\{0,1,2,3,4,5,6,7,25,59\}$, \\
& & $\{0,1,2,3,4,5,6,7,8,43\}$ \\
\hline 10 & 4 & $\{0,1,2,3,4,5,6,7,16,50\},\{0,1,2,3,4,5,6,7,24,102\}$, \\
& & $\{0,1,2,3,4,5,6,7,33,58\},\{0,1,2,3,4,5,6,7,8,68\}$ \\
\hline 11 & 2 & $\{0,1,2,3,4,5,6,7,8,18,47\},\{0,1,2,3,4,5,6,7,8,9,28\}$ \\
\hline & & \\
\hline
\end{tabular}


Table 3.6: (Initial Blocks continued)

\begin{tabular}{|l|l|l|}
\hline \hline$k$ & $c$ & Initial Blocks \\
\hline \hline 11 & 3 & $\{0,1,2,3,4,5,6,7,8,18,37\},\{0,1,2,3,4,5,6,7,8,28,66\}$, \\
& $\{0,1,2,3,4,5,6,7,8,9,48\}$ \\
\hline 11 & 4 & $\{0,1,2,3,4,5,6,7,8,18,46\},\{0,1,2,3,4,5,6,7,8,27,85\}$, \\
& $\{0,1,2,3,4,5,6,7,8,37,94\},\{0,1,2,3,4,5,6,7,8,9,56\}$ \\
\hline 12 & 2 & $\{0,1,2,3,4,5,6,7,8,9,20,52\},\{0,1,2,3,4,5,6,7,8,9,10,31\}$ \\
\hline 12 & 3 & $\{0,1,2,3,4,5,6,7,8,9,20,41\},\{0,1,2,3,4,5,6,7,8,9,31,73\}$, \\
& & $\{0,1,2,3,4,5,6,7,8,9,10,53\}$ \\
\hline 12 & 4 & $\{0,1,2,3,4,5,6,7,8,9,20,51\},\{0,1,2,3,4,5,6,7,8,9,30,94\}$, \\
& & $\{0,1,2,3,4,5,6,7,8,9,41,104\},\{0,1,2,3,4,5,6,7,8,9,10,62\}$ \\
\hline 13 & 2 & $\{0,1,2,3,4,5,6,7,8,9,10,22,57\}$, \\
& $\{0,1,2,3,4,5,6,7,8,9,10,11,34\}$ \\
\hline 13 & 3 & $\{0,1,2,3,4,5,6,7,8,9,10,22,103\}$, \\
& $\{0,1,2,3,4,5,6,7,8,9,10,57,80\}$, \\
& $\{0,1,2,3,4,5,6,7,8,9,10,11,35\}$ \\
\hline 13 & 4 & $\{0,1,2,3,4,5,6,7,8,9,10,22,45\}$, \\
& $\{0,1,2,3,4,5,6,7,8,9,10,34,115\}$, \\
& $\{0,1,2,3,4,5,6,7,8,9,10,57,103\}$, \\
& $\{0,1,2,3,4,5,6,7,8,9,10,11,69\}$ \\
\hline 14 & 2 & $\{0,1,2,3,4,5,6,7,8,9,10,11,24,62\}$, \\
& $\{0,1,2,3,4,5,6,7,8,9,10,11,12,37\}$ \\
\hline 14 & 3 & $\{0,1,2,3,4,5,6,7,8,9,10,11,24,87\}$, \\
& $\{0,1,2,3,4,5,6,7,8,9,10,11,37,62\}$, \\
& $\{0,1,2,3,4,5,6,7,8,9,10,11,12,50\}$ \\
\hline 14 & 4 & $\{0,1,2,3,4,5,6,7,8,9,10,11,24,49\}$, \\
& $\{0,1,2,3,4,5,6,7,8,9,10,11,37,125\}$, \\
& $\{0,1,2,3,4,5,6,7,8,9,10,11,62,112\}$, \\
& $\{0,1,2,3,4,5,6,7,8,9,10,11,12,75\}$ \\
\hline 15 & 2 & $\{0,1,2,3,4,5,6,7,8,9,10,11,12,26,67\}$, \\
& $\{0,1,2,3,4,5,6,7,8,9,10,11,12,13,40\}$ \\
\hline 15 & 3 & $\{0,1,2,3,4,5,6,7,8,9,10,11,12,26,53\}$, \\
& $\{0,1,2,3,4,5,6,7,8,9,10,11,12,40,94\}$, \\
& $\{0,1,2,3,4,5,6,7,8,9,10,11,12,13,68\}$ \\
\hline
\end{tabular}


Table 3.6: (Initial Blocks continued)

\begin{tabular}{|l|l|l|}
\hline \hline$k$ & $c$ & Initial Blocks \\
\hline \hline 15 & 4 & $\{0,1,2,3,4,5,6,7,8,9,10,11,12,26,53\}$, \\
& & $\{0,1,2,3,4,5,6,7,8,9,10,11,12,40,135\}$, \\
& & $\{0,1,2,3,4,5,6,7,8,9,10,11,12,67,121\}$, \\
& $\{0,1,2,3,4,5,6,7,8,9,10,11,12,13,81\}$ \\
\hline 16 & 2 & $\{0,1,2,3,4,5,6,7,8,9,10,11,12,13,28,72\}$, \\
& $\{0,1,2,3,4,5,6,7,8,9,10,11,12,13,14,43\}$ \\
\hline 16 & 3 & $\{0,1,2,3,4,5,6,7,8,9,10,11,12,13,28,57\}$, \\
& & $\{0,1,2,3,4,5,6,7,8,9,10,11,12,13,43,101\}$, \\
& $\{0,1,2,3,4,5,6,7,8,9,10,11,12,13,14,73\}$ \\
\hline 17 & 2 & $\{0,1,2,3,4,5,6,7,8,9,10,11,12,13,14,30,77\}$, \\
& $\{0,1,2,3,4,5,6,7,8,9,10,11,12,13,14,15,46\}$ \\
\hline 17 & 3 & $\{0,1,2,3,4,5,6,7,8,9,10,11,12,13,14,30,61\}$, \\
& $\{0,1,2,3,4,5,6,7,8,9,10,11,12,13,14,46,108\}$, \\
& $\{0,1,2,3,4,5,6,7,8,9,10,11,12,13,14,15,78\}$ \\
\hline 18 & 2 & $\{0,1,2,3,4,5,6,7,8,9,10,11,12,13,14,15,32,82\}$, \\
& $\{0,1,2,3,4,5,6,7,8,9,10,11,12,13,14,15,16,49\}$ \\
\hline \multirow{2}{*}{3} & $\{0,1,2,3,4,5,6,7,8,9,10,11,12,13,14,15,32,65\}$, \\
& $\{0,1,2,3,4,5,6,7,8,9,10,11,12,13,14,15,49,115\}$, \\
& $\{0,1,2,3,4,5,6,7,8,9,10,11,12,13,14,15,16,83\}$ \\
\hline 19 & 2 & $\{0,1,2,3,4,5,6,7,8,9,10,11,12,13,14,15,16,34,87\}$, \\
& $\{0,1,2,3,4,5,6,7,8,9,10,11,12,13,14,15,16,17,52\}$ \\
\hline 19 & 3 & $\{0,1,2,3,4,5,6,7,8,9,10,11,12,13,14,15,16,34,69\}$, \\
& $\{0,1,2,3,4,5,6,7,8,9,10,11,12,13,14,15,16,52,122\}$, \\
& $\{0,1,2,3,4,5,6,7,8,9,10,11,12,13,14,15,16,17,88\}$ \\
\hline 20 & 2 & $\{0,1,2,3,4,5,6,7,8,9,10,11,12,13,14,15,16,17,36,92\}$, \\
& $\{0,1,2,3,4,5,6,7,8,9,10,11,12,13,14,15,16,17,18,55\}$ \\
\hline 20 & 3 & $\{0,1,2,3,4,5,6,7,8,9,10,11,12,13,14,15,16,17,36,73\}$, \\
& $\{0,1,2,3,4,5,6,7,8,9,10,11,12,13,14,15,16,17,55,129\}$, \\
& $\{0,1,2,3,4,5,6,7,8,9,10,11,12,13,14,15,16,17,18,93\}$ \\
\hline 21 & 2 & $\{0,1,2,3,4,5,6,7,8,9,10,11,12,13,14,15,16,17,18,38,97\}$, \\
& $\{0,1,2,3,4,5,6,7,8,9,10,11,12,13,14,15,16,17,18,19,58\}$ \\
\hline 21 & 3 & $\{0,1,2,3,4,5,6,7,8,9,10,11,12,13,14,15,16,17,18,38,77\}$, \\
& $\{0,1,2,3,4,5,6,7,8,9,10,11,12,13,14,15,16,17,18,58,136\}$, \\
& $\{0,1,2,3,4,5,6,7,8,9,10,11,12,13,14,15,16,17,18,19,98\}$ \\
\hline
\end{tabular}


For example, consider the initial two blocks given for the tight CD$\operatorname{CCD}(21,4,2,42)$ in Theorem 3.10. We construct the following tight CDCCD $(12,4,2,42)$ in Table 3.7. In this Table we highlight every $x \in X$ such that the pair $(0, x)$ is covered.

\begin{tabular}{llllllllllllll}
$\mathrm{B}_{0,1}$ & $\mathrm{~B}_{0,2}$ & $\mathrm{~B}_{1,1}$ & $\mathrm{~B}_{1,2}$ & $\mathrm{~B}_{2,1}$ & $\mathrm{~B}_{2,2}$ & $\mathrm{~B}_{3,1}$ & $\mathrm{~B}_{3,2}$ & $\mathrm{~B}_{4,1}$ & $\mathrm{~B}_{4,2}$ & $\mathrm{~B}_{5,1}$ & $\mathrm{~B}_{5,2}$ & $\mathrm{~B}_{6,1}$ & $\mathrm{~B}_{6,2}$ \\
0 & 0 & $5^{*}$ & $3^{*}$ & 3 & 3 & 3 & 3 & $8^{*}$ & $6^{*}$ & 6 & 6 & 6 & 6 \\
1 & 1 & 1 & 1 & $6^{*}$ & $4^{*}$ & 4 & 4 & 4 & 4 & $9^{*}$ & $7^{*}$ & 7 & 7 \\
$4^{*}$ & $2^{*}$ & 2 & 2 & 2 & 2 & $7^{*}$ & $5^{*}$ & 5 & 5 & 5 & 5 & $10^{*}$ & $8^{*}$ \\
$12^{*}$ & $7^{*}$ & $13^{*}$ & $8^{*}$ & $14^{*}$ & $9^{*}$ & $15^{*}$ & $10^{*}$ & $16^{*}$ & $11^{*}$ & $17^{*}$ & $12^{*}$ & $18^{*}$ & $13^{*}$ \\
\hline $\mathrm{B}_{7,1}$ & $\mathrm{~B}_{7,2}$ & $\mathrm{~B}_{8,1}$ & $\mathrm{~B}_{8,2}$ & $\mathrm{~B}_{9,1}$ & $\mathrm{~B}_{9,2}$ & $\mathrm{~B}_{10,1}$ & $\mathrm{~B}_{10,2}$ & $\mathrm{~B}_{11,1}$ & $\mathrm{~B}_{11,2}$ & $\mathrm{~B}_{12,1}$ & $\mathrm{~B}_{12,2}$ & $\mathrm{~B}_{13,1}$ & $\mathrm{~B}_{13,2}$ \\
$11^{*}$ & $9^{*}$ & 9 & 9 & 9 & 9 & $14^{*}$ & $12^{*}$ & 12 & 12 & 12 & 12 & $17^{*}$ & $15^{*}$ \\
7 & 7 & $12^{*}$ & $10^{*}$ & 10 & 10 & 10 & 10 & $15^{*}$ & $13^{*}$ & 13 & 13 & 13 & 13 \\
8 & 8 & 8 & 8 & $13^{*}$ & $11^{*}$ & 11 & 11 & 11 & 11 & $16^{*}$ & $14^{*}$ & 14 & 14 \\
$19^{*}$ & $14^{*}$ & $20^{*}$ & $15^{*}$ & $0^{*}$ & $16^{*}$ & $1^{*}$ & $17^{*}$ & $2^{*}$ & $18^{*}$ & $3^{*}$ & $19^{*}$ & $4^{*}$ & $20^{*}$ \\
\hline $\mathrm{B}_{14,1}$ & $\mathrm{~B}_{14,2}$ & $\mathrm{~B}_{15,1}$ & $\mathrm{~B}_{15,2}$ & $\mathrm{~B}_{16,1}$ & $\mathrm{~B}_{16,2}$ & $\mathrm{~B}_{17,1}$ & $\mathrm{~B}_{17,2}$ & $\mathrm{~B}_{18,1}$ & $\mathrm{~B}_{18,2}$ & $\mathrm{~B}_{19,1}$ & $\mathrm{~B}_{19,2}$ & $\mathrm{~B}_{20,1}$ & $\mathrm{~B}_{20,2}$ \\
15 & 15 & 15 & 15 & $20^{*}$ & $18^{*}$ & 18 & 18 & 18 & 18 & $2^{*}$ & $0^{*}$ & 0 & 0 \\
$18^{*}$ & $16^{*}$ & 16 & 16 & 16 & 16 & $0^{*}$ & $19^{*}$ & 19 & 19 & 19 & 19 & $3^{*}$ & $1^{*}$ \\
14 & 14 & $19^{*}$ & $17^{*}$ & 17 & 17 & 17 & 17 & $1^{*}$ & $20^{*}$ & 20 & 20 & 20 & 20 \\
$5^{*}$ & $0^{*}$ & $6^{*}$ & $1^{*}$ & $7^{*}$ & $2^{*}$ & $8^{*}$ & $3^{*}$ & $9^{*}$ & $4^{*}$ & $10^{*}$ & $5^{*}$ & $11^{*}$ & $6^{*}$
\end{tabular}

Table 3.7: A Tight $\operatorname{CDCCD}(21,4,2,42)$

We may also think of Theorem 3.10 in a circular manor as we did for Theorem 3.8. Consider the tight $\operatorname{CDCCD}(13,3,2,26)$. The first block is $\{0,1,4\}$ and the second block is $\{0,2,7\}$. These blocks are highlighted in blue and pink respectively in Figure 3.3 and the unchanged subset is highlighted black. 


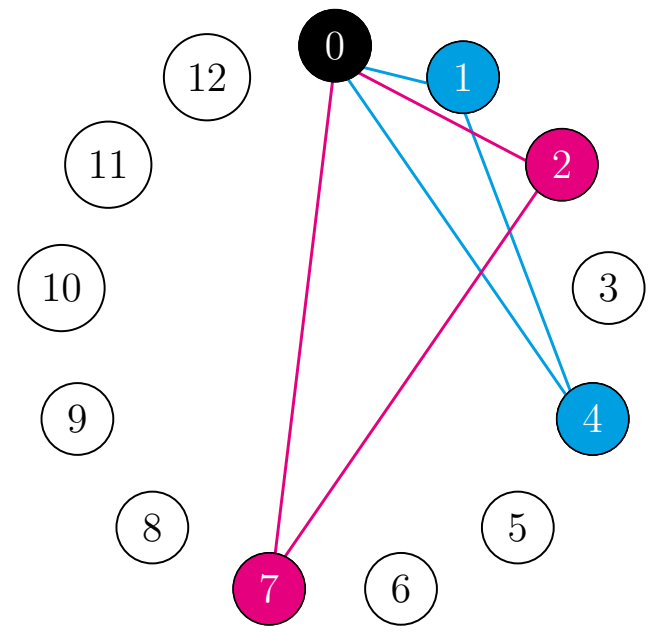

Figure 3.3: $c=2, k=3$ tight $\operatorname{CDCCD}(13,3,2,26)$

We alternate rotating the colours by 1 so that the odd blocks are blue and the even pink. Note that taking every difference in $\mathrm{B}_{1}$ and $\mathrm{B}_{2}$ involving at least one introduced point produces the differences $1,2, \ldots, 12(\bmod 13)$ and so these differences are in every pair of consecutive blocks. This produces the tight CDCCD $(13,3,2,26)$ as seen in Table 3.8 .

\begin{tabular}{lllllllllllllllll}
$\mathrm{B}_{1}$ & $\mathrm{~B}_{2}$ & $\mathrm{~B}_{3}$ & $\mathrm{~B}_{4}$ & $\mathrm{~B}_{5}$ & $\mathrm{~B}_{6}$ & $\mathrm{~B}_{7}$ & $\mathrm{~B}_{8}$ & $\mathrm{~B}_{9}$ & $\mathrm{~B}_{10}$ & $\mathrm{~B}_{11}$ & $\mathrm{~B}_{12}$ & $\mathrm{~B}_{13}$ & $\mathrm{~B}_{14}$ & $\mathrm{~B}_{15}$ & $\mathrm{~B}_{16}$ & $\mathrm{~B}_{17}$ \\
$0^{*}$ & 0 & $1^{*}$ & 1 & $2^{*}$ & 2 & $3^{*}$ & 3 & $4^{*}$ & 4 & $5^{*}$ & 5 & $6^{*}$ & 6 & $7^{*}$ & 7 & $8^{*}$ \\
1 & $2^{*}$ & 2 & $3^{*}$ & 3 & $4^{*}$ & 4 & $5^{*}$ & 5 & $6^{*}$ & 6 & $7^{*}$ & 7 & $8^{*}$ & 8 & $9^{*}$ & 9 \\
$4^{*}$ & $7^{*}$ & $5^{*}$ & $8^{*}$ & $6^{*}$ & $9^{*}$ & $7^{*}$ & $10^{*}$ & $8^{*}$ & $11^{*}$ & $9^{*}$ & $12^{*}$ & $10^{*}$ & $0^{*}$ & $11^{*}$ & $1^{*}$ & $12^{*}$ \\
\hline $\mathrm{B}_{18}$ & $\mathrm{~B}_{19}$ & $\mathrm{~B}_{20}$ & $\mathrm{~B}_{21}$ & $\mathrm{~B}_{22}$ & $\mathrm{~B}_{23}$ & $\mathrm{~B}_{24}$ & $\mathrm{~B}_{25}$ & $\mathrm{~B}_{26}$ & & & & & & & \\
8 & $9^{*}$ & 9 & $10^{*}$ & $10^{*}$ & $11^{*}$ & 11 & $12^{*}$ & 12 & & & & & & & \\
$10^{*}$ & 10 & $11^{*}$ & 11 & $12^{*}$ & 12 & $0^{*}$ & 0 & $1^{*}$ & & & & & & & \\
$2^{*}$ & 0 & 3 & 1 & 4 & 2 & 5 & 3 & 6 & & & & & & & &
\end{tabular}

Table 3.8: Tight CDCCD $(13,3,2,26)$ 


\section{Chapter 4}

\section{Conclusion}

In this paper we reviewed the literature on circular and non-circular SCCD. We develop a small recursion for all circular and non-circular $\operatorname{SCCD}(v, 3,2, b)$ which provides an alternative proof of existence. We integrated search techniques from exhaustive searches for tight SCCD and tight CSCCD to implement an efficient search for CSCCDs. With this search we generate all 313 distinct $\operatorname{CSCCD}(12,4,2,22)$. We began a preliminary examination for higher strength SCCD and CSCCD.

In Chapter 3 we explore DCCD and CDCCD. Our examination produced a theorem to increase $v$ in CDCCD and DCCD analogous to the $\operatorname{SCCD}(v+1, k, 2, b)$ construction. We also completely solved the existence problem for tight $\operatorname{CDCCD}(v, 3,2, b)$ and tight $\operatorname{DCCD}(v, 3,2, b)$ and produced some families of designs for some given $k$. We solve CDCCD $(4 k-2,2 k, 2,2 k-$ $1), \operatorname{CDCCD}(4 k-1,2 k+1,2,2 k-1), \operatorname{CDCCD}(4 k-5, k, 2,4 k-5)$, and $\operatorname{CDCCD}\left(c(4 k-6)+1, k, 2, c^{2}(4 k-6)+c\right)$ for specific values of $c, k$.

We believe that the most significant contributions to the field involve the examination of double change covering designs. This is because we completely solve the existence problem for tight $k=3, s=2$ as well as provide some insight into more possible single change constructions using the difference methods technique explored. We also think that the strength $3, v+1$ recursion with the expansion set and the $v+v^{\prime}$ recursions are significant. 


\subsection{Looking forward}

We would like to continue the examination of SF-arrays for strength 3 CSCCD. Once we know the restrictions on the skeletons of singletons and pairs, we can use both to significantly reduce the work of a recursive search. This search will provide us with small designs we can use as ingredients for some recursive constructions on $v$.

We would like to re-visit the recursive search for CSCCD and parallelize it to find CSCCD with larger $k$. Furthermore, we would like to examine how we may construct SF-arrays for economical CSCCD with strength 2 in order to make this recursion more dynamic.

We want to consider the use of SCCD, CSCCD, DCCD, and CDCCD for use in cache-aware programming. Change designs suite situations where operations or tests are inexpensive compared to the cost of interchanging elements. Updating the cache is time expensive compared to reading as it requires reading RAM which is an order of magnitudes slower, so algorithms which utilized double and single change designs to manage cache content could offer significant time savings.

We believe that we can find the following family of circular DCCD.

Conjecture 4.1. All circular DCCD $\left(c(4 k-6)+1, k, 2, c^{2}(4 k-6)+c\right), c \geq 1$ exist.

By Theorem 3.8 we know that this is true when $c=1$. We have code that will run for small values of $c$ and $v$ which produces the base blocks for such designs. We am currently using the idea related to Skolem sequences to examine these cases when $k=4$.

Furthermore, we ask whether we may use a set of initial blocks as in Conjecture 4.1 to produce initial blocks for families of CSCCD.

We would like to get a general construction for economic $\operatorname{CDCCD}(v, 3,2, b)$.

We would like to generalize Theorem 2.1 for values of $k>3$. In particular we would like to start by examining potential constructions for $\operatorname{SCCD}(v, 4,2, b)$ by examining all possible $\operatorname{SCCD}(12,4,2,22)$.

Suppose $c: X \rightarrow \mathbb{R}$ is a cost function and $\left(X, \mathcal{L}=\left(B_{i}\right)_{i=1}^{b}\right)$ is a m-CCD. The cost of $(X, \mathcal{L})$ is

$$
\sum_{i=1}^{b} \sum_{x \in B_{i+1} \backslash B_{i}} c(x)
$$


If $c(x)=c \forall x$ then a minimal $\mathrm{m}$-CCD minimizes the cost. Suppose that $c: X \rightarrow \mathbb{R}$ is is not a constant function, can we develop methods to construct minimal $\operatorname{SCCD}(v, k, s, b)$ ?

Can we use SCCD or DCCD to formulate a logic puzzle with a unique solution? If so what would the minimum amount of information required be to solve the puzzle?

Let $\left(\begin{array}{l}X \\ s\end{array}\right)$ denote the set of all $s$-subsets of $X$. A disjoint single change covering design, $\operatorname{DSCCD}\left(v, v^{\prime}, k, s, b\right),\left(X, X^{\prime}, \mathcal{L}\right)$, is a $v$-set $X$, a $v^{\prime}$-set $X^{\prime}$ and a single change list of blocks $\mathcal{L}=\left(B_{i}\right)_{i=1}^{b}$ of size $k$ where every $s$-set in $\left(\begin{array}{c}X \\ s\end{array}\right) \dot{\cup}\left(\begin{array}{c}X^{\prime} \\ s\end{array}\right)$ must occur on at least one block. What is minimum $b$ for $v, v^{\prime}, k, s$ ?

Let $H \subseteq X$ and $\mathcal{L}=\left(B_{i}\right)_{i=1}^{b}$, what is the minimum $b$ required to cover all the pairs $\left(\begin{array}{c}X \\ 2\end{array}\right) \backslash\left(\begin{array}{c}H \\ 2\end{array}\right)[18]$.

We end with a speculative idea. Suppose we had a group of $v$ people and restricted contact in effect in society. Suppose that $k n$ of them meet simultaneously in $n$ groups of $k$ people. Suppose that only one person can leave and enter a meeting at a time. After leaving these meetings each of the $n$ people must isolate for $t$ days to monitor for possible infections. For example, consider Table 4.1.

\begin{tabular}{lllll}
\multicolumn{5}{c}{ Group 1} \\
$\mathrm{~B}_{1}^{1}$ & $\mathrm{~B}_{2}^{1}$ & $\mathrm{~B}_{3}^{1}$ & $\mathrm{~B}_{4}^{1}$ & $\ldots$ \\
$1^{*}$ & $4^{*}$ & 4 & 4 & \\
2 & 2 & $5^{*}$ & 5 & \\
3 & 3 & 3 & $6^{*}$ & \\
\multicolumn{5}{c}{} \\
\multicolumn{5}{c}{ Group $n$} \\
$\mathrm{~B}_{1}^{n}$ & $\mathrm{~B}_{2}^{n}$ & $\mathrm{~B}_{3}^{n}$ & $\mathrm{~B}_{4}^{n}$ & $\ldots$ \\
$\mathrm{v}^{*}$ & $\mathrm{v}-3^{*}$ & $\mathrm{v}-3$ & $\mathrm{v}-3$ & \\
$\mathrm{v}-1$ & $\mathrm{v}-1$ & $\mathrm{v}-4^{*}$ & $1^{*}$ & \\
$\mathrm{v}-2$ & $\mathrm{v}-2$ & $\mathrm{v}-2$ & $\mathrm{v}-2$ &
\end{tabular}

Table 4.1: Partial example of $k n$ people in $n$ groups of $k, t=2$

Let a single change covering family, $\operatorname{SCCF}(v, k, n, t, s, b)$, be a set of $n$ single change lists of blocks $\left(B_{i}^{j}\right)_{i=1}^{b}$ for $1 \leq j \leq n$ and if $x \in B_{i}^{j}$ then $x \notin B_{l}^{j}, \forall i<l \leq i+t$ What is the minimum $b$ for a $\operatorname{SCCF}(v, k, n, t, s, b)$. Can minimal, economical or tight SCCF be constructed?

Suppose further the possibility that blocks vary in size. Let a single 
change covering family, $\operatorname{SCCF}\left(v, k_{1}, \ldots, k_{r}, n, t, s, b\right)$ be a set of $n$ single change lists of blocks with the same isolation time constraints. For what $b$ do $\operatorname{SCCF}\left(v, k_{1}, \ldots, k_{r}, n, t, s, b\right)$ exist? For what $v, k_{1}, \ldots, k_{r}, n, t, s$ can tight, economical or minimal SCCF be constructed? 


\section{Glossary}

1-factor A perfect matching or 1-factor of a graph $G$ is a subset of edges $M$ such that every vertex $v \in V$ is adjacent to exactly one edge $e \in M .2$

1-factorization A partition of the edges of a graph into perfect matchings is a 1 -factorization. 2

balanced incomplete block designs A balanced incomplete block design $((v, k, \lambda)$-BIBD $)$, is a set of $k$ blocks from a $v$-set $X$ where each pair from $X$ occurs in exactly $\lambda$ blocks. 3

circular The property that the number of changes required between consecutive blocks holds between $\mathrm{B}_{1}$ and $\mathrm{B}_{b} .6,39$

clout A clout is a set of elements less then the strength of a design that we consider for the sake of SF-arrays of higher strength designs. 34

covered A $s$-set, $S$, is covered on $\mathrm{B}_{i}$ if at least one $x \in S$ is introduced in $\mathrm{B}_{i} .6,39$

cycle A cycle in a graph $G$ is a path in $G$ where the first and last vertex are joined by an edge. 2

difference family $\mathrm{A}(v, k, \lambda)$-difference family is a collection $\left\{D_{1}, \ldots, D_{t}\right\}$ of $k$-subsets of $G,|G|=v$, where $\partial D_{1} \cup \ldots \cup \partial D_{t}=\lambda G \backslash\{0\} .3$

difference set A difference family with $t=1$ is a $(v, k, \lambda)$-difference set. 3

disjoint-capable An expansion set is disjoint-capable if it satisfies conditions that utilize the unchanged subsets. 19 
double change covering design A double change covering design $(\operatorname{DCCD}(v, k, s, b))$ is a $v$ set and an ordered list of blocks of size $k$ where every $s$-set must occur on at least one block. Every block differs from the next by exactly two elements. 40

economical A change design is economical if the lower bound on the minimum required blocks is met. 8,40

end permutation An end permutation is an SCCD obtained by swapping two points in all blocks after the $i^{\text {th }}$ block. 13

equivalent Two designs are equivalent if one may be obtained by permuting the elements or cyclically shifting the blocks of the other. 28

expansion set A partition of $V$ using unchanged subsets. 7, 36

finishes An element finishes in a block of an SF-array if it is only introduced once and is removed in the next block. 16, 35

front loading Front loading the design is when we cycle the SF-skeleton so that the first block will contain as many elements introduced exactly once as possible. 28

graph A graph is an ordered pair $G=(V, E)$ of vertices, $V$, and edges, $E$, such that each edge consists of two end vertices. 2

Hamilton cycle A Hamilton cycle in a graph $G$ is a cycle visiting all the vertices $V$ of $G$ only once. 2

Hamilton path A Hamilton path in a graph $G$ is a path visiting all the vertices $V$ of $G$ only once. 2

hole A hole is the pair of elements missing from a single change covering design with a hole. 10

inner expansion set An expansion set that does not use $U_{0}$ or $U_{b}$. 7, 36, 39

introduced An element $x$ is introduced in a block $B_{i}$ if $x \notin B_{i-1}$. In a non-circular SCCD every element in $B_{1}$ is introduced. 6, 35, 39 
maximum introduction limit The most number of introductions any element may be introduced in a single design. 15

minimal A change design is minimal if it uses the fewest blocks possible to complete the change design. 6, 39

minor variant A minor variant is an SCCD obtained by swapping two consecutive blocks where this preserves the single change property. 12

multi-change covering design A multi-change covering design (m-CCD $(v, k, s, b)$ ) is a $v$ set and an ordered list of blocks of size $k$ where every $s$-set is must occur on at least one block. Every block differs from the next by exactly $m$ elements. 39

outer expansion set An expansion set that uses $U_{0}, U_{b}$ or both. 7, 36, 39

path A path in a graph $G$ is a walk with distinct vertices. 2

perfect matching See 1-factor. 2

persistence condition Conditions that dictate if an element must be in the unchanged subset based on the number of remaining pairs to be covered. 29

removal conditions Conditions that dictate if an element may be removed in the next block based on the number of remaining pairs to be covered. 13

removed An element $x$ is removed in block $B_{i}$ if $x \in B_{i-1}$ and $x \notin B_{i}$. 6 , 39

run A group of successive blocks containing $x .16,35$

SCCD A single change covering design $(\operatorname{SCCD}(v, k, s, b))$ is a sequence of $b k$-sets, called blocks, of a $V$-set in which exactly one element differs between consecutive blocks and every $s$-set of $V$ is in some block. 1, 5

SF-array The SF-array of $x$ in a design is a list describing the introductions and removals of all elements introduced exactly once over a run of $x$ where $x$ is introduced once. 16 
SF-skeleton A SF-skeleton is an expanded SF-array that may be used to partially fill in the design. 17

single change covering design with a hole A single change covering design with a hole, SCCDH is a SCCD such that every pair of elements of $V$ occur on at least one block except for one pair, $\{\mathrm{x}, \mathrm{y}\}$, the hole, where one of $x$ or $y$ appear in $B_{1}$ or $B_{b} .10$

starts An elements starts in a block of an SF-array if it is only introduced once and is introduced on the current block. 16, 35

Steiner system A Steiner system $\mathrm{S}(t, k, v), 2 \leq t \leq k \leq v$, is a $v$-set $V$ with a family $\mathcal{B}$ of $k$-subsets of $V$ (blocks), such that every $t$-subset of $V$ is contained in exactly one block, $t, k, v \in \mathbb{Z}^{+} .2$

Steiner Triple System A Steiner Triple System, $\operatorname{STS}(v)$, is a $\mathrm{S}(2,3, v) .3$

strength The strength of a design is the size of the set $S$ that we must cover in at least one block of the design. 35

tight A change design is tight if it is economical and the bound on minimum blocks is met with equality. 8,40

tight block A block is tight if it does not cover any $s$-set covered in a different block. 11

unchanged subset The unchanged subset is $U_{i}=B_{i} \cap B_{i+1}$, the elements that do not change between consecutive blocks. $6,36,39$

V-expansion set A $V$-expansion set is an expansion set. 36

W-expansion set A $W$-expansion set is a partition of $W \subset V$ using the expansion set. 36,39

walk $\mathrm{A}$ walk in a graph $G$ is a sequence $W=v_{0} e_{1} v_{1} \ldots v_{l-1} e_{l} v_{l}$ where $v$ and $e$ are alternating vertices and edges of $G$ such that $v_{i-1}$ and $v_{i}$ are the ends of edge $e_{i}, 1 \leq i \leq l .2$

weight The weight of a block is a value based on the number of ' $\mathrm{S}$ ' and ' $\mathrm{F}$ ' in the SF-array. 16 


\section{Bibliography}

[1] J. Akiyama and M. Kano. Factors and Factorizations of Graphs. Springer, 2011.

[2] J.F. Meyer B.E. Aupperle. "Fault-Tolerant BIBD Networks". In: The Eighteenth International Symposium on Fault-Tolerant Computing. IEEE, 1988, pp. 306-311.

[3] A. L. Chafee. Recursively Constructing Tight and Economical SingleChange Covering Designs and Circular Single-Change Covering Designs (Some New $(v, 4)$ and $(v, 5)$ Designs). Carleton University Undergraduate Thesis. Aug. 2018.

[4] C.J. Colbourn and J.H. Dinitz. Handbook of Combinatorial Designs. Second Edition. Champman and Hall/CRC, 2007.

[5] R.A. Fisher. The Design of Experiments. Macmillan, 1935.

[6] J. C. Gower and D. A. Preece. "Generating successive incomplete blocks with each pair of elements in at least one block". In: Comin. Theory 12 (1972), pp. 81-97.

[7] P. Horak and A. Rosa. "Decomposing Steiner Triple Systems into Small Configurations". In: Ars Combinatoria 26 (Dec. 1988), pp. 91-105.

[8] J. P. McSorley. "Single-change circular covering designs". In: vol. 197/198. 16th British Combinatorial Conference (London, 1997). 1999, pp. 561588.

[9] J.A. Nelder. "The efficient formation of a triangular array with restricted storage for data". In: Applied Statistics 18 (1969), pp. 203206.

[10] N.C.K Phillips. "Finding tight single-change covering designs with $v=$ 20, $k=5$ ". In: vol. 231. 1-3. 17th British Combinatorial Conference (Canterbury, 1999). 2001, pp. 403-409. 
[11] N.C.K. Phillips and D.A. Preece. "Tight single-change covering designs with $v=12, k=4$ ". In: vol. 197/198. 16th British Combinatorial Conference (London, 1997). 1999, pp. 657-670.

[12] D. A. Preece et al. "Tight single-change covering designs". In: Utilitas Math. 47 (1995), pp. 55-84. ISSN: 0315-3681.

[13] D. R. Stinson. Combinatorial designs. Constructions and analysis, With a foreword by Charles J. Colbourn. Springer-Verlag, New York, 2004, pp. xvi+300. ISBN: 0-387-95487-2.

[14] G. H. J. Van Rees. "Single-change covering designs. II". In: vol. 92. Twenty-second Manitoba Conference on Numerical Mathematics and Computing (Winnipeg, MB, 1992). 1993, pp. 29-32.

[15] W. D. Wallis, J. L. Yucas, and G.-H. Zhang. "Single change covering designs". In: Des. Codes Cryptogr. 3.1 (1993), pp. 9-19. ISSN: 09251022 .

[16] D. B. West. Introduction to Graph Theory. Second Edition. Upper Saddle River, NJ 07458: Prentice Hall, 2001.

[17] H. Huang Y. Chen and S. Wang. "Video Scrambling and Fingerprinting for Digital Right Protection". In: 2012 International Symposium on Computer, Consumer and Control, Taichung. IEEE, 2012, pp. 471474.

[18] Guo-Hui Zhang. "Some new bounds of single-change covering designs". In: SIAM J. Discrete Math. 7.2 (1994), pp. 166-171. ISSN: 0895-4801. 